This paper was withdrawn on April 22, 2011 (ACS Catal. 2011, 1, 573).

\title{
Enantioselective Synthesis of Chromans with a Quaternary Stereogenic Centre through Catalytic Asymmetric Cascade Reactions
}

Qiu-Lin Hua, Chao Li, Xu-Fan Wang, Liang-Qiu Lu, Jia-Rong Chen* and Wen-Jing Xiao*

The Key Laboratory of Pesticide and Chemical Biology, Ministry of Education, College of Chemistry, Central China Normal University, 152 Luoyu Road, Wuhan, Hubei 430079, China

Fax: (+86)-27-67862041; E-mail: wxiao@mail.ccnu.edu.cn; jiarongchen2003@yahoo.com.cn

\section{Supporting Information}

\section{Contents}

1. General Methods $\quad$ S-1

2. Materials $\quad$ S-1

3. Reaction Optimisation $\quad \mathrm{S}-2$

4. General Procedure and Spectral Data of Products $\quad$ S-6

5. Synthesis transformation of the cascade reaction adduct 3a $\quad$ S-11

6. Copies of ${ }^{1} \mathrm{H}$ NMR and ${ }^{13} \mathrm{C}$ NMR Spectrums $\quad \mathrm{S}-12$

$\begin{array}{ll}\text { 7. The copy of HPLC chromatograms } & \text { S-30 }\end{array}$ 


\section{General methods}

Unless otherwise noted, materials were purchased from commercial suppliers and used without further purification. All the solvents were treated according to general methods. Flash column chromatography was performed using 200-300 mesh silica gel. ${ }^{1} \mathrm{H}$ NMR spectra were recorded on Varian Mercury 400/600 (400/600 MHz) spectrophotometers. Chemical shifts $(\delta)$ are reported in ppm from the solvent resonance as the internal standard $\left(\mathrm{CDCl}_{3}: 7.26 \mathrm{ppm}\right)$. Data are reported as follows: chemical shift, multiplicity $(\mathrm{s}=$ singlet, $\mathrm{d}=$ doublet, $\mathrm{t}=$ triplet, $\mathrm{dd}=$ doublet of doublets, $\mathrm{m}$ $=$ multiplet), coupling constants $(\mathrm{Hz})$ and integration. ${ }^{13} \mathrm{C}$ NMR spectra were recorded on Varian Mercury 400/600 (100/150 MHz) with complete proton decoupling spectrophotometers $\left(\mathrm{CDCl}_{3}\right.$ : 77.0 ppm). Mass spectra were measured on API 2000 LC/MS/MS (ESI-MS) or Waters Synapt G2 (MALDI). Enantiomeric ratios were determined by chiral HPLC on Agilent 1100 series with chiral columns (chiralpak AS-H column, chiralpak AD-H column) with hexane and 2-propanol as solvents. Optical rotations were measured with JASCO P-1020 polarimeter.

\section{Materials}

All substrates were prepared according to literature procedures ${ }^{1}$ and characterizations of nitroolefin enoates were in accordance with previously reported data.

\section{Reference}

1 (a) Ciganek, E. Synthesis 1995, 1311-1314. (b) Denmark, S. E.; Senanayaket, C. B. W. J. Org. Chem. 1993, 58, 1853-1858. (c) Martin, N. J. A.; Cheng, X.; List, B. J. Am. Chem. Soc., 2008, 130, 13862-13863; (d) Lu, L.-Q.; Li, F.; An, J.; Zhang, J.-J.; An, X.-L.; Hua, Q.-L.; Xiao, W.-J. Angew. Chem. Int. Ed., 2009, 48, 9542-9545. (e) Wang, X.-F.; Hua, Q.-L.; Cheng, Y.; An, X.-L.; Yang, Q.-Q.; Chen, J.-R.; Xiao, W.-J. Angew. Chem., Int. Ed., 2010, 49, 8379-8383. 


\section{Reaction Optimisation}

Table 1s. The screening of solvent for the cascade aza-Michael/Michael addition reaction. ${ }^{[a]}$

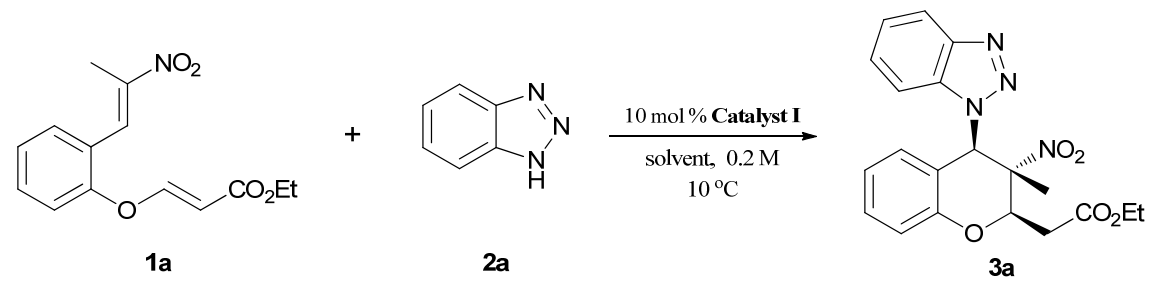

\begin{tabular}{|c|c|c|c|c|c|c|}
\hline Entry & Solvents & $t(d)$ & Product & Yield[\% []$^{[b]}$ & ee $(\%)^{[c]}$ & $\mathrm{dr}^{[\mathrm{c}]}$ \\
\hline 1 & $\mathrm{CH}_{2} \mathrm{Cl}_{2}$ & 5 & $3 \mathbf{a}$ & 90 & 87 & $81: 19$ \\
\hline 2 & $\mathrm{CHCl}_{3}$ & 5 & $3 \mathbf{a}$ & 52 & 73 & $75: 25$ \\
\hline 3 & $\mathrm{ClCH}_{2} \mathrm{CH}_{2} \mathrm{Cl}$ & 6 & $3 a$ & 81 & 85 & $80: 20$ \\
\hline 4 & THF & 6 & $3 a$ & 32 & 71 & $78: 22$ \\
\hline 5 & $\mathrm{Et}_{2} \mathrm{O}$ & 6 & $3 \mathbf{a}$ & 70 & 77 & $84: 16$ \\
\hline 6 & $\mathrm{CH}_{3} \mathrm{CN}$ & 5 & $3 a$ & 96 & 73 & $85: 15$ \\
\hline 7 & Toluene & 5 & $3 a$ & 78 & 67 & $76: 24$ \\
\hline 8 & xylenes & 5 & $3 a$ & 70 & 65 & $75: 25$ \\
\hline 9 & $\mathrm{CH}_{3} \mathrm{OH}$ & 7 & $3 a$ & 75 & 13 & $83: 17$ \\
\hline 10 & $\mathrm{CH}_{3} \mathrm{CH}_{2} \mathrm{OH}$ & 7 & $3 a$ & 82 & 32 & $73: 27$ \\
\hline 11 & Hexane & 7 & $3 a$ & 72 & 73 & $77: 23$ \\
\hline 12 & ${ }^{t} \mathrm{BuOMe}$ & 7 & $3 a$ & 87 & 81 & $83: 17$ \\
\hline 13 & $\mathrm{DMF}$ & 7 & $3 a$ & 72 & 6 & $93: 7$ \\
\hline
\end{tabular}

[a] Reactions were carried out with $\mathbf{1 a}(0.40 \mathrm{mmol}), \mathbf{2 a}(0.60 \mathrm{mmol})$, Catalyst $\mathbf{I}(0.04 \mathrm{mmol})$ in solvent $(2.0 \mathrm{ml})$ at $10^{\circ} \mathrm{C}$. [b] Yield of isolated product. [c] Determined by chiral HPLC.

Table 2s. The screening of temperature for the cascade aza-Michael/Michael addition reaction. ${ }^{\text {[a] }}$<smiles>CCOC(=O)C=COc1ccccc1C=C(C)[N+](=O)[O-]</smiles>

$1 \mathbf{a}$

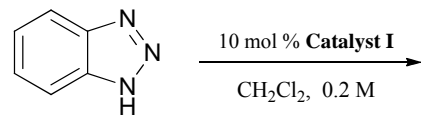

$2 a$<smiles>CCOC(=O)C[C@H]1Oc2ccccc2[C@@H](n2nnc3ccccc32)[C@@]1(C)[N+](=O)[O-]</smiles>

3a 


\begin{tabular}{ccccccc}
\hline Entry & Temperature $\left({ }^{\circ} \mathrm{C}\right)$ & $\mathrm{t}(\mathrm{d})$ & Product & Yield $[\%]^{[\mathrm{b}]}$ & $\mathrm{ee}(\%)^{[\mathrm{c}]}$ & $\mathrm{dr}{ }^{[\mathrm{c}]}$ \\
\hline 1 & 0 & 12 & $\mathbf{3 a}$ & 71 & 86 & $82: 18$ \\
$\mathbf{2}$ & $\mathbf{1 0}$ & $\mathbf{5}$ & $\mathbf{3 a}$ & $\mathbf{9 0}$ & $\mathbf{8 7}$ & $\mathbf{8 1 : 1 9}$ \\
\hline 3 & 25 & 5 & $\mathbf{3 a}$ & 57 & 80 & $80: 20$ \\
\hline
\end{tabular}

[a] Reactions were carried out with $1 \mathbf{a}(0.40 \mathrm{mmol}), 2 \mathbf{a}(0.60 \mathrm{mmol})$, Catalyst $\mathbf{I}(0.04 \mathrm{mmol})$ in $\mathrm{CH}_{2} \mathrm{Cl}_{2}(2.0 \mathrm{ml})$ at $\mathrm{x}{ }^{\circ} \mathrm{C}$. [b] Yield of isolated product. [c] Determined by chiral HPLC.

Table 3s. The screening of bifunctional catalyst for the cascade aza-Michael/Michael addition reaction. ${ }^{[\mathrm{a}]}$
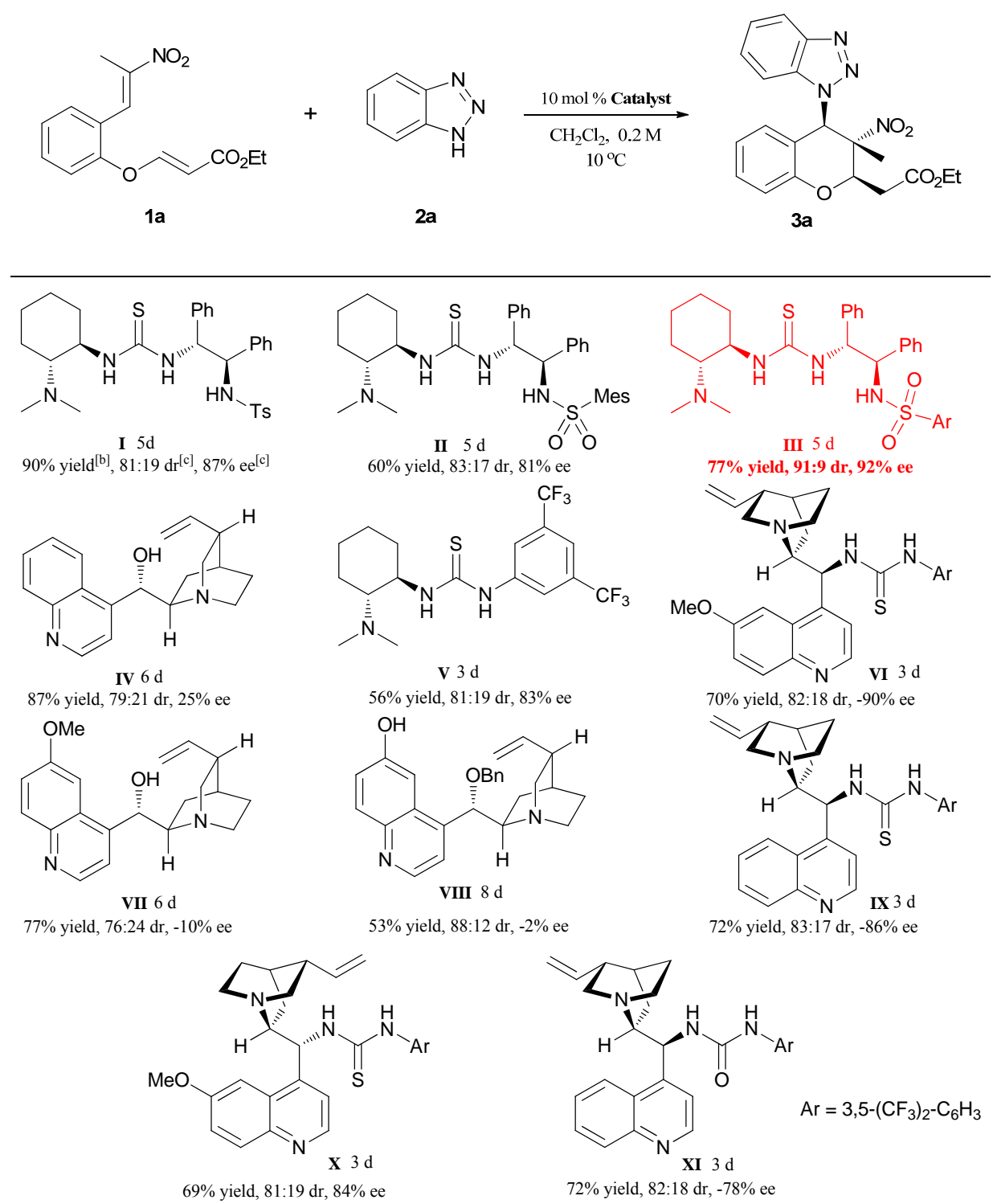

[a] Reactions were carried out with 1a $(0.40 \mathrm{mmol})$, 2a $(0.60 \mathrm{mmol})$, Catalyst $(0.04 \mathrm{mmol})$ in $\mathrm{CH}_{2} \mathrm{Cl}_{2}(2.0 \mathrm{ml})$ at $10^{\circ} \mathrm{C}$. [b] Yield of isolated product. [c] Determined by chiral HPLC. 
Table 4s. The screening of equivalent of benzotriazole 2a for the cascade aza-Michael/Michael addition reaction. ${ }^{[\mathrm{a}]}$

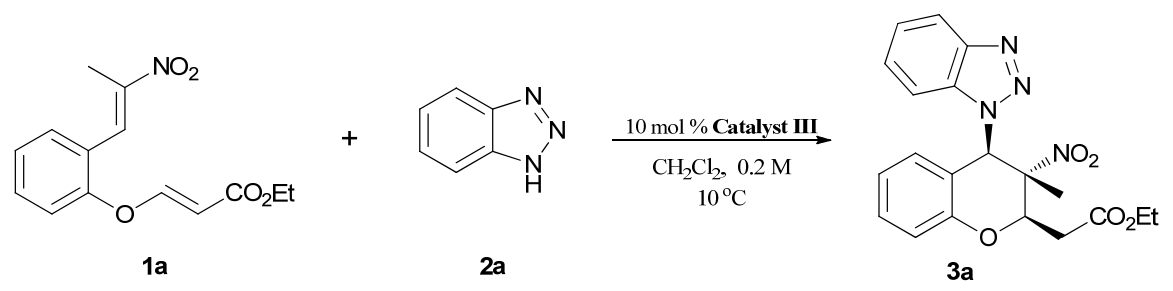

\begin{tabular}{ccccccc}
\hline Entry & Equivalent of 2a & $\mathrm{t}(\mathrm{d})$ & Product & Yield $[\%]^{[\mathrm{b}]}$ & ee $(\%)^{[\mathrm{c}]}$ & $\mathrm{dr}^{[\mathrm{c}]}$ \\
\hline $\mathbf{1}$ & $\mathbf{1 . 5}$ & $\mathbf{5}$ & $\mathbf{3 a}$ & $\mathbf{7 7}$ & $\mathbf{9 2}$ & $\mathbf{9 1 : 9}$ \\
\hline 2 & 2.0 & 5 & $\mathbf{3 a}$ & 78 & 87 & $86: 14$ \\
3 & 3.0 & 5 & $\mathbf{3 a}$ & 83 & 81 & $83: 17$ \\
\hline
\end{tabular}

[a] Reactions were carried out with 1a $(0.40 \mathrm{mmol})$, 2a, Catalyst III $(0.04 \mathrm{mmol})$ in $\mathrm{CH}_{2} \mathrm{Cl}_{2}$ $(2.0 \mathrm{ml})$ at $10^{\circ} \mathrm{C}$. [b] Yield of isolated product. [c] Determined by chiral HPLC.

\section{General Procedure and Spectral Data of Products}

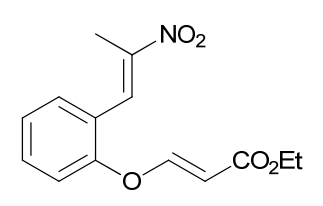

$1 \mathrm{a}$

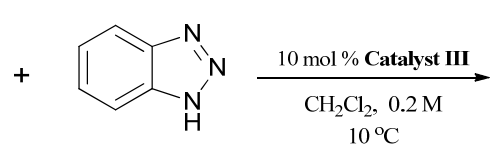

$2 a$

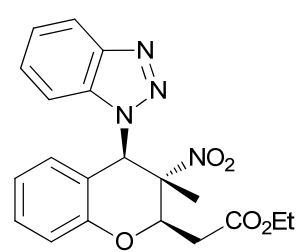

$3 \mathbf{a}$

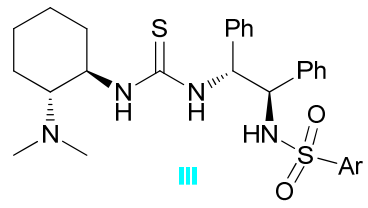

$\mathrm{Ar}=3,5-\left(\mathrm{CF}_{3}\right)_{2}-\mathrm{Ph}$

\section{General procedure for the asymmetric cascade aza-Michael/Michael addition reaction.}

A mixture of nitroolefin enoate $1 \mathrm{a}(0.40 \mathrm{mmol})$ and catalyst $\mathbf{I I I}(0.040 \mathrm{mmol})$ in $\mathrm{CH}_{2} \mathrm{Cl}_{2}(2.0 \mathrm{~mL})$ was stirred at $10{ }^{\circ} \mathrm{C}$ for $15 \mathrm{~min}$, then benzotriazole $2 \mathrm{a}(0.60 \mathrm{mmol})$ was added to the reaction mixture. Upon completion (monitored by TLC analysis), the mixture was purified by flash column chromatography on silica gel (petroleum ether/ethyl acetate $=7 / 1$ to $5 / 1$ as eluants) to afford the desired product 3a.

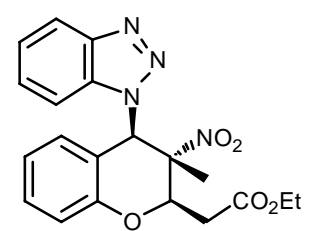

Ethyl 2-((2R,3S,4R)-4-(1H-benzo[d][1,2,3]triazol-1-yl)-3-methyl-3-nitrochr oman-2-yl)acetate (3a). Prepared according to the general procedure from 1a $(0.40 \mathrm{mmol})$, 2a $(0.60 \mathrm{mmol})$, catalyst III $(0.040 \mathrm{mmol})$ and $\mathrm{CH}_{2} \mathrm{Cl}_{2}(2.0 \mathrm{~mL})$ at $10{ }^{\circ} \mathrm{C}$ for $5 \mathrm{~d}$ to provide the title compound as a white solid (77\% yield, $92 \%$ ee, $91: 9 \mathrm{dr}) .{ }^{1} \mathbf{H}$ NMR $\left(600 \mathrm{MHz}, \mathrm{CDCl}_{3}\right) \delta(\mathrm{ppm}) 8.12(\mathrm{~d}, J=8.5 \mathrm{~Hz}, 1 \mathrm{H}), 7.43-$ $7.39(\mathrm{~m}, 2 \mathrm{H}), 7.35-7.32(\mathrm{~m}, 2 \mathrm{H}), 7.07-7.06(\mathrm{~m}, 1 \mathrm{H}), 6.95-6.92(\mathrm{~m}, 1 \mathrm{H}), 6.56(\mathrm{~s}, 1 \mathrm{H}), 5.31(\mathrm{~d}, J$ $=9.7 \mathrm{~Hz}, 1 \mathrm{H}), 4.28-4.24(\mathrm{~m}, 2 \mathrm{H}), 2.84(\mathrm{dd}, J=16.0,9.8 \mathrm{~Hz}, 1 \mathrm{H}), 2.42(\mathrm{~d}, J=16.4 \mathrm{~Hz}, 1 \mathrm{H}), 1.54$ (s, 3H), 1.32 (t, $J=7.1 \mathrm{~Hz}, 3 \mathrm{H}) ; .{ }^{13} \mathbf{C}$ NMR (150 MHz, $\left.\mathrm{CDCl}_{3}\right) \delta(\mathrm{ppm}) 168.58,152.83,145.55$, 
130.33, 127.98, 124.30, 122.52, 120.25, 117.31, 88.75, 76.29, 63.05, 61.26, 34.56, 13.97, 12.23; HRMS (ESI): Calcd for $\mathrm{C}_{20} \mathrm{H}_{20} \mathrm{~N}_{4} \mathrm{O}_{5}[\mathrm{M}+\mathrm{Na}]$ : 419.1331. Found: 419.1315; $[\alpha]_{\mathrm{D}}{ }^{29}=43.20(\mathrm{c}=1.04$, $\mathrm{CHCl}_{3}$ ); the enantiomeric excess was determined by chiral HPLC (Chiralpak AS column: hexane $/ 2$-propanol $=60 / 40,0.5 \mathrm{~mL} / \mathrm{min}, 254 \mathrm{~nm}, \mathrm{t}_{\text {major }}=43.03 \mathrm{~min}, \mathrm{t}_{\operatorname{minor}}=24.53 \mathrm{~min}$ ).

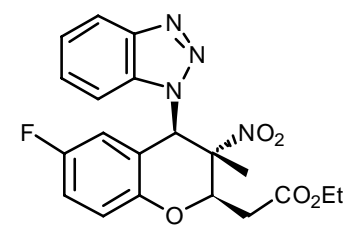

Ethyl 2-((2R,3S,4R)-4-(1H-benzo[d][1,2,3]triazol-1-yl)-6-fluoro-3-methyl3-nitrochroman-2-yl)acetate (3b). Prepared according to the general procedure from $\mathbf{1 b}(0.40 \mathrm{mmol}), 2 \mathrm{a}(0.60 \mathrm{mmol})$, catalyst III $(0.040 \mathrm{mmol})$ and $\mathrm{CH}_{2} \mathrm{Cl}_{2}(2.0 \mathrm{~mL})$ at $10{ }^{\circ} \mathrm{C}$ for 7 days to provide the title compound as a white solid (73\% yield, 90\% ee, 85:15 dr). ${ }^{1} \mathbf{H}$ NMR $\left(600 \mathrm{MHz}, \mathrm{CDCl}_{3}\right) \delta$ (ppm) $8.13(\mathrm{~d}, J=8.3 \mathrm{~Hz}, 1 \mathrm{H}), 7.48-7.47(\mathrm{~m}, 1 \mathrm{H}), 7.44-7.41(\mathrm{~m}, 1 \mathrm{H}), 7.28-7.27(\mathrm{~m}, 1 \mathrm{H}), 7.06$ $-7.03(\mathrm{~m}, 2 \mathrm{H}), 6.24(\mathrm{~s}, 1 \mathrm{H}), 5.29(\mathrm{~d}, J=9.7 \mathrm{~Hz}, 1 \mathrm{H}), 4.27-4.23(\mathrm{~m}, 2 \mathrm{H}), 2.84(\mathrm{dd}, J=16.3,9.9$ $\mathrm{Hz}, 1 \mathrm{H}), 2.42(\mathrm{~d}, J=16.3 \mathrm{~Hz}, 1 \mathrm{H}), 1.55(\mathrm{~s}, 3 \mathrm{H}), 1.31(\mathrm{t}, J=7.1 \mathrm{~Hz}, 3 \mathrm{H}) ;{ }^{13} \mathrm{C}$ NMR $(150 \mathrm{MHz}$, $\left.\mathrm{CDCl}_{3}\right) \delta(\mathrm{ppm}) 168.62,158.61,157.00,149.08,145.60,128.51,124.65,120.56,118.90,117.87$, 117.71, 114.32, 114.15, 88.65, 76.73, 61.46, 34.63, 14.08, 12.39; HRMS (ESI): Calcd for $\mathrm{C}_{20} \mathrm{H}_{19} \mathrm{FN}_{4} \mathrm{O}_{5}[\mathrm{M}+\mathrm{Na}]:$ 437.1237. Found: 437.1231; $[\alpha]_{\mathrm{D}}{ }^{29}=26.66\left(\mathrm{c}=1.11, \mathrm{CHCl}_{3}\right)$; the enantiomeric excess was determined by chiral HPLC (Chiralpak AD column: hexane/2-propanol = $60 / 40,0.5 \mathrm{~mL} / \mathrm{min}, 254 \mathrm{~nm}$, $\left.\mathrm{t}_{\text {major }}=16.61 \mathrm{~min}, \mathrm{t}_{\text {minor }}=14.70 \mathrm{~min}\right)$.

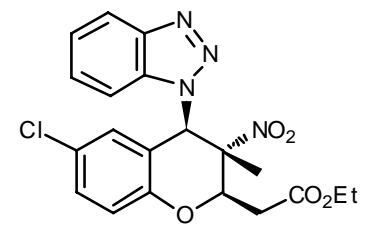

Ethyl 2-((2R,3S,4R)-4-(1H-benzo[d][1,2,3]triazol-1-yl)-6-chloro-3-methyl -3-nitrochroman-2-yl)acetate (3c). Prepared according to the general procedure from 1c $(0.40 \mathrm{mmol}), 2 \mathrm{a}(0.60 \mathrm{mmol})$, catalyst III $(0.040 \mathrm{mmol})$ and $\mathrm{CH}_{2} \mathrm{Cl}_{2}(2.0 \mathrm{~mL})$ at $10{ }^{\circ} \mathrm{C}$ for 5 days to provide the title compound as a white solid (77\% yield, 89\% ee, 90:10 dr). ${ }^{1} \mathbf{H}$ NMR $\left(600 \mathrm{MHz}, \mathrm{CDCl}_{3}\right) \delta$ (ppm) $8.14(\mathrm{~d}, J=8.3 \mathrm{~Hz}, 1 \mathrm{H}), 7.51-7.48(\mathrm{~m}, 1 \mathrm{H}), 7.45-7.42(\mathrm{~m}, 1 \mathrm{H}), 7.29-7.25(\mathrm{~m}, 3 \mathrm{H}), 7.01$ $(\mathrm{d}, J=8.8 \mathrm{~Hz}, 1 \mathrm{H}), 6.51(\mathrm{~s}, 1 \mathrm{H}), 5.30(\mathrm{~d}, J=9.7 \mathrm{~Hz}, 1 \mathrm{H}), 4.27-4.23(\mathrm{~m}, 2 \mathrm{H}), 2.85(\mathrm{dd}, J=16.3$, $9.9 \mathrm{~Hz}, 1 \mathrm{H}), 2.42(\mathrm{~d}, J=16.3 \mathrm{~Hz}, 1 \mathrm{H}), 1.56(\mathrm{~s}, 3 \mathrm{H}), 1.31(\mathrm{t}, J=7.1 \mathrm{~Hz}, 3 \mathrm{H}) ;{ }^{13} \mathrm{C}$ NMR $(150 \mathrm{MHz}$, $\left.\mathrm{CDCl}_{3}\right) \delta(\mathrm{ppm}) 168.51,151.47,145.49,130.56,128.50,127.81,127.56,124.65,120.49,118.85$, 88.46, 76.64, 61.43, 34.54, 14.06, 12.33; HRMS (ESI): Calcd for $\mathrm{C}_{20} \mathrm{H}_{19} \mathrm{ClN}_{4} \mathrm{O}_{5}$ [M+Na]: 453.0942 . Found: $453.0933 ;[\alpha]_{\mathrm{D}}{ }^{28}=52.25\left(\mathrm{c}=1.04, \mathrm{CHCl}_{3}\right)$; the enantiomeric excess was determined by chiral HPLC (Chiralpak AD column: hexane/2-propanol $=60 / 40,0.5 \mathrm{~mL} / \mathrm{min}, 254 \mathrm{~nm}, \mathrm{t}_{\text {major }}=$ $\left.21.81 \mathrm{~min}, \mathrm{t}_{\text {minor }}=16.32 \mathrm{~min}\right)$.

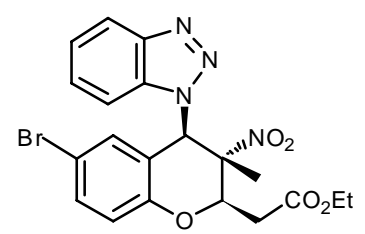

Ethyl 2-((2R,3S,4R)-4-(1H-benzo[d][1,2,3]triazol-1-yl)-6-bromo-3-methyl -3-nitrochroman-2-yl)acetate (3d). Prepared according to the general procedure from 1d $(0.40 \mathrm{mmol}), 2 \mathrm{a}(0.60 \mathrm{mmol})$, catalyst III $(0.040 \mathrm{mmol})$ and $\mathrm{CH}_{2} \mathrm{Cl}_{2}(2.0 \mathrm{~mL})$ at $10{ }^{\circ} \mathrm{C}$ for 5 days to provide the title compound as a white solid (76\% yield, 89\% ee, 89:11 dr). ${ }^{1} \mathbf{H}$ NMR $\left(600 \mathrm{MHz}, \mathrm{CDCl}_{3}\right) \delta$ (ppm) $8.13(\mathrm{~d}, J=8.3 \mathrm{~Hz}, 1 \mathrm{H}), 7.49-7.48(\mathrm{~m}, 1 \mathrm{H}), 7.44-7.41(\mathrm{~m}, 2 \mathrm{H}), 7.28-7.27(\mathrm{~m}, 1 \mathrm{H}), 6.95$ $(\mathrm{d}, J=8.8 \mathrm{~Hz}, 1 \mathrm{H}), 6.65(\mathrm{~s}, 1 \mathrm{H}), 5.30(\mathrm{~d}, J=9.8 \mathrm{~Hz}, 1 \mathrm{H}), 4.26-4.23(\mathrm{~m}, 2 \mathrm{H}), 2.85(\mathrm{dd}, J=16.3$, $9.9 \mathrm{~Hz}, 1 \mathrm{H}), 2.42(\mathrm{~d}, J=16.4,1 \mathrm{H}), 1.55(\mathrm{~s}, 3 \mathrm{H}), 1.30(\mathrm{t}, J=7.1 \mathrm{~Hz}, 3 \mathrm{H}) ;{ }^{13} \mathrm{C}$ NMR $(100 \mathrm{MHz}$, 
$\left.\mathrm{CDCl}_{3}\right) \delta(\mathrm{ppm}) 168.50,151.98,145.48,133.44,130.46,128.51,124.66,120.49,119.20,114.97$, 109.63, 88.41, 76.59, 62.14, 61.44, 34.53, 14.03, 12.33; HRMS (ESI): Calcd for $\mathrm{C}_{20} \mathrm{H}_{19} \mathrm{BrN}_{4} \mathrm{O}_{5}$ $[\mathrm{M}+\mathrm{Na}]$ : 497.0437. Found: 497.0419; $[\alpha]_{\mathrm{D}}{ }^{29}=51.08\left(\mathrm{c}=1.06, \mathrm{CHCl}_{3}\right)$; the enantiomeric excess was determined by chiral HPLC (Chiralpak AD column: hexane $/ 2-$ propanol $=60 / 40,0.5 \mathrm{~mL} / \mathrm{min}$, $\left.254 \mathrm{~nm}, \mathrm{t}_{\text {major }}=25.66 \mathrm{~min}, \mathrm{t}_{\text {minor }}=17.64 \mathrm{~min}\right)$.

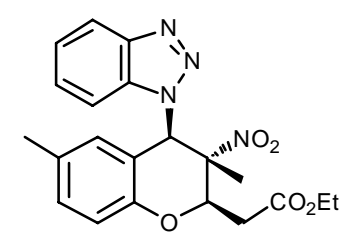

Ethyl 2-((2R,3S,4R)-4-(1H-benzo[d][1,2,3]triazol-1-yl)-3,6-dimethyl-3nitrochroman-2-yl)acetate (3e). Prepared according to the general procedure from 1e $(0.40 \mathrm{mmol})$, 2a $(0.60 \mathrm{mmol})$, catalyst $\mathbf{I I I}(0.040 \mathrm{mmol})$ and $\mathrm{CH}_{2} \mathrm{Cl}_{2}$ $(2.0 \mathrm{~mL})$ at $10{ }^{\circ} \mathrm{C}$ for 5 days to provide the title compound as a white solid (88\% yield, 90\% ee, 86:14 dr). ${ }^{1} \mathbf{H}$ NMR $\left(600 \mathrm{MHz}, \mathrm{CDCl}_{3}\right) \delta(\mathrm{ppm}) 8.12(\mathrm{~d}$, $J=8.1 \mathrm{~Hz}, 1 \mathrm{H}), 7.51-7.36(\mathrm{~m}, 2 \mathrm{H}), 7.29(\mathrm{~s}, 1 \mathrm{H}), 7.12(\mathrm{~d}, J=8.1 \mathrm{~Hz}, 1 \mathrm{H}), 6.95(\mathrm{~d}, J=8.3 \mathrm{~Hz}, 1 \mathrm{H})$, $6.34(\mathrm{~s}, 1 \mathrm{H}), 5.27(\mathrm{~d}, J=9.5 \mathrm{~Hz}, 1 \mathrm{H}), 4.26-4.23(\mathrm{~m}, 2 \mathrm{H}), 2.83(\mathrm{dd}, J=16.0,10.0 \mathrm{~Hz}, 1 \mathrm{H}), 2.41(\mathrm{~d}$, $J=16.1 \mathrm{~Hz}, 1 \mathrm{H}), 2.11(\mathrm{~s}, 3 \mathrm{H}), 1.52(\mathrm{~s}, 3 \mathrm{H}), 1.31(\mathrm{t}, J=7.0 \mathrm{~Hz}, 3 \mathrm{H}) ;{ }^{13} \mathbf{C} \mathbf{N M R}\left(100 \mathrm{MHz}, \mathrm{CDCl}_{3}\right)$ $\delta$ (ppm) 168.69, 150.82, 145.58, 133.61, 132.20, 131.20, 128.09, 127.90, 124.38, 120.34, 117.17, 116.72, 110.10, 89.01, 76.38, 63.04, 61.30, 34.67, 20.40, 14.03, 12.29; HRMS (ESI): Calcd for $\mathrm{C}_{21} \mathrm{H}_{22} \mathrm{~N}_{4} \mathrm{O}_{5}[\mathrm{M}+\mathrm{Na}]:$ 433.1488. Found: 433.1475; $[\alpha]_{\mathrm{D}}{ }^{29}=55.38\left(\mathrm{c}=1.22, \mathrm{CHCl}_{3}\right)$; the enantiomeric excess was determined by chiral HPLC (Chiralpak AD column: hexane/2-propanol = $60 / 40,0.5 \mathrm{~mL} / \mathrm{min}, 254 \mathrm{~nm}, \mathrm{t}_{\text {major }}=18.99 \mathrm{~min}, \mathrm{t}_{\text {minor }}=13.43 \mathrm{~min}$ ).

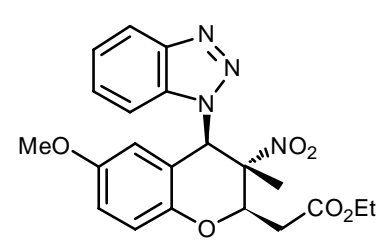

Ethyl 2-((2R,3S,4R)-4-(1H-benzo[d][1,2,3]triazol-1-yl)-6-methoxy-3methyl-3-nitrochroman-2-yl)acetate (3f). Prepared according to the general procedure from 1f $(0.40 \mathrm{mmol}), 2 \mathrm{a}(0.60 \mathrm{mmol})$, catalyst III $(0.040$ mmol) and $\mathrm{CH}_{2} \mathrm{Cl}_{2}(2.0 \mathrm{~mL})$ at $10{ }^{\circ} \mathrm{C}$ for 5 days to provide the title compound as a white solid (82\% yield, 90\% ee, 95:5 dr). ${ }^{1} \mathbf{H}$ NMR (600 $\left.\mathrm{MHz}, \mathrm{CDCl}_{3}\right) \delta(\mathrm{ppm}) 8.12(\mathrm{~d}, J=8.3 \mathrm{~Hz}, 1 \mathrm{H}), 7.43-7.39(\mathrm{~m}, 2 \mathrm{H}), 7.31-7.28(\mathrm{~m}, 1 \mathrm{H}), 7.00-$ $6.99(\mathrm{~m}, 1 \mathrm{H}), 6.90-6.89(\mathrm{~m}, 1 \mathrm{H}), 6.03(\mathrm{~s}, 1 \mathrm{H}), 5.25(\mathrm{~d}, J=9.7 \mathrm{~Hz}, 1 \mathrm{H}), 4.27-4.23(\mathrm{~m}, 2 \mathrm{H}), 3.51$ (s, 3H), $2.82(\mathrm{dd}, J=16.1,9.9 \mathrm{~Hz}, 1 \mathrm{H}), 2.39(\mathrm{~d}, J=16.1 \mathrm{~Hz}, 1 \mathrm{H}), 1.50(\mathrm{~s}, 3 \mathrm{H}), 1.31(\mathrm{t}, J=7.1 \mathrm{~Hz}$, $3 \mathrm{H}) ;{ }^{13} \mathrm{C}$ NMR $\left(100 \mathrm{MHz}, \mathrm{CDCl}_{3}\right) \delta$ (ppm) 13C NMR (101 MHz, cdcl3) $\delta 168.75,154.85,146.96$, $145.64,133.39,128.20,124.45,120.43,118.42,117.05,111.88,110.20,89.14,76.48,63.26,61.37$, 55.59, 34.66, 14.07, 12.34; HRMS (ESI): Calcd for $\mathrm{C}_{21} \mathrm{H}_{22} \mathrm{~N}_{4} \mathrm{O}_{6}[\mathrm{M}+\mathrm{Na}]$ : 449.1437. Found: $449.1425 ;[\alpha]_{\mathrm{D}}{ }^{28}=39.81\left(\mathrm{c}=1.01, \mathrm{CHCl}_{3}\right)$; the enantiomeric excess was determined by chiral HPLC (Chiralpak AD column: hexane/2-propanol $=60 / 40,0.5 \mathrm{~mL} / \mathrm{min}, 254 \mathrm{~nm}, \mathrm{t}_{\text {major }}=22.92 \mathrm{~min}$, $\left.\mathrm{t}_{\text {minor }}=19.28 \mathrm{~min}\right)$.

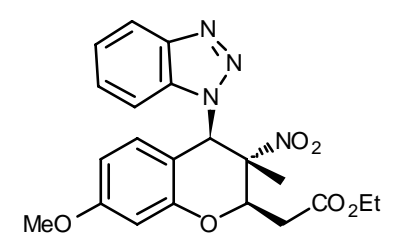

Ethyl 2-((2R,3S,4R)-4-(1H-benzo[d][1,2,3]triazol-1-yl)-7-methoxy-3methyl-3-nitrochroman-2-yl)acetate (3g). Prepared according to the general procedure from $1 \mathrm{~g}(0.40 \mathrm{mmol})$, 2a $(0.60 \mathrm{mmol})$, catalyst III $(0.040$ mmol) and $\mathrm{CH}_{2} \mathrm{Cl}_{2}(2.0 \mathrm{~mL})$ at $10{ }^{\circ} \mathrm{C}$ for 5 days to provide the title compound as a white solid (82\% yield, $91 \%$ ee, 90:10 dr). ${ }^{1} \mathbf{H}$ NMR (600 $\left.\mathrm{MHz}, \mathrm{CDCl}_{3}\right) \delta(\mathrm{ppm}) 8.10(\mathrm{~d}, J=8.1 \mathrm{~Hz}, 1 \mathrm{H}), 7.46-7.35(\mathrm{~m}, 2 \mathrm{H}), 7.28(\mathrm{~s}, 1 \mathrm{H}), 7.02(\mathrm{~s}, 1 \mathrm{H}), 6.58$ 
(s, 1H), $6.58(\mathrm{~s}, 1 \mathrm{H}), 6.52-6.48(\mathrm{~m}, 2 \mathrm{H}), 5.30(\mathrm{~d}, J=8.9 \mathrm{~Hz}, 1 \mathrm{H}), 4.27-4.24(\mathrm{~m}, 2 \mathrm{H}), 3.80(\mathrm{~s}$, $3 \mathrm{H}), 2.82(\mathrm{dd}, J=16.2,10.0 \mathrm{~Hz}, 1 \mathrm{H}), 2.41(\mathrm{~d}, J=15.2 \mathrm{~Hz}, 1 \mathrm{H}), 1.48(\mathrm{~s}, 3 \mathrm{H}), 1.32(\mathrm{t}, J=7.1 \mathrm{~Hz}$, $3 \mathrm{H}) ;{ }^{13} \mathrm{C}$ NMR $\left(150 \mathrm{MHz}, \mathrm{CDCl}_{3}\right) \delta$ (ppm) 168.72, 161.18, 154.06, 145.73, 133.28, 128.95, 128.04, $124.34,120.39,110.15,101.69,89.05,76.45,61.40,55.44,34.64,14.07,12.33,12.24$; HRMS (ESI): Calcd for $\mathrm{C}_{21} \mathrm{H}_{22} \mathrm{~N}_{4} \mathrm{O}_{6}[\mathrm{M}+\mathrm{Na}]$ : 449.1437. Found: 449.1417; $[\alpha]_{\mathrm{D}}{ }^{29}=44.07(\mathrm{c}=1.02$, $\mathrm{CHCl}_{3}$ ); the enantiomeric excess was determined by chiral HPLC (Chiralpak AD column: hexane/2-propanol $=60 / 40,0.5 \mathrm{~mL} / \mathrm{min}, 254 \mathrm{~nm}, \mathrm{t}_{\text {major }}=19.20 \mathrm{~min}, \mathrm{t}_{\text {minor }}=17.57 \mathrm{~min}$ ).

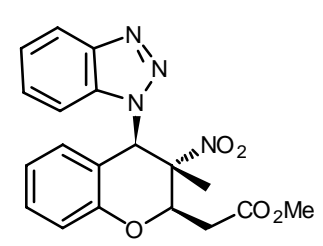

Methyl 2-((2R,3S,4R)-4-(1H-benzo[d][1,2,3]triazol-1-yl)-3-methyl-3-nitro chroman-2-yl)acetate (3h). Prepared according to the general procedure from 1b $(0.40 \mathrm{mmol}), \mathbf{2 h}(0.60 \mathrm{mmol})$, catalyst III $(0.040 \mathrm{mmol})$ and $\mathrm{CH}_{2} \mathrm{Cl}_{2}(2.0$ $\mathrm{mL})$ at $10{ }^{\circ} \mathrm{C}$ for 5 days to provide the title compound as a white solid $(84 \%$ yield, 92\% ee, 92:8 dr). ${ }^{1} \mathbf{H}$ NMR $\left(600 \mathrm{MHz}, \mathrm{CDCl}_{3}\right) \delta(\mathrm{ppm}) 8.12-8.10(\mathrm{~m}$, $1 \mathrm{H}), 7.44-7.36(\mathrm{~m}, 2 \mathrm{H}), 7.33-7.28(\mathrm{~m}, 2 \mathrm{H}), 7.07-7.06(\mathrm{~m}, 1 \mathrm{H}), 6.93-6.91(\mathrm{~m}, 1 \mathrm{H}), 6.55(\mathrm{~s}$, $1 \mathrm{H}), 5.32(\mathrm{~d}, J=9.8 \mathrm{~Hz}, 1 \mathrm{H}), 3.79(\mathrm{~s}, 3 \mathrm{H}), 2.86(\mathrm{dd}, J=16.1,10.0 \mathrm{~Hz}, 1 \mathrm{H}), 2.43(\mathrm{~d}, J=16.3 \mathrm{~Hz}$, 1H), 1.53 (s, 3H); ${ }^{13} \mathrm{C}$ NMR (150 MHz, $\left.\mathrm{CDCl}_{3}\right) \delta$ (ppm) 169.06, 152.78, 145.55, 130.33, 127.97, $124.29,122.53,120.25,117.33,110.13,88.73,76.20,63.09,52.23,34.31,12.24$; HRMS (ESI): Calcd for $\mathrm{C}_{19} \mathrm{H}_{18} \mathrm{~N}_{4} \mathrm{O}_{5}[\mathrm{M}+\mathrm{Na}]: 405.1175$. Found: 405.1164. $[\alpha]_{\mathrm{D}}{ }^{29}=41.53\left(\mathrm{c}=1.07 \mathrm{CHCl}_{3}\right)$; the enantiomeric excess was determined by chiral HPLC (Chiralpak AS column: hexane/2-propanol = $60 / 40,0.5 \mathrm{~mL} / \mathrm{min}, 254 \mathrm{~nm}, \mathrm{t}_{\text {major }}=39.99 \mathrm{~min}, \mathrm{t}_{\text {minor }}=28.73 \mathrm{~min}$ ).

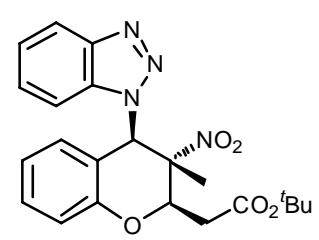

Tert-butyl 2-((2R,3S,4R)-4-(1H-benzo[d][1,2,3]triazol-1-yl)-3-methyl-3nitrochroman-2-yl)acetate (3i). Prepared according to the general procedure from $1 \mathbf{i}(0.40 \mathrm{mmol})$, 2a $(0.60 \mathrm{mmol})$, catalyst $\mathbf{I I I}(0.040 \mathrm{mmol})$ and $\mathrm{CH}_{2} \mathrm{Cl}_{2}$ $(2.0 \mathrm{~mL})$ at $10{ }^{\circ} \mathrm{C}$ for 7 days to provide the title compound as a white solid (91\% yield, 90\% ee, 86:14 dr). ${ }^{\mathbf{1}} \mathbf{H}$ NMR $\left(600 \mathrm{MHz}, \mathrm{CDCl}_{3}\right) \delta(\mathrm{ppm}) 8.11(\mathrm{~d}, J$ $=8.2 \mathrm{~Hz}, 1 \mathrm{H}), 7.42-7.38(\mathrm{~m}, 2 \mathrm{H}), 7.33-7.32(\mathrm{~m}, 2 \mathrm{H}), 7.05(\mathrm{~d}, J=8.2 \mathrm{~Hz}, 1 \mathrm{H}), 6.92(\mathrm{t}, J=7.6$ $\mathrm{Hz}, 1 \mathrm{H}), 6.55(\mathrm{~s}, 1 \mathrm{H}), 5.23(\mathrm{~d}, J=8.9 \mathrm{~Hz}, 1 \mathrm{H}), 2.74(\mathrm{dd}, J=15.9,9.9 \mathrm{~Hz}, 1 \mathrm{H}), 2.37(\mathrm{~d}, J=15.2 \mathrm{~Hz}$, 1H), $1.51(\mathrm{~s}, 12 \mathrm{H}) ;{ }^{13} \mathrm{C}$ NMR $\left(150 \mathrm{MHz}, \mathrm{CDCl}_{3}\right) \delta(\mathrm{ppm}) 167.73,153.01,145.63,130.39,128.07$, $124.35,122.57,120.35,117.35,88.84,81.93,76.72,63.06,35.75,27.94,12.34$; HRMS (ESI): Calcd for $\mathrm{C}_{22} \mathrm{H}_{24} \mathrm{~N}_{4} \mathrm{O}_{5}[\mathrm{M}+\mathrm{Na}]$ : 447.1644. Found: 447.1639; $[\alpha]_{\mathrm{D}}{ }^{29}=35.68\left(\mathrm{c}=1.00, \mathrm{CHCl}_{3}\right)$; the enantiomeric excess was determined by chiral HPLC (Chiralpak AD column: hexane/2-propanol = $\left.60 / 40,0.5 \mathrm{~mL} / \mathrm{min}, 254 \mathrm{~nm}, \mathrm{t}_{\text {major }}=11.09 \mathrm{~min}, \mathrm{t}_{\text {minor }}=12.10 \mathrm{~min}\right)$.

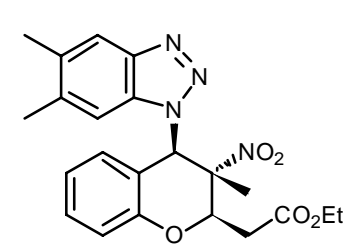

Ethyl 2-((2R,3S,4R)-4-(5,6-dimethyl-1H-benzo[d][1,2,3]triazol-1-yl)-3methyl-3-nitrochroman-2-yl)acetate $(3 \mathbf{j})$. Prepared according to the general procedure from 1a $(0.40 \mathrm{mmol}), 2 \mathbf{b}(0.60 \mathrm{mmol})$, catalyst III $(0.040 \mathrm{mmol})$ and $\mathrm{CH}_{2} \mathrm{Cl}_{2}(2.0 \mathrm{~mL})$ at $10{ }^{\circ} \mathrm{C}$ for 5 days to provide the title compound as a white solid (90\% yield, 96\% ee, 96:4 dr) ${ }^{\mathbf{1}} \mathbf{H}$ NMR (600 MHz, $\left.\mathrm{CDCl}_{3}\right) \delta(\mathrm{ppm})$ $7.81(\mathrm{~s}, 1 \mathrm{H}), 7.32-7.25(\mathrm{~m}, 2 \mathrm{H}), 7.04(\mathrm{~d}, J=8.1 \mathrm{~Hz}, 1 \mathrm{H}), 6.90(\mathrm{t}, J=7.5 \mathrm{~Hz}, 1 \mathrm{H}), 6.52(\mathrm{~s}, 1 \mathrm{H})$, $5.30(\mathrm{~d}, J=9.8 \mathrm{~Hz}, 1 \mathrm{H}), 4.26-4.23(\mathrm{~m}, 2 \mathrm{H}), 2.84(\mathrm{dd}, J=16.1,9.9 \mathrm{~Hz}, 1 \mathrm{H}), 2.42(\mathrm{~d}, J=16.1 \mathrm{~Hz}$, 
1H), $2.38(\mathrm{~s}, 3 \mathrm{H}), 2.32(\mathrm{~s}, 3 \mathrm{H}), 1.55(\mathrm{~s}, 3 \mathrm{H}), 1.31(\mathrm{t}, J=7.1 \mathrm{~Hz}, 3 \mathrm{H}) ;{ }^{13} \mathbf{C} \mathbf{N M R}\left(150 \mathrm{MHz}, \mathrm{CDCl}_{3}\right)$ $\delta(\mathrm{ppm}) 168.67,152.80,144.83,138.45,134.23,132.76,130.24,128.03,122.51,119.28,117.26$, 109.48, 88.80, 76.39, 62.92, 61.29, 34.66, 20.92, 20.17, 14.01, 12.29; HRMS (MALDI); Calcd for. $\mathrm{C}_{22} \mathrm{H}_{24} \mathrm{~N}_{4} \mathrm{O}_{5}[\mathrm{M}+\mathrm{H}]: 425.1825$; Found: 425.1833; $[\alpha]_{\mathrm{D}}{ }^{27}=24.95\left(\mathrm{c}=1.03, \mathrm{CHCl}_{3}\right)$; the enantiomeric excess was determined by chiral HPLC (Chiralpak AS column: hexane/2-propanol = $\left.60 / 40,0.5 \mathrm{~mL} / \mathrm{min}, 254 \mathrm{~nm}, \mathrm{t}_{\text {major }}=38.47 \mathrm{~min}, \mathrm{t}_{\text {minor }}=21.99 \mathrm{~min}\right)$.

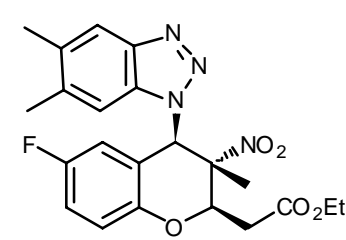

Ethyl 2-((2R,3S,4R)-4-(5,6-dimethyl-1H-benzo[d][1,2,3]triazol-1-yl)-6fluoro-methyl-3-nitrochroman-2-yl)acetate (3k). Prepared according to the general procedure from $\mathbf{1 b}(0.40 \mathrm{mmol}), \mathbf{2 b}(0.60 \mathrm{mmol})$, catalyst III $(0.040$ $\mathrm{mmol})$ and $\mathrm{CH}_{2} \mathrm{Cl}_{2}(2.0 \mathrm{~mL})$ at $10{ }^{\circ} \mathrm{C}$ for 7 days to provide the title compound as a white solid (82\% yield, 91\% ee, 90:10 dr). ${ }^{1} \mathbf{H}$ NMR (600 MHz, $\left.\mathrm{CDCl}_{3}\right)$ $\delta(\mathrm{ppm}) 7.85(\mathrm{~s}, 1 \mathrm{H}), 7.18(\mathrm{~s}, 1 \mathrm{H}), 7.02(\mathrm{~s}, 2 \mathrm{H}), 6.20(\mathrm{~s}, 1 \mathrm{H}), 5.27(\mathrm{~d}, J=9.6 \mathrm{~Hz}, 1 \mathrm{H}), 4.27-4.24$ (m, 2H), $2.84(\mathrm{dd}, J=15.9,10.0 \mathrm{~Hz}, 1 \mathrm{H}), 2.44(\mathrm{~d}, J=14.1 \mathrm{~Hz}, 1 \mathrm{H}), 2.40$ (s, 3H), 2.37 (s, 3H), 1.57 $(\mathrm{s}, 3 \mathrm{H}), 1.32(\mathrm{t}, J=7.0 \mathrm{~Hz}, 3 \mathrm{H}) ;{ }^{13} \mathrm{C}$ NMR $\left(150 \mathrm{MHz}, \mathrm{CDCl}_{3}\right) \delta(\mathrm{ppm}) 168.68,158.54,156.93$, 148.93, 144.66, 139.05, 134.58, 119.41, 118.71, 117.67, 117.51, 114.26, 114.09, 88.59, 76.70, 61.42, 34.60, 21.02, 20.27, 14.04, 12.38; HRMS (MALDI); Calcd for $\mathrm{C}_{22} \mathrm{H}_{23} \mathrm{FN}_{4} \mathrm{O}_{5}[\mathrm{M}+\mathrm{H}]$ : 443.1731; Found: $443.1743 ;[\alpha]_{\mathrm{D}}{ }^{28}=14.27\left(\mathrm{c}=1.03, \mathrm{CHCl}_{3}\right)$; the enantiomeric excess was determined by chiral HPLC (Chiralpak AS column: hexane/2-propanol $=60 / 40,0.5 \mathrm{~mL} / \mathrm{min}, 254 \mathrm{~nm}, \mathrm{t}_{\text {major }}=35.55$ $\left.\min , \mathrm{t}_{\text {minor }}=16.49 \mathrm{~min}\right)$.

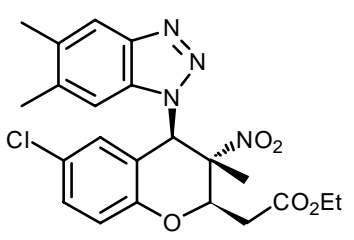

Ethyl 2-((2R,3S,4R)-6-chloro-4-(5,6-dimethyl-1H-benzo[d][1,2,3]triazol1-yl)-3-methyl-3-nitrochroman-2-yl)acetate (3l). Prepared according to the general procedure from 1c $(0.40 \mathrm{mmol}), 2 \mathbf{b}(0.60 \mathrm{mmol})$, catalyst III $(0.040$ $\mathrm{mmol})$ and $\mathrm{CH}_{2} \mathrm{Cl}_{2}(2.0 \mathrm{~mL})$ at $10{ }^{\circ} \mathrm{C}$ for 5 days to provide the title compound as a white solid (80\% yield, 90\% ee, 90:10 dr). ${ }^{1} \mathbf{H}$ NMR (600 $\left.\mathrm{MHz}, \mathrm{CDCl}_{3}\right) \delta(\mathrm{ppm}) 7.85(\mathrm{~s}, 1 \mathrm{H}), 7.27(\mathrm{~s}, 2 \mathrm{H}), 7.15(\mathrm{~s}, 1 \mathrm{H}), 6.99(\mathrm{~d}, J=8.6 \mathrm{~Hz}, 1 \mathrm{H}), 6.45(\mathrm{~s}, 1 \mathrm{H})$, $5.28(\mathrm{~d}, J=9.7 \mathrm{~Hz}, 1 \mathrm{H}), 4.27-4.24(\mathrm{~m}, 2 \mathrm{H}), 2.84(\mathrm{dd}, J=16.1,9.9 \mathrm{~Hz}, 1 \mathrm{H}), 2.44(\mathrm{~d}, J=13.8 \mathrm{~Hz}$, 1H), $2.41(\mathrm{~s}, 3 \mathrm{H}), 2.39$ (s, 3H), 1.59 (s, 3H), $1.31(\mathrm{t}, J=7.0 \mathrm{~Hz}, 3 \mathrm{H}) ;{ }^{13} \mathbf{C} \mathbf{N M R}\left(150 \mathrm{MHz}, \mathrm{CDCl}_{3}\right)$ $\delta(\mathrm{ppm}) 168.61,151.35,144.66,139.13,134.63,130.43,127.77,127.53,119.42,118.76,88.44$, 76.68, 61.44, 34.58, 21.05, 20.31, 14.05, 12.39; HRMS (MALDI); Calcd for $\mathrm{C}_{22} \mathrm{H}_{23} \mathrm{ClN}_{4} \mathrm{O}_{5}[\mathrm{M}+\mathrm{H}]$ : 459.1435 Found: 459.1445; $[\alpha]_{\mathrm{D}}{ }^{29}=39.77\left(\mathrm{c}=1.24, \mathrm{CHCl}_{3}\right)$; the enantiomeric excess was determined by chiral HPLC (Chiralpak AS column: hexane/2-propanol $=60 / 40,0.5 \mathrm{~mL} / \mathrm{min}, 254$ $\left.\mathrm{nm}, \mathrm{t}_{\text {major }}=30.56 \mathrm{~min}, \mathrm{t}_{\text {minor }}=16.44 \mathrm{~min}\right)$.

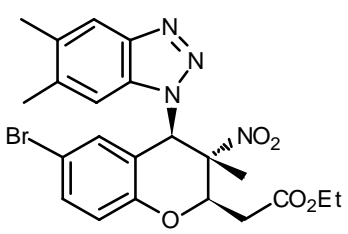

Ethyl 2-((2R,3S,4R)-6-bromo-4-(5,6-dimethyl-1H-benzo[d][1,2,3]triazol1-yl)-3-methyl-3-nitrochroman-2-yl)acetate $\mathbf{( 3 m )}$. Prepared according to the general procedure from $\mathbf{1 d}(0.40 \mathrm{mmol}), \mathbf{2 b}(0.60 \mathrm{mmol})$, catalyst III $(0.040 \mathrm{mmol})$ and $\mathrm{CH}_{2} \mathrm{Cl}_{2}(2.0 \mathrm{~mL})$ at $10{ }^{\circ} \mathrm{C}$ for 5 days to provide the title compound as a white solid (70\% yield, 91\% ee, 90:10 dr). ${ }^{\mathbf{1}} \mathbf{H}$ NMR (600 $\left.\mathrm{MHz}, \mathrm{CDCl}_{3}\right) \delta(\mathrm{ppm}) 7.85(\mathrm{~s}, 1 \mathrm{H}), 7.41(\mathrm{~d}, J=8.5 \mathrm{~Hz}, 1 \mathrm{H}), 7.15(\mathrm{~s}, 1 \mathrm{H}), 6.93(\mathrm{~d}, J=8.6 \mathrm{~Hz}, 1 \mathrm{H})$, 
$6.60(\mathrm{~s}, 1 \mathrm{H}), 5.28(\mathrm{~d}, J=9.7 \mathrm{~Hz}, 1 \mathrm{H}), 4.27-4.23(\mathrm{~m}, 2 \mathrm{H}), 2.84(\mathrm{dd}, J=16.1,9.9 \mathrm{~Hz}, 1 \mathrm{H}), 2.43(\mathrm{~s}$, 1H), $2.41(\mathrm{~s}, 3 \mathrm{H}), 2.39$ (s, 3H), 1.59 (s, 3H), 1.31 (t, $J=7.0 \mathrm{~Hz}, 3 \mathrm{H}) ;{ }^{13} \mathbf{C}$ NMR $\left(150 \mathrm{MHz}, \mathrm{CDCl}_{3}\right)$ $\delta$ (ppm) 168.56, 151.83, 144.57, 139.08, 137.55, 134.59, 133.26, 132.90, 130.40, 125.18, 119.38, $119.08,114.90,88.36,76.61,61.41,34.54,21.03,20.28,20.22,14.04,12.35$; HRMS (MALDI); Calcd for $\mathrm{C}_{22} \mathrm{H}_{23} \mathrm{BrN}_{4} \mathrm{O}_{5}[\mathrm{M}+\mathrm{H}]$ : 503.0930; Found: 503.0945; $[\alpha]_{\mathrm{D}}{ }^{29}=38.58\left(\mathrm{c}=1.39, \mathrm{CHCl}_{3}\right)$; the enantiomeric excess was determined by chiral HPLC (Chiralpak AS column: hexane/2-propanol = $60 / 40,0.5 \mathrm{~mL} / \mathrm{min}, 254 \mathrm{~nm}, \mathrm{t}_{\text {major }}=32.29 \mathrm{~min}, \mathrm{t}_{\text {minor }}=17.94 \mathrm{~min}$ ).

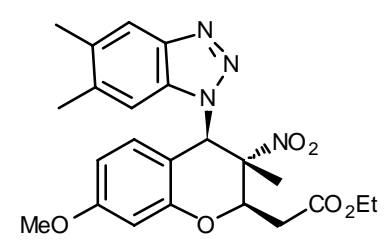

Ethyl 2-((2R,3S,4R)-4-(5,6-dimethyl-1H-benzo[d][1,2,3]triazol-1-yl)-7 -methoxy-3-methyl-3-nitrochroman-2-yl)acetate (3n). Prepared according to the general procedure from $1 \mathbf{g}(0.40 \mathrm{mmol}), \mathbf{2 b}(0.60 \mathrm{mmol})$, catalyst III $(0.040 \mathrm{mmol})$ and $\mathrm{CH}_{2} \mathrm{Cl}_{2}(2.0 \mathrm{~mL})$ at $10{ }^{\circ} \mathrm{C}$ for 5 days to provide the title compound as a white solid ( $85 \%$ yield, 93\% ee, $91: 9 \mathrm{dr})$. ${ }^{1}$ H NMR $\left(600 \mathrm{MHz}, \mathrm{CDCl}_{3}\right) \delta(\mathrm{ppm}) 7.82(\mathrm{~s}, 1 \mathrm{H}), 7.27-7.18(\mathrm{~m}, 2 \mathrm{H}), 6.56(\mathrm{~s}, 1 \mathrm{H}), 6.51-6.50(\mathrm{~m}$, 1H), $6.44(\mathrm{~s}, 1 \mathrm{H}), 5.29(\mathrm{~d}, J=9.8 \mathrm{~Hz}, 1 \mathrm{H}), 4.26-4.25(\mathrm{~m}, 2 \mathrm{H}), 3.80(\mathrm{~s}, 3 \mathrm{H}), 2.84-2.80(\mathrm{~m}, 1 \mathrm{H})$, $2.42(\mathrm{~s}, 1 \mathrm{H}), 2.39(\mathrm{~s}, 3 \mathrm{H}), 2.33(\mathrm{~s}, 3 \mathrm{H}), 1.51(\mathrm{~s}, 3 \mathrm{H}), 1.32(\mathrm{t}, J=5.4 \mathrm{~Hz}, 3 \mathrm{H}) ;{ }^{13} \mathrm{C}$ NMR $(150 \mathrm{MHz}$, $\left.\mathrm{CDCl}_{3}\right) \delta(\mathrm{ppm}) 168.70,160.99,153.84,144.82,138.45,134.17,128.82,119.22,109.95,101.52$, 88.95, 76.40, 61.29, 55.34, 34.58, 20.88, 20.21, 20.14, 14.03, 12.21; HRMS (MALDI); Calcd for $\mathrm{C}_{23} \mathrm{H}_{26} \mathrm{~N}_{4} \mathrm{O}_{6}[\mathrm{M}+\mathrm{Na}]: 477.1750$; Found: 477.1766; $[\alpha]_{\mathrm{D}}{ }^{28}=34.65$ (c $=1.09, \mathrm{CHCl}_{3}$; the enantiomeric excess was determined by chiral HPLC (Chiralpak AS column: hexane/2-propanol = $60 / 40,0.5 \mathrm{~mL} / \mathrm{min}, 254 \mathrm{~nm}$, $\mathrm{t}_{\text {major }}=53.93 \mathrm{~min}$, $\mathrm{t}_{\text {minor }}=26.65 \mathrm{~min}$ ).

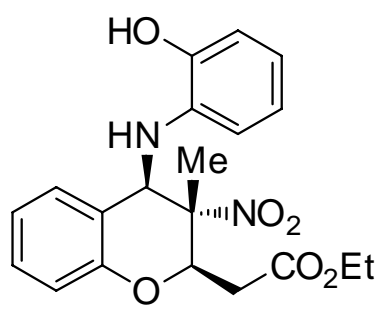

2-((2R,3S,4R)-4-((2-hydroxyphenyl)amino)-3-methyl-3-nitro-1,2,3,4-tetra hydronaphthalen-2-yl)acetate (3o)

Prepared according to the general procedure from 1a $(0.40 \mathrm{mmol})$, 2-aminophenol $(0.60 \mathrm{mmol}), \mathrm{CH}_{2} \mathrm{Cl}_{2}(2.0 \mathrm{~mL})$ at $\mathrm{rt}$ for $48 \mathrm{~h}$ to provide the title compound as a pale yellow oil (67\% yield, $86 \%$ ee, $97: 3$ d.r.). ${ }^{1} \mathbf{H}$ NMR $\left(400 \mathrm{MHz}, \mathrm{CDCl}_{3}\right) \delta(\mathrm{ppm}) 7.17-7.25(\mathrm{~m}, 1 \mathrm{H}), 6.93(\mathrm{t}, J=7.2 \mathrm{~Hz}, 1 \mathrm{H})$, 6.75-6.84 (m, 3H), 6.61-6.73 (m, 2H), 5.76-5.78 (m, 2H), $5.17(\mathrm{dd}, J=9.6,2.4 \mathrm{~Hz}, 1 \mathrm{H}), 4.21-4.26$ (m, 2H), $2.75(\mathrm{dd}, J=16.0,10.0 \mathrm{~Hz}, 2 \mathrm{H}), 2.39$ (d, $J=16.0 \mathrm{~Hz}, 1 \mathrm{H}), 1.57(\mathrm{~s}, 3 \mathrm{H}), 1.30(\mathrm{t}, J=7.2$ $\mathrm{Hz}, 3 \mathrm{H}) .{ }^{13} \mathrm{C}$ NMR $\left(100 \mathrm{MHz}, \mathrm{CDCl}_{3}\right) \delta(\mathrm{ppm}) 169.53,152.30,142.81,135.74,129.42,127.39$, $123.57,122.35,121,87,118.70,116.48,114.74,112.02,88.57,76.43,61.44,59.43,35.10,14.10$, 10.49. HRMS (ESI): Calcd for $\mathrm{C}_{20} \mathrm{H}_{23} \mathrm{NO}_{5} \mathrm{~S}[\mathrm{M}+\mathrm{Na}]$ : 409.1376. Found: 409.1352. $[\alpha]_{\mathrm{D}}^{29}=-8.28(C$ $\left.=1.00, \mathrm{CHCl}_{3}\right)$. HPLC (Chiralpak AD-H column, hexane $/ 2$-propanol $=85: 15,1.0 \mathrm{~mL} / \mathrm{min} ; 254 \mathrm{~nm}$, $\left.25{ }^{\circ} \mathrm{C}, \mathrm{t}_{1}=8.06 \mathrm{~min}, \mathrm{t}_{2}=9.69 \mathrm{~min}, \mathrm{t}_{3}=11.71 \mathrm{~min}, \mathrm{t}_{2}=15.56 \mathrm{~min}\right)$.

\section{Synthesis transformation of the cascade reaction adduct 3a}




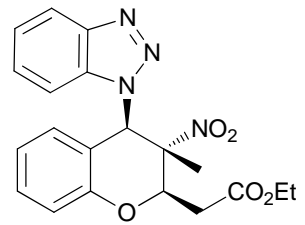

3a

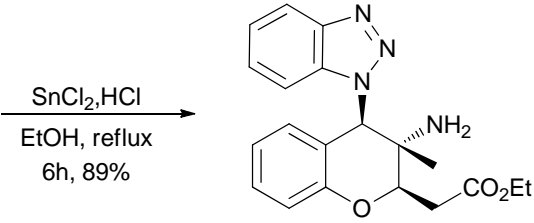

$4 \mathbf{a}$

Ethyl 2-((2R,3S,4R)-3-amino-4-(1H-benzo[d][1,2,3]triazol-1-yl)-3-methylchroman-2-yl)acetate (4a). To a two-necked 10-mL flask were sequentially added (1.89 g, $10 \mathrm{mmol})$ of anhydrous tin(II) chloride in Glove Box and a solution of 3a $(0.40 \mathrm{~g}, 1.0 \mathrm{mmol})$ in $12.5 \mathrm{~mL}$ of EtOH containing (10 mmol $\mathrm{HCl}$, prepared from $10 \mathrm{mmol}$ of acetic chloride at $0{ }^{\circ} \mathrm{C}$ ) under nitrogen atmosphere. The reaction mixture was heated to reflux for $4 \mathrm{~h}$. It was then cooled to $0{ }^{\circ} \mathrm{C}$, quenched with sat. $\mathrm{NH}_{4} \mathrm{Cl}$ and basified with $\mathrm{NH}_{3} \cdot \mathrm{H}_{2} \mathrm{O}$. Dichloromethane was added and vigorously stirred for $10 \mathrm{~min}$. The white suspension was suction filtrated over Celit. The aqueous phase was extracted with $\mathrm{CH}_{2} \mathrm{Cl}_{2}$ and the combined organic phase was dried over $\mathrm{Na}_{2} \mathrm{SO}_{4}$. Then the crude product was purified by flash silica gel chromatography to afford the product $\mathbf{4 a}$ as white solid in $89 \%$ yield. ${ }^{\mathbf{1}} \mathbf{H} \mathbf{~ N M R}(600$ $\left.\mathrm{MHz}, \mathrm{CDCl}_{3}\right) \delta(\mathrm{ppm}) 8.09(\mathrm{~d}, J=8.3 \mathrm{~Hz}, 1 \mathrm{H}), 7.34-7.30(\mathrm{~m}, 1 \mathrm{H}), 7.27-7.24(\mathrm{~m}, 2 \mathrm{H}), 7.00(\mathrm{~d}, J$ $=8.1 \mathrm{~Hz}, 1 \mathrm{H}), 6.81(\mathrm{t}, J=7.4 \mathrm{~Hz}, 1 \mathrm{H}), 6.56(\mathrm{~s}, 1 \mathrm{H}), 6.31(\mathrm{~s}, 1 \mathrm{H}), 4.73(\mathrm{~d}, J=6.3 \mathrm{~Hz}, 1 \mathrm{H}), 4.26-$ $4.24(\mathrm{~m}, 2 \mathrm{H}), 3.14(\mathrm{~d}, J=15.9 \mathrm{~Hz}, 1 \mathrm{H}), 2.73(\mathrm{dd}, J=15.9,9.0 \mathrm{~Hz}, 1 \mathrm{H}), 1.57(\mathrm{~s}, 2 \mathrm{H}), 1.31(\mathrm{t}, J=$ $7.1 \mathrm{~Hz}, 3 \mathrm{H}), 0.89$ (s, 3H); ${ }^{13} \mathbf{C}$ NMR (150 MHz, $\left.\mathrm{CDCl}_{3}\right) \delta(\mathrm{ppm}) 171.21,154.35,146.15,129.72$, 128.23, 126.93, 123.72, 121.32, 120.01, 117.22, 79.62, 60.77, 60.22, 53.09, 34.70, 16.94, 14.14; HRMS (MALDI); Calcd for $\mathrm{C}_{20} \mathrm{H}_{22} \mathrm{~N}_{4} \mathrm{O}_{3}[\mathrm{M}+\mathrm{Na}]$ : 389.1590; Found: 389.1591 .

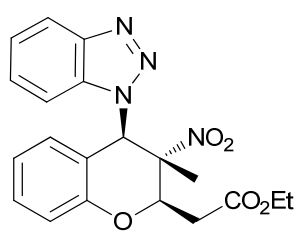

$3 \mathbf{a}$

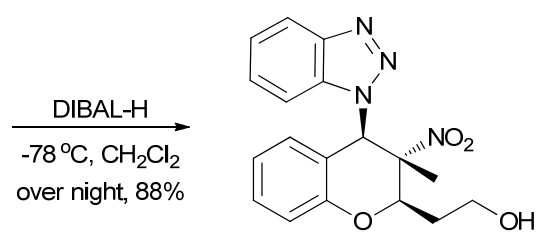

$5 \mathbf{a}$

2-((2R,3S,4R)-4-(1H-benzo[d][1,2,3]triazol-1-yl)-3-methyl-3-nitrochroman-2-yl)ethanol (5a).

To a solution of the nitro $3 \mathbf{a}(0.40 \mathrm{~g}, 1.0 \mathrm{mmol})$ in dichloromethane $(0.06 \mathrm{M})$ at $-78^{\circ} \mathrm{C}$, was added DIBAL-H (4.0 eq, 1M solution in hexane), the reaction was left to warm to room temperature overnight. The reaction was quenched with aqueous hydrochloric acid $(1.0 \mathrm{M})$ and water, the aqueous layer was extracted with dichloromethane. The combined organic layers were washed with water, dried over $\mathrm{MgSO}_{4}$, filtered and concentrated under reduced pressure to afford the alcohol 5a, which was purified by column chromatograph (petroleum ether / ethyl acetate $=5 / 1$ ) as a white solid in $88 \%$ yield. ${ }^{1} \mathbf{H}$ NMR $\left(600 \mathrm{MHz}, \mathrm{CDCl}_{3}\right) \delta(\mathrm{ppm}) 8.10(\mathrm{~d}, J=7.1 \mathrm{~Hz}, 1 \mathrm{H}), 7.38(\mathrm{~s}, 2 \mathrm{H})$, $7.35-7.26(\mathrm{~m}, 2 \mathrm{H}), 7.07-7.06(\mathrm{~m}, 1 \mathrm{H}), 6.91(\mathrm{t}, J=7.1 \mathrm{~Hz}, 1 \mathrm{H}), 6.56(\mathrm{~s}, 1 \mathrm{H}), 4.99(\mathrm{~d}, J=9.5 \mathrm{~Hz}$, $1 \mathrm{H}), 3.96(\mathrm{~s}, 2 \mathrm{H}), 2.56(\mathrm{~s}, 1 \mathrm{H}), 2.06-2.02(\mathrm{~m}, 1 \mathrm{H}), 1.65-1.61(\mathrm{~m}, 1 \mathrm{H}), 1.50(\mathrm{~s}, 3 \mathrm{H}) ;{ }^{13} \mathbf{C} \mathbf{N M R}$ $\left(150 \mathrm{MHz}, \mathrm{CDCl}_{3}\right) \delta(\mathrm{ppm}) 153.17,145.62,130.38,128.18,128.09,124.44,122.45,120.30,117.38$, 89.53, 77.03, 58.26, 31.61, 12.05; HRMS (MALDI); Calcd for $\mathrm{C}_{18} \mathrm{H}_{18} \mathrm{~N}_{4} \mathrm{O}_{4}$ [M+Na]: 377.1226; Found: 377.1226. 


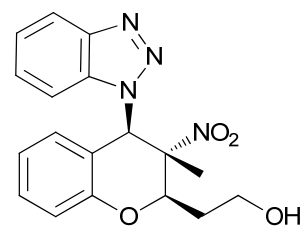

$5 a$

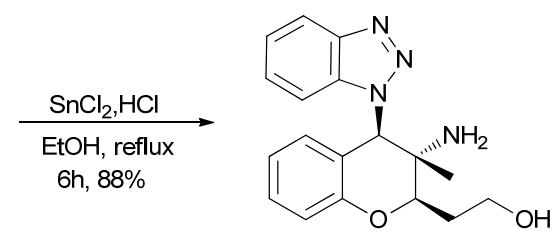

$6 \mathbf{a}$

2-((2R,3S,4R)-3-amino-4-(1H-benzo[d][1,2,3]triazol-1-yl)-3-methylchroman-2-yl)ethanol (6a). To a two-necked $10-\mathrm{mL}$ flask were sequentially added $(0.81 \mathrm{~g}, 4.3 \mathrm{mmol})$ of anhydrous tin(II) chloride in Glove Box and a solution of $3 \mathrm{a}(0.15 \mathrm{~g}, 0.43 \mathrm{mmol})$ in $5.4 \mathrm{~mL}$ of EtOH containing (4.3 mmol HCl, prepared from $4.3 \mathrm{mmol}$ of acetic chloride at $0{ }^{\circ} \mathrm{C}$ ) under nitrogen atmosphere. The reaction mixture was heated to reflux for $4 \mathrm{~h}$. It was then cooled to $0{ }^{\circ} \mathrm{C}$, quenched with sat. $\mathrm{NH}_{4} \mathrm{Cl}$ and basified with $\mathrm{NH}_{3} \cdot \mathrm{H}_{2} \mathrm{O}$. Dichloromethane was added and vigorously stirred for $10 \mathrm{~min}$. The white suspension was suction filtrated over Celit. The aqueous phase was extracted with $\mathrm{CH}_{2} \mathrm{Cl}_{2}$ and the combined organic phase was dried over $\mathrm{Na}_{2} \mathrm{SO}_{4}$. Then the crude product was purified by flash silica gel chromatography to afford the product $\mathbf{6 a}$ as white solid in $88 \%$ yield. ${ }^{\mathbf{1}} \mathbf{H} \mathbf{~ N M R}$ (600 $\left.\mathrm{MHz}, \mathrm{CDCl}_{3}\right) \delta(\mathrm{ppm}) 8.08(\mathrm{~d}, J=8.3 \mathrm{~Hz}, 1 \mathrm{H}), 7.42-7.17(\mathrm{~m}, 3 \mathrm{H}), 6.99(\mathrm{~d}, J=7.7 \mathrm{~Hz}, 1 \mathrm{H}), 6.78$ $(\mathrm{t}, J=7.4 \mathrm{~Hz}, 1 \mathrm{H}), 6.54(\mathrm{~s}, 1 \mathrm{H}), 6.31(\mathrm{~s}, 1 \mathrm{H}), 4.34(\mathrm{~s}, 1 \mathrm{H}), 4.02-3.84(\mathrm{~m}, 2 \mathrm{H}), 2.65(\mathrm{~s}, 3 \mathrm{H}), 2.28-$ $2.24(\mathrm{~m}, 1 \mathrm{H}), 2.03-1.99(\mathrm{~m}, 1 \mathrm{H}), 0.91(\mathrm{~s}, 3 \mathrm{H}) ;{ }^{13} \mathbf{C} \mathbf{N M R}\left(150 \mathrm{MHz}, \mathrm{CDCl}_{3}\right) \delta$ (ppm) 154.41, 146.07, 129.75, 128.27, 128.24, 127.02, 123.84, 121.21, 119.96, 117.11, 81.21, 65.69, 59.12, 53.18, 32.20, 17.23; HRMS (MALDI); Calcd for $\mathrm{C}_{18} \mathrm{H}_{20} \mathrm{~N}_{4} \mathrm{O}_{2}$ [M+Na]: 347.1484; Found: 347.1473. 
6. Copies of ${ }^{1} \mathrm{H}$ NMR and ${ }^{13} \mathrm{C}$ NMR Spectrums

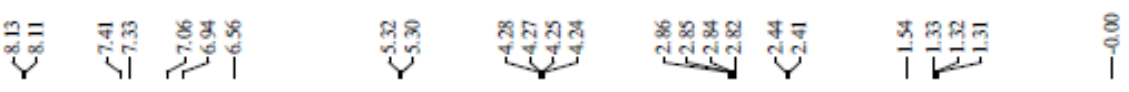<smiles></smiles>

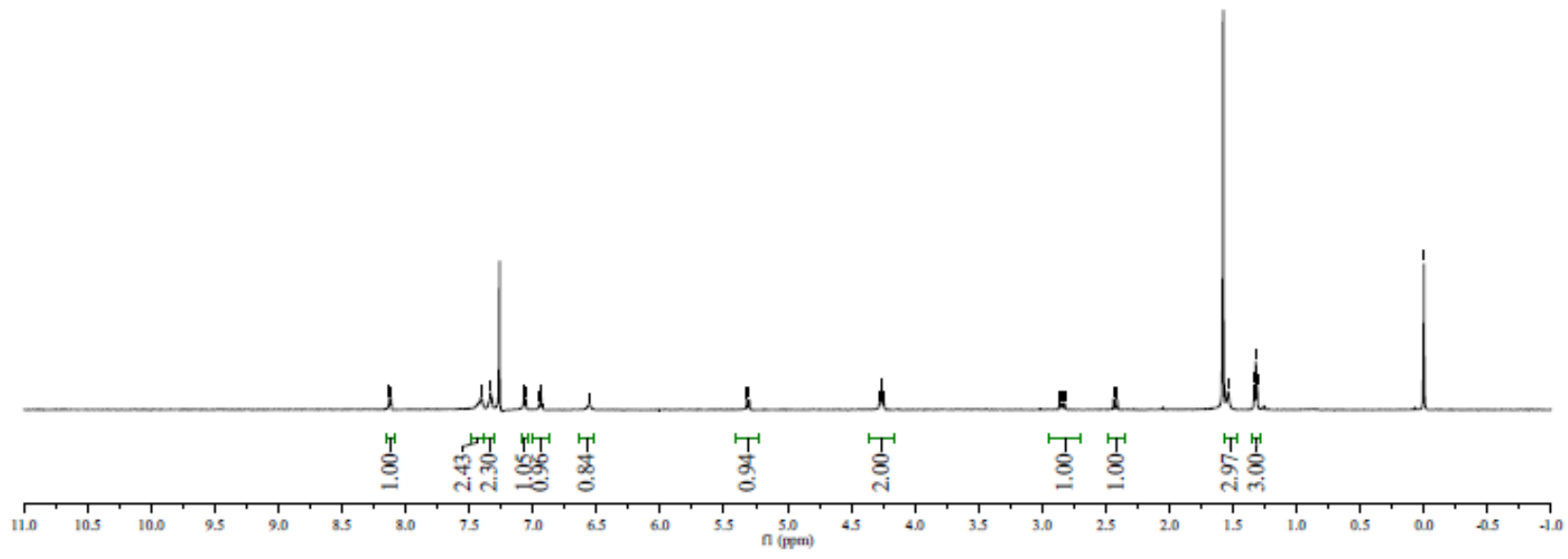

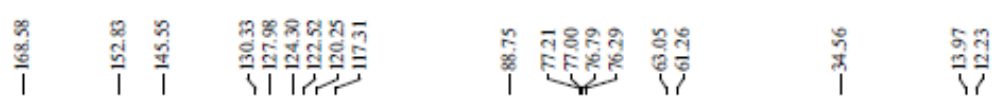
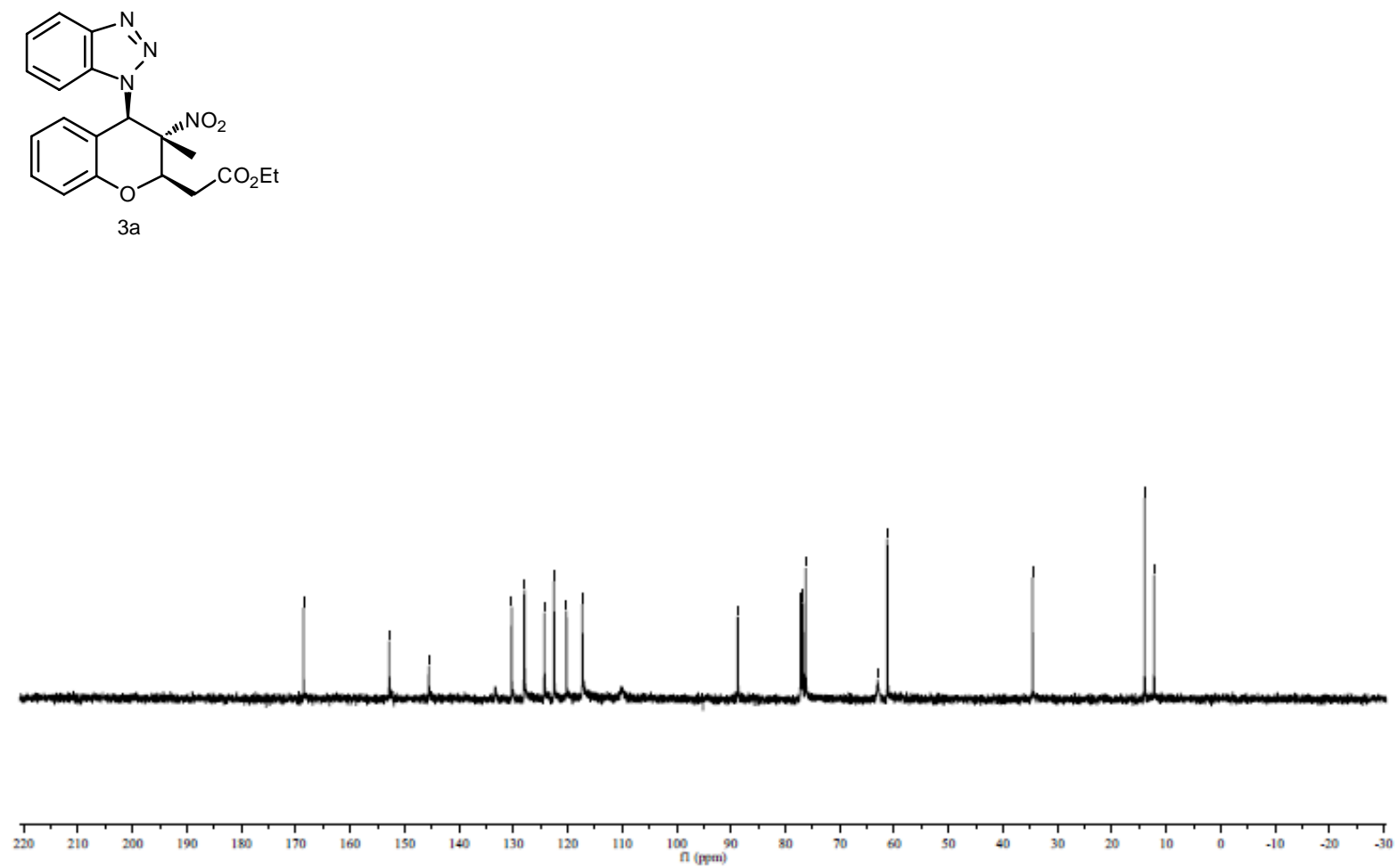


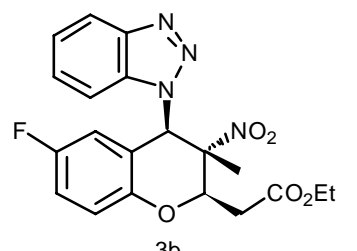

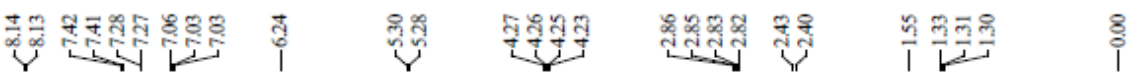
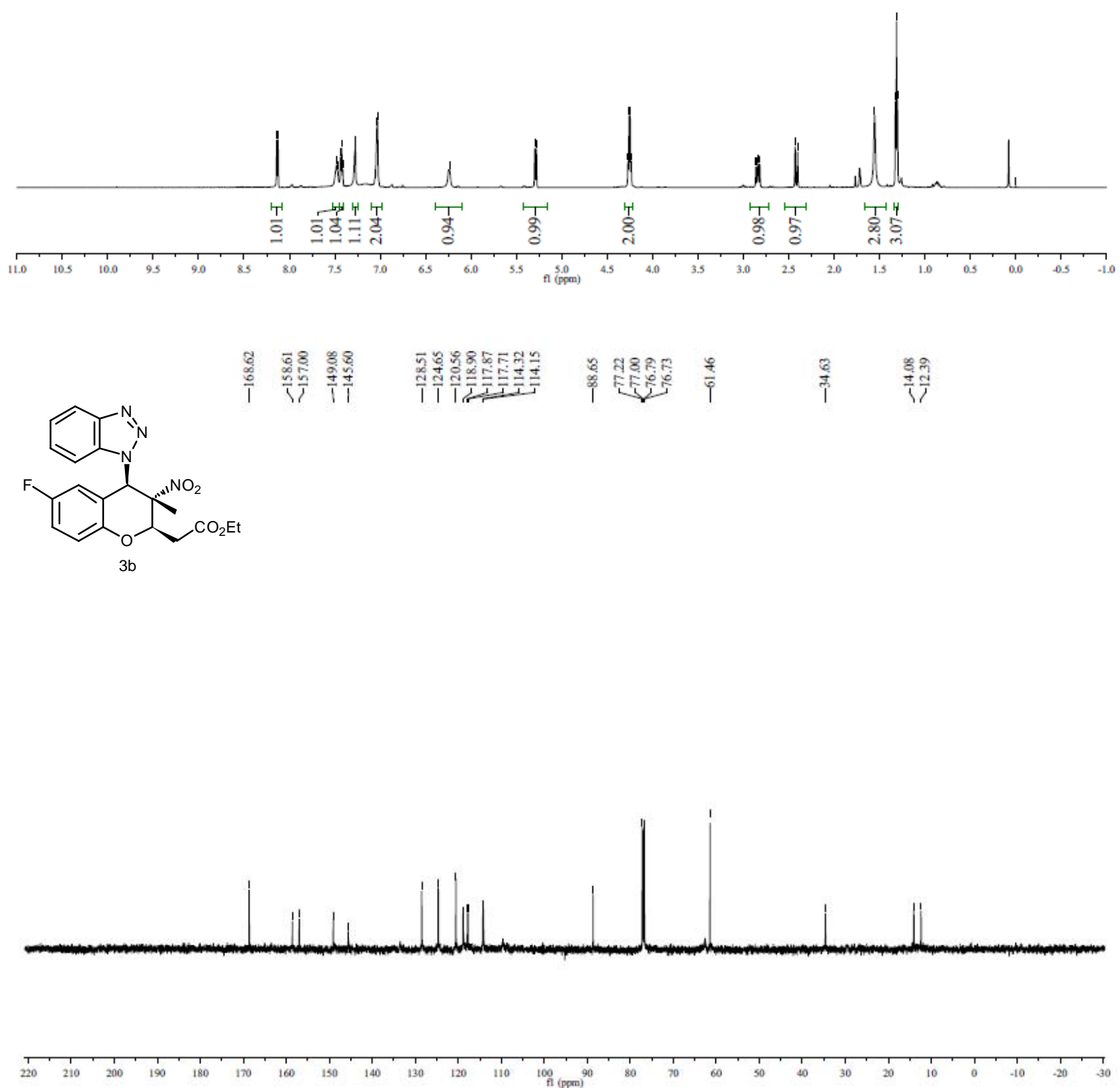
<smiles>CCOCC1Oc2ccc(Cl)cc2[C@@H](n2nnc3ccccc32)[C@]1(C)O</smiles>
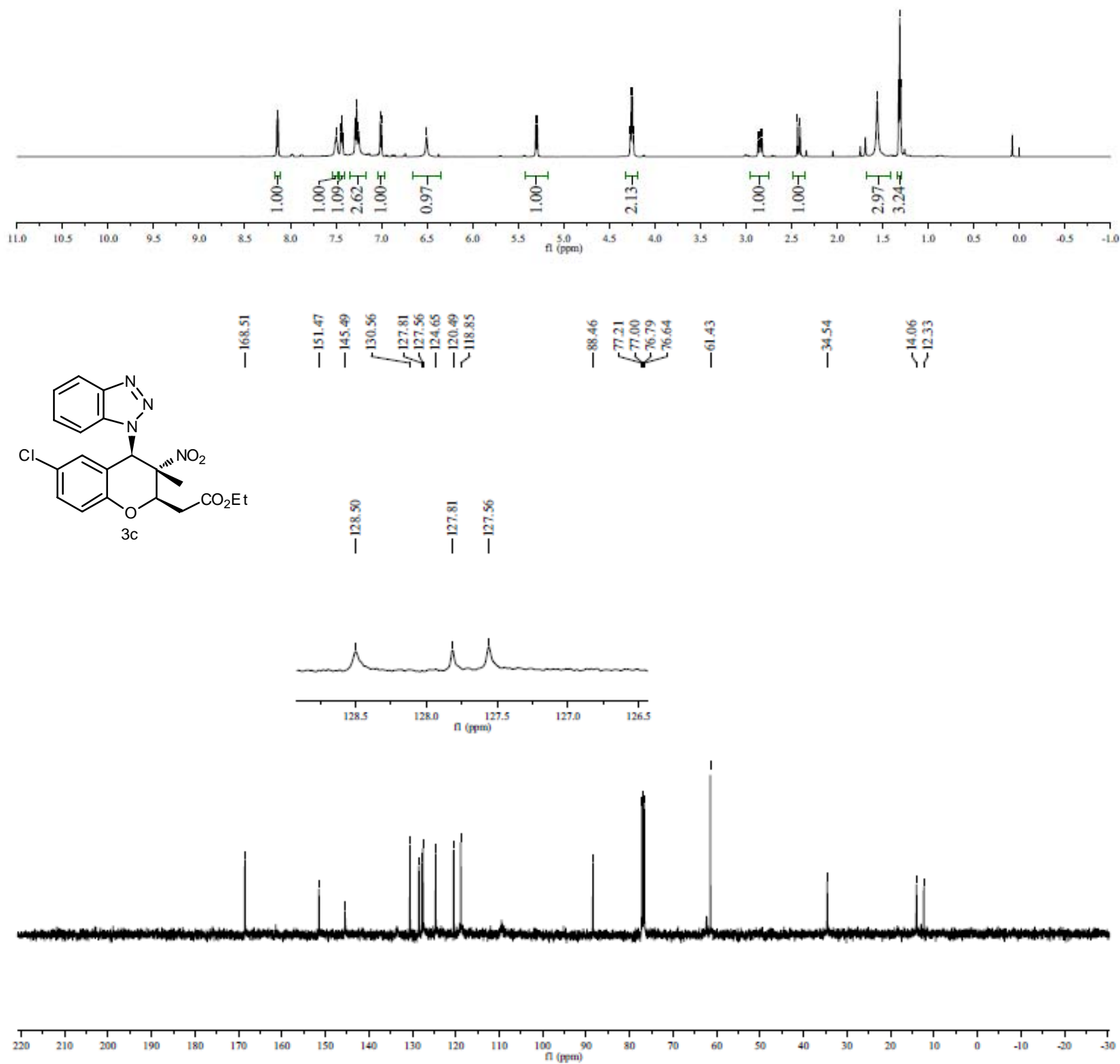


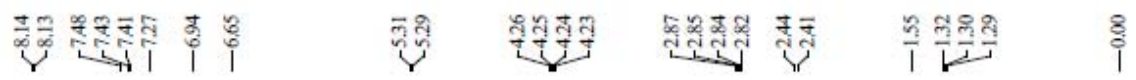

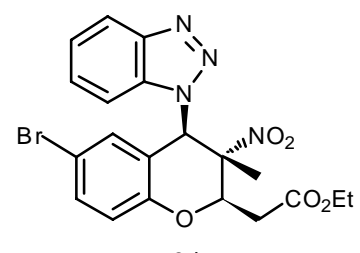

$3 d$
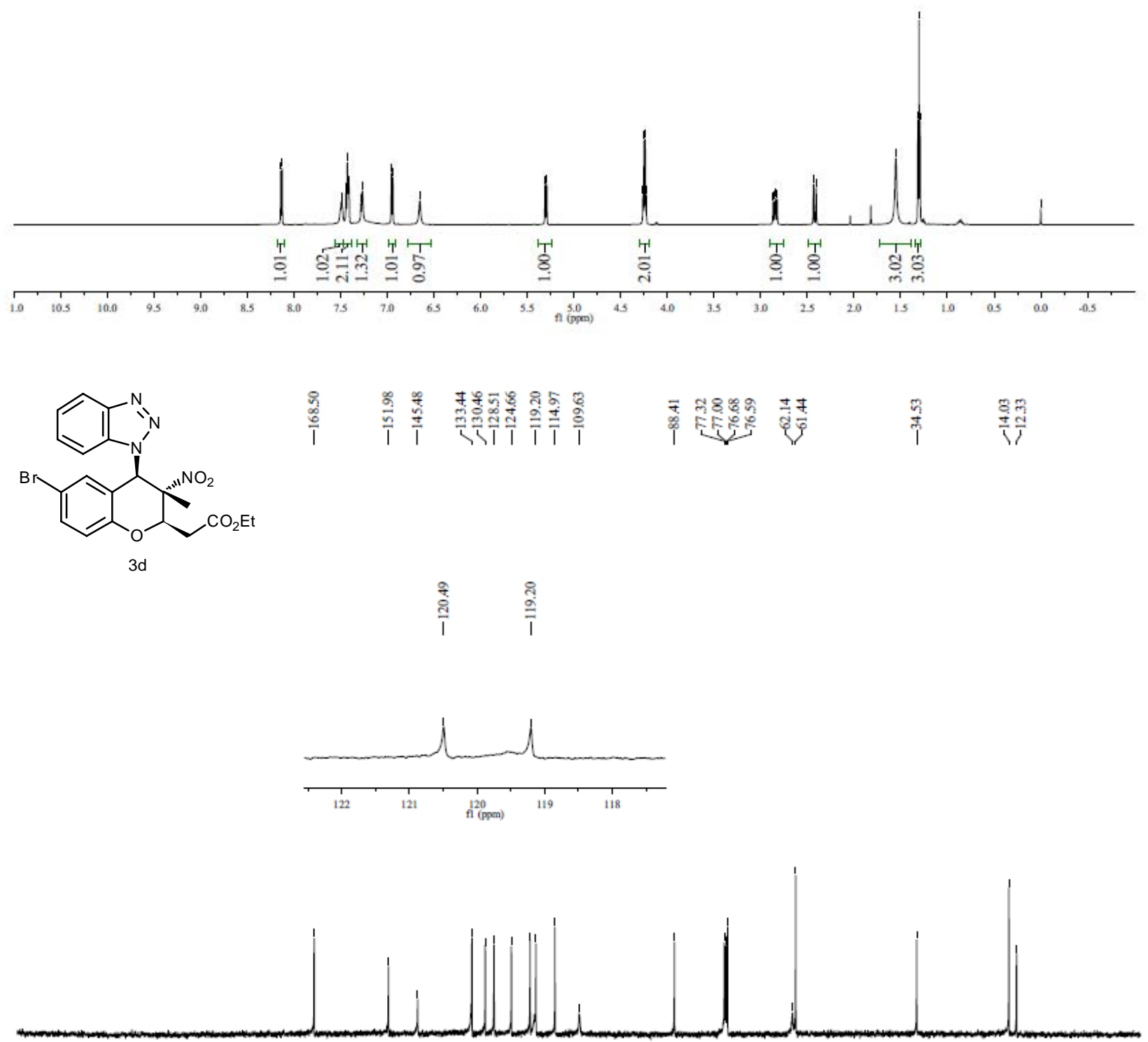

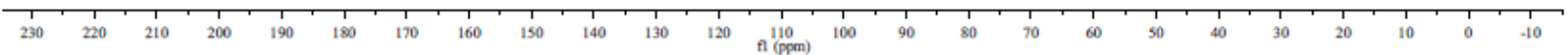



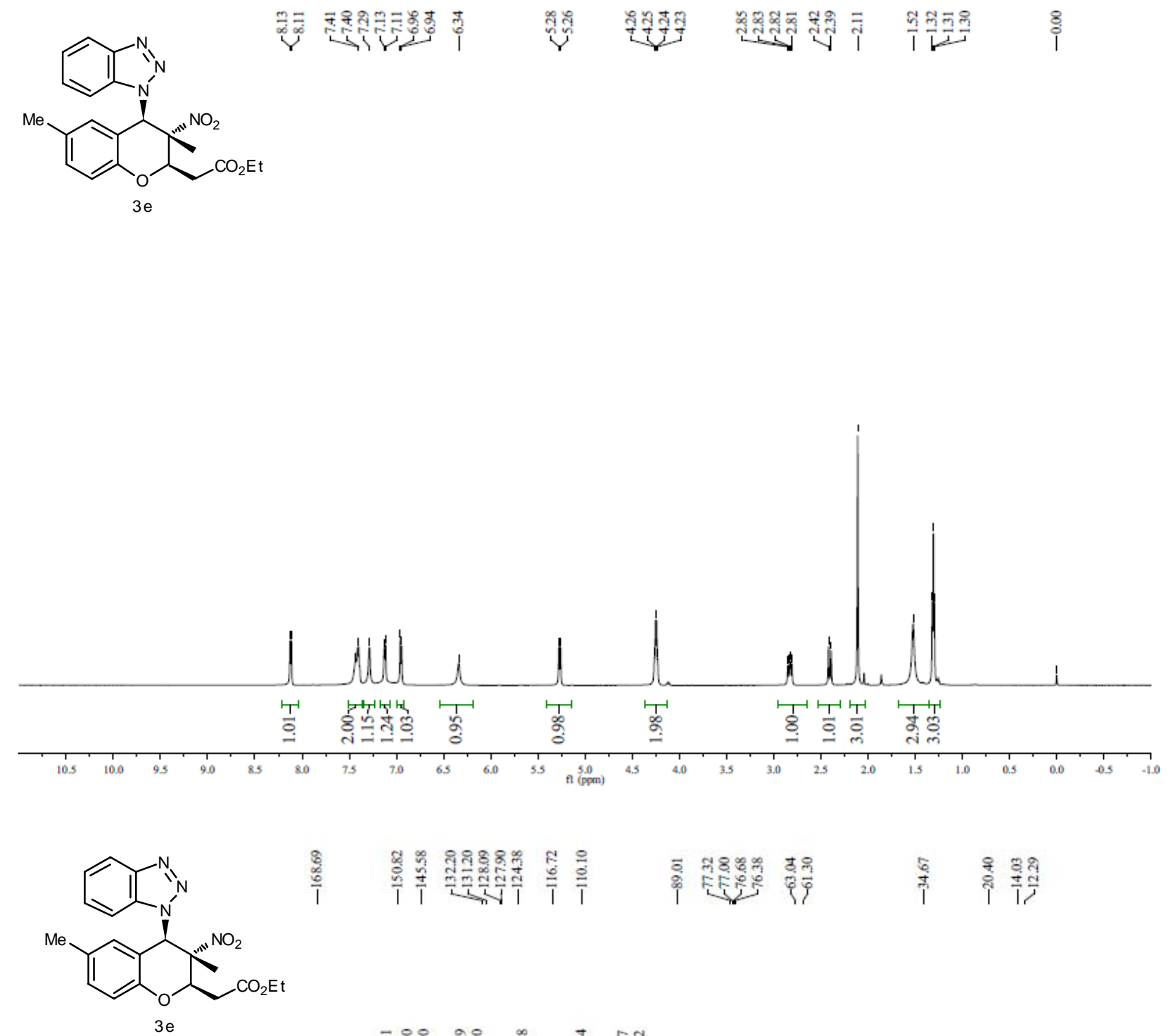

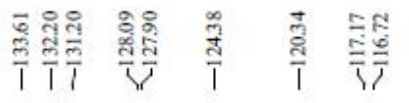
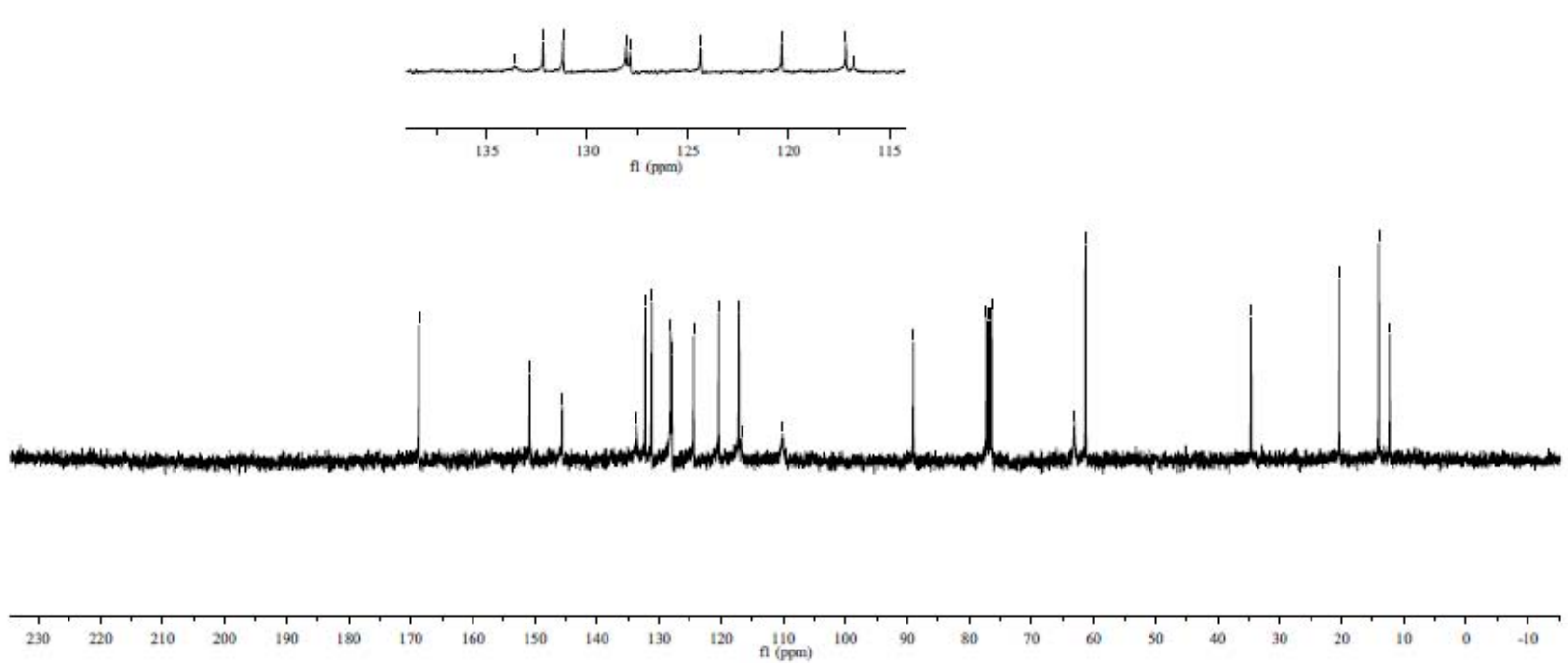


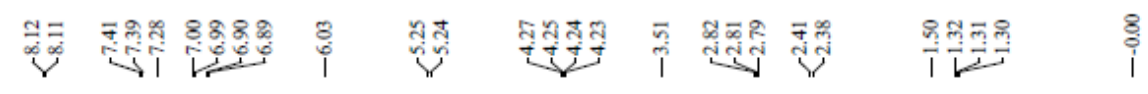<smiles>CCOCC1Oc2ccc(OC)cc2C(n2nnc3ccccc32)[C@]1(O)O[Na]</smiles>

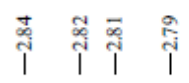
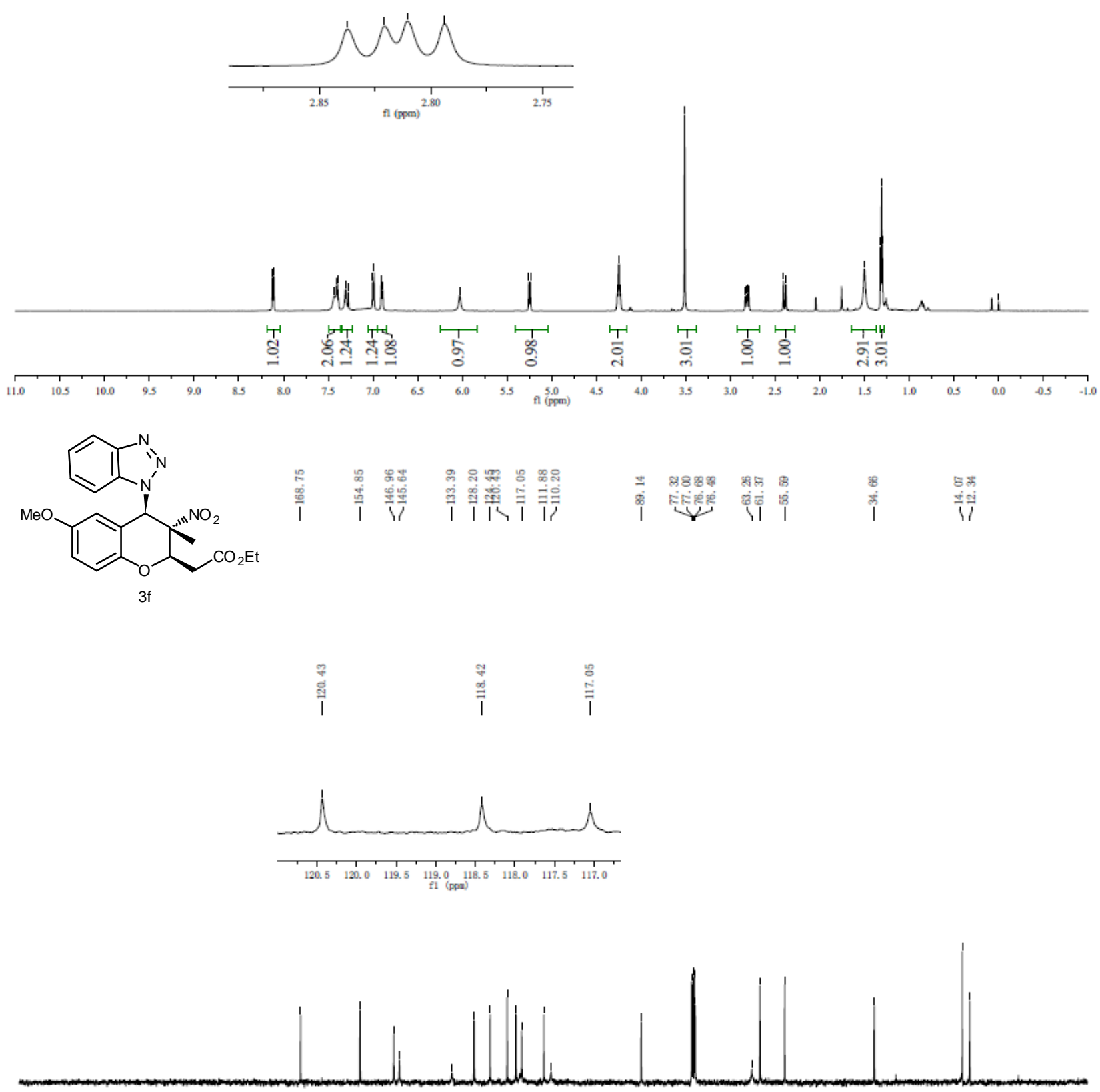

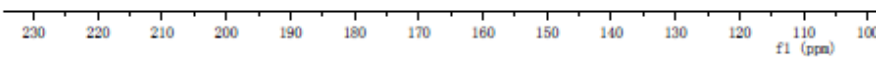


<smiles>CCOC(=O)CC1Oc2cc(OC)ccc2C(n2nnc3ccccc32)C1([N+](=O)[O-])[N+](=O)[O-]</smiles>

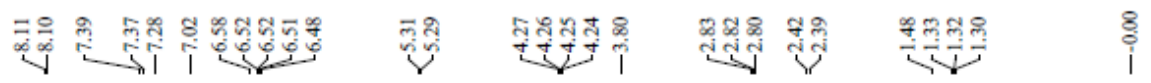

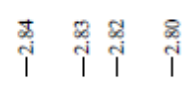
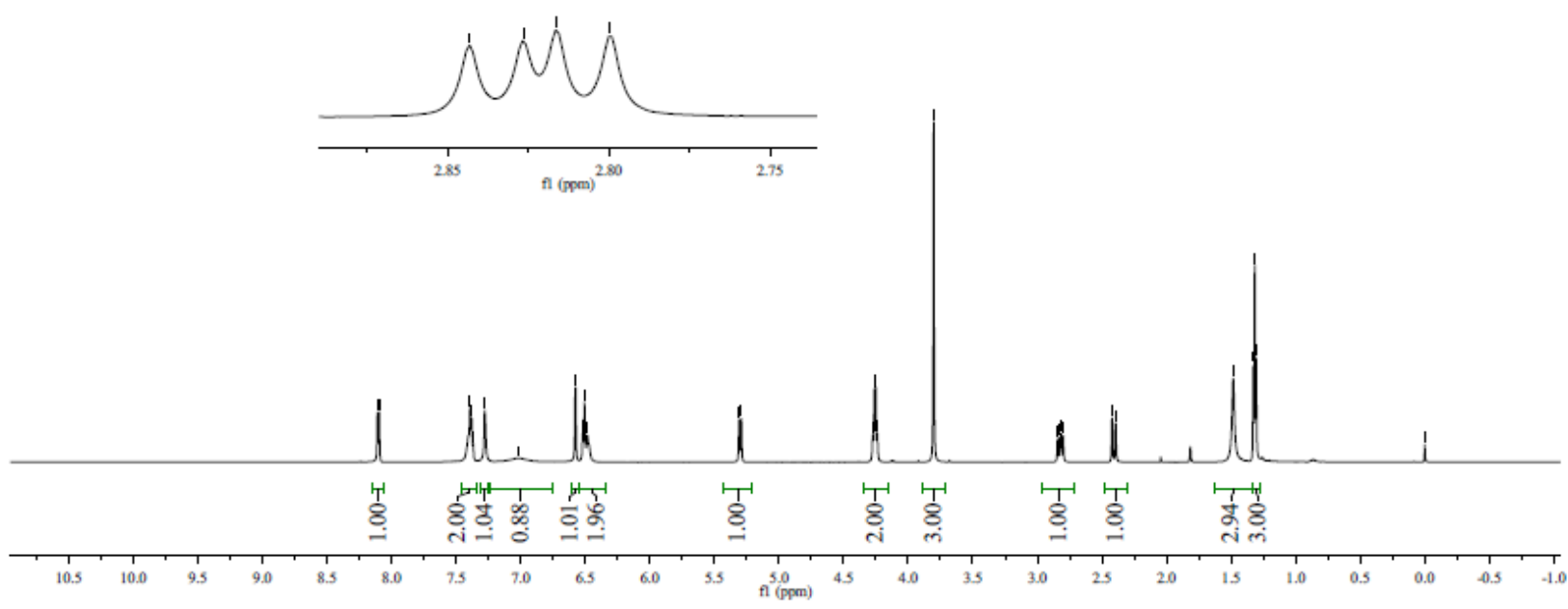

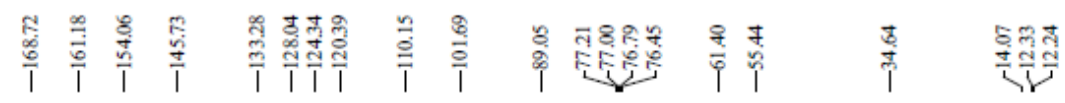<smiles></smiles>

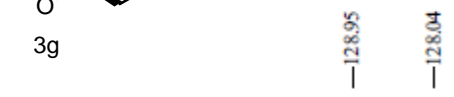
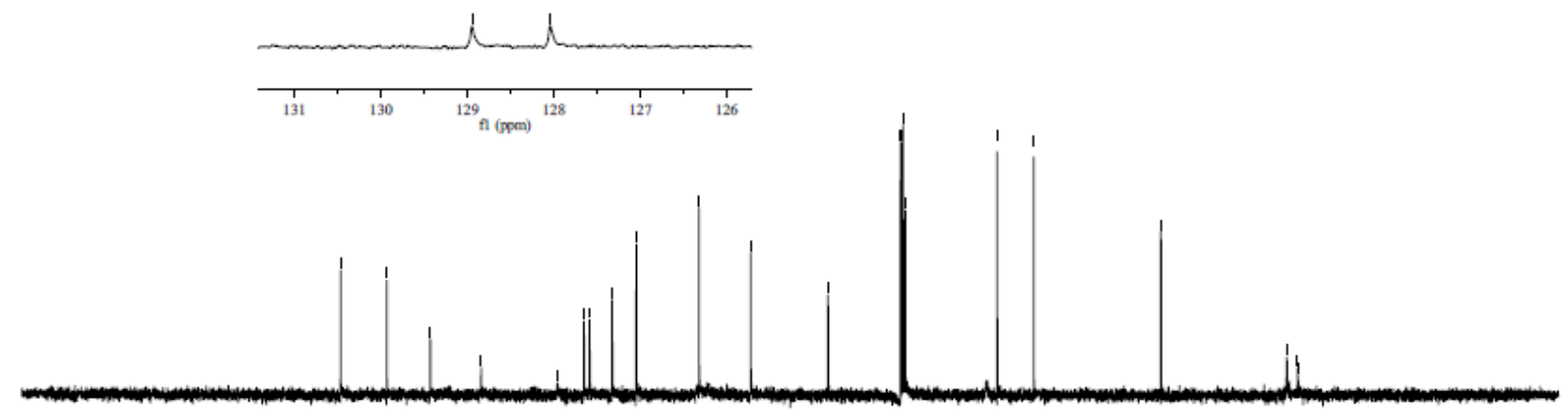

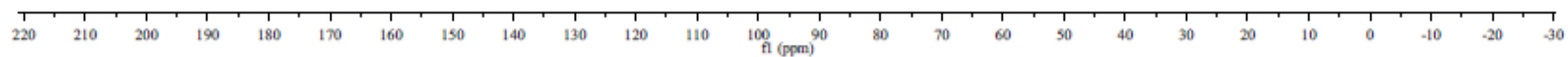




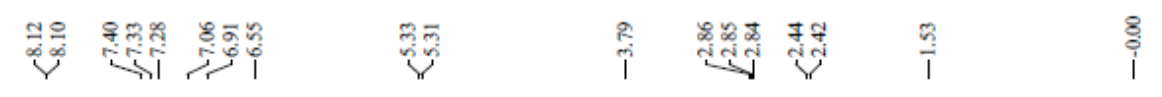

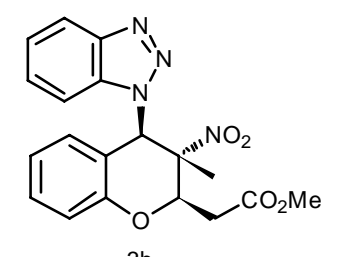

$3 \mathrm{~h}$

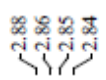
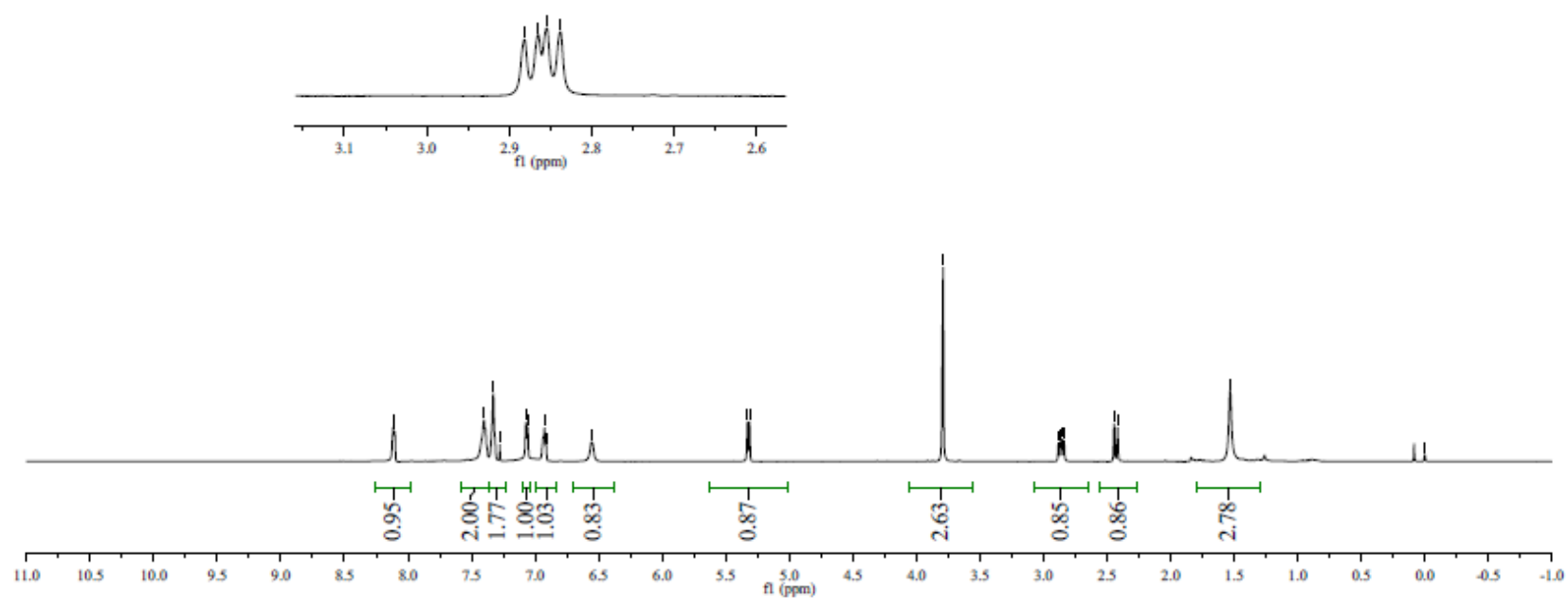

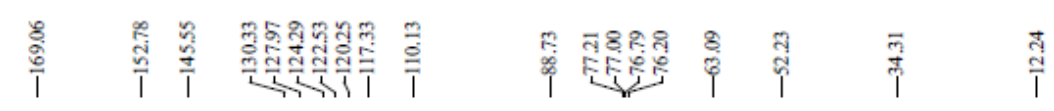<smiles>CC(=O)CC1Oc2ccccc2[C@H](n2nnc3ccccc32)[C@]1(C)O</smiles>

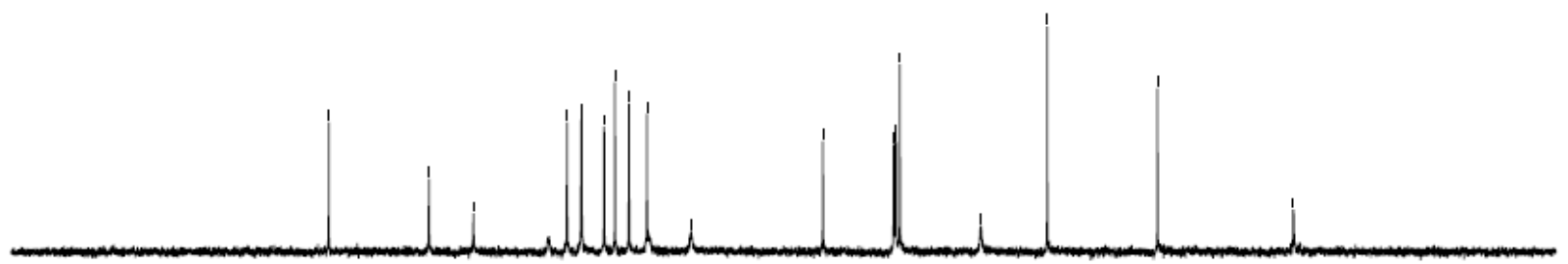

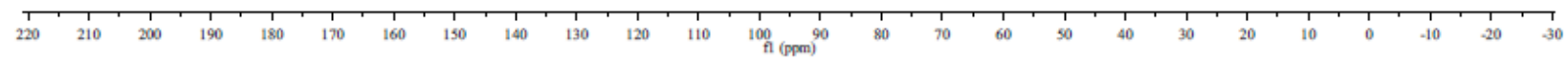




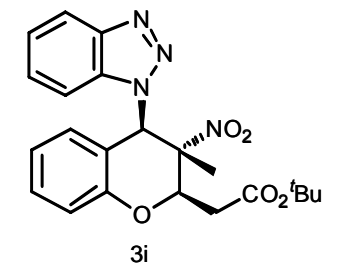

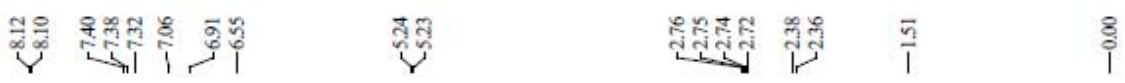
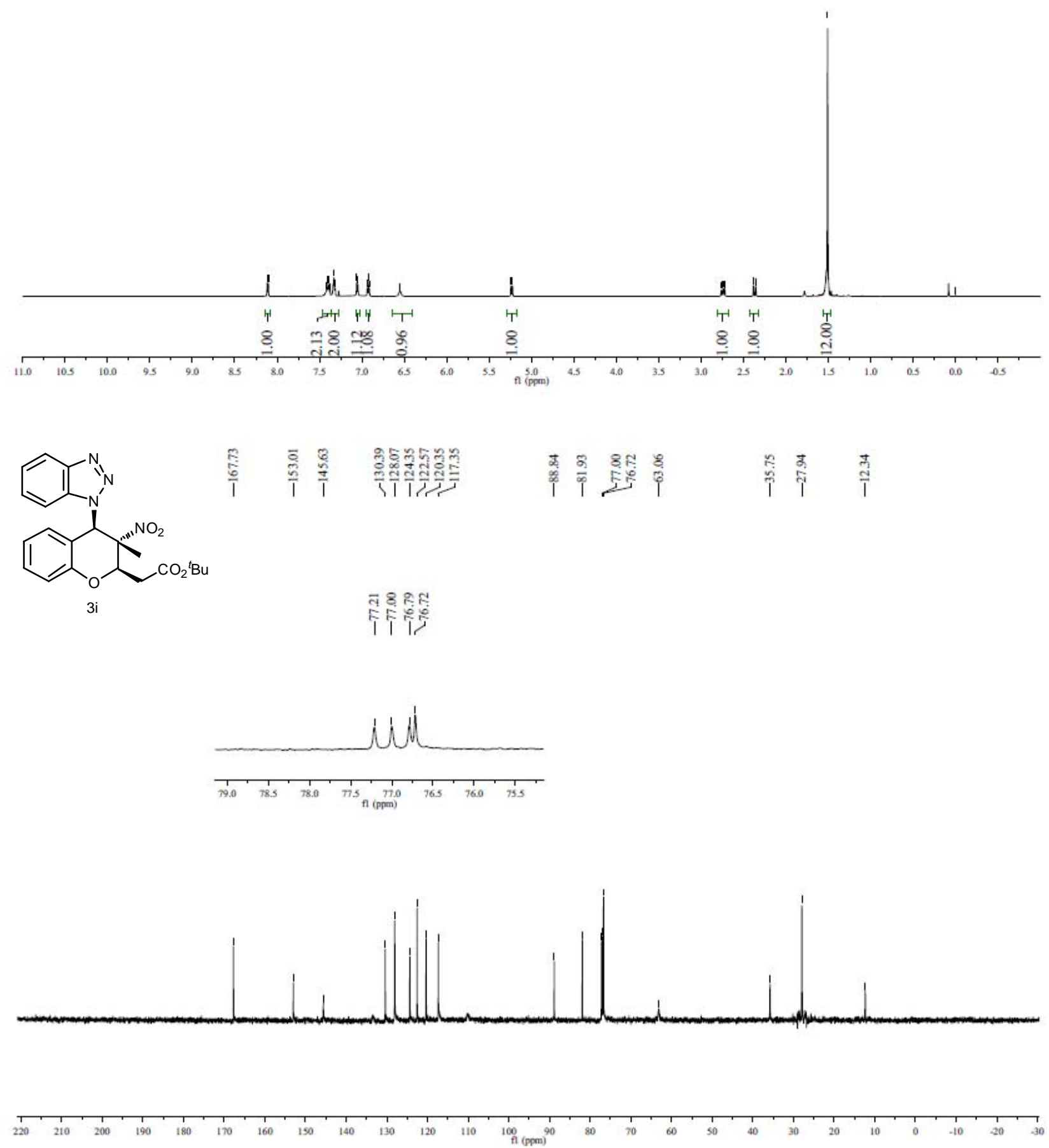


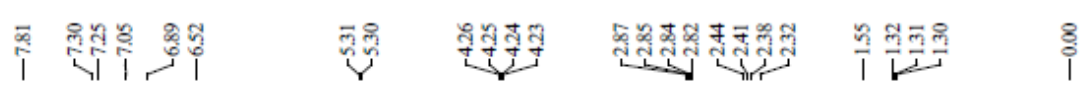
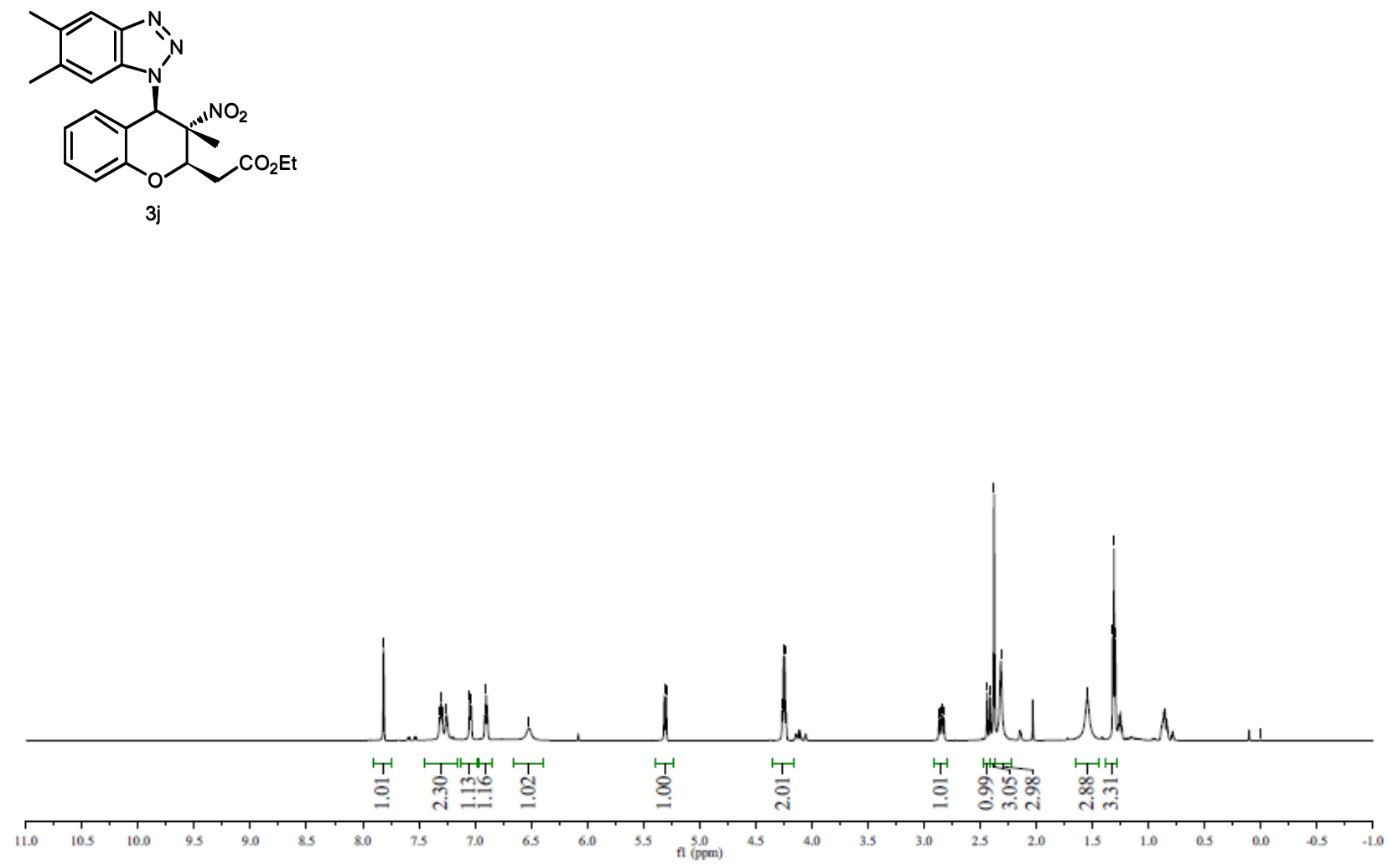

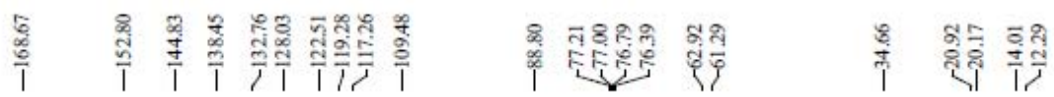<smiles>CCOC(=O)CC1Oc2ccccc2C1n1nnc2cc(C)c(C)cc21</smiles>

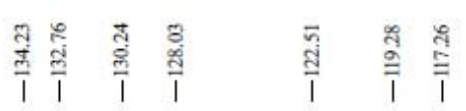
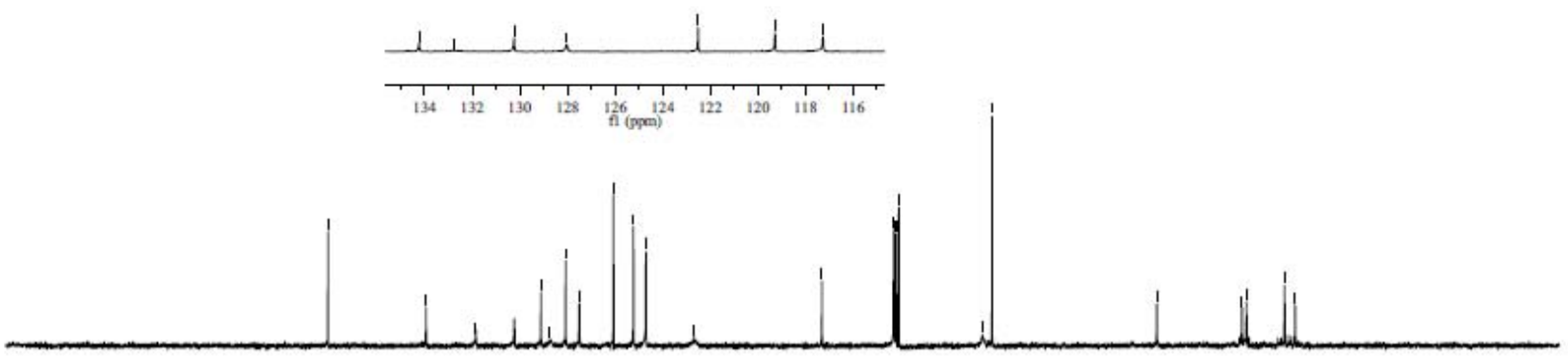

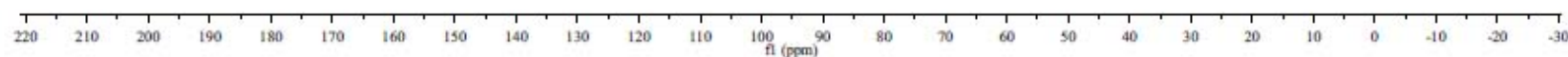




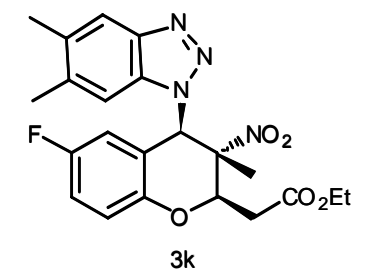

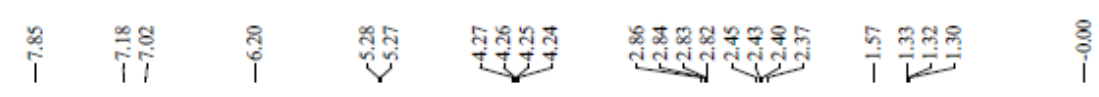
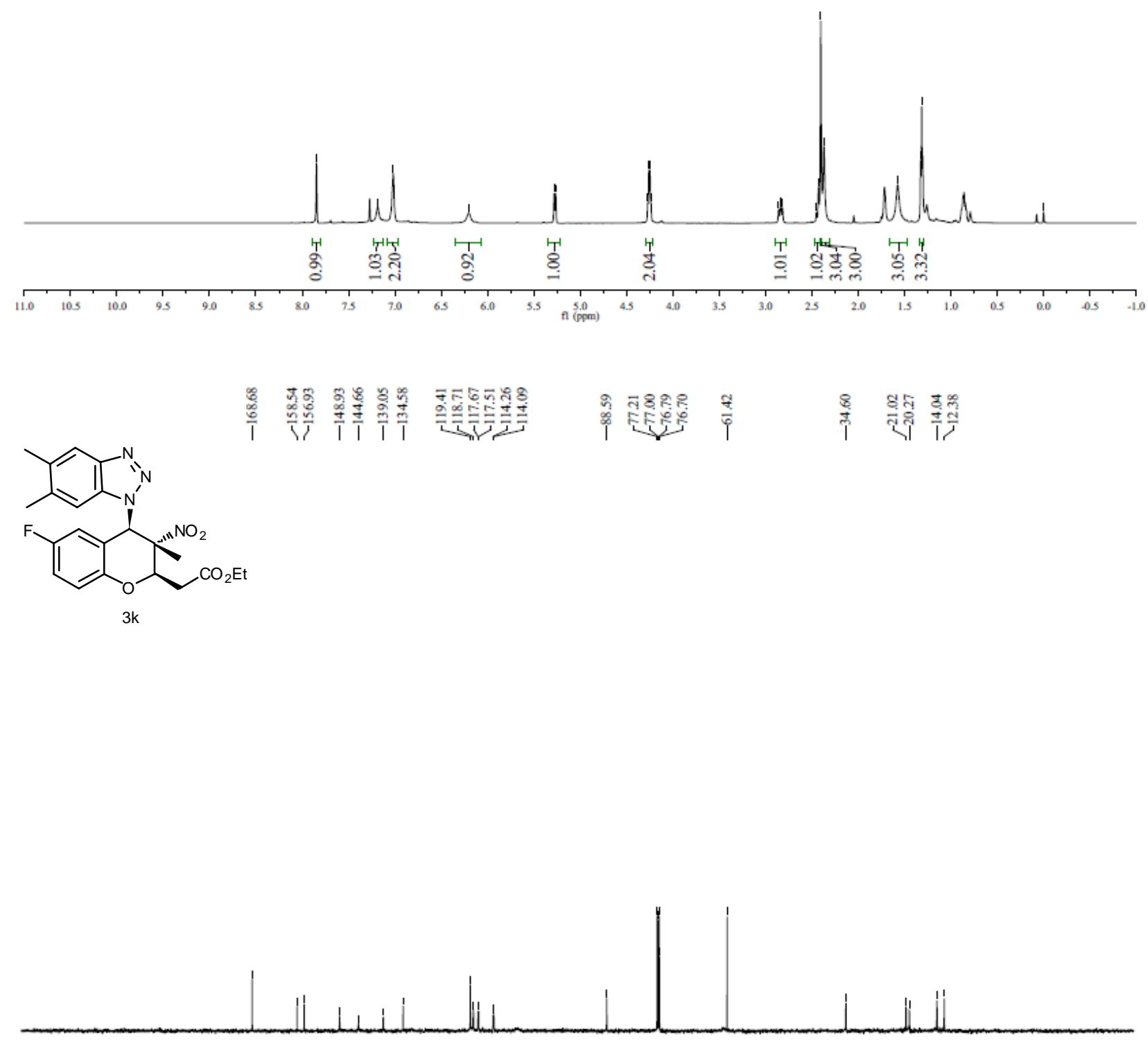

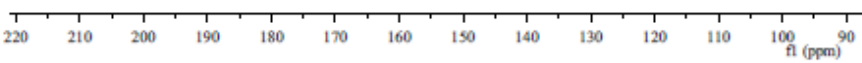




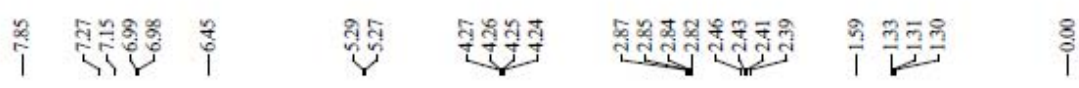<smiles>CCOC(=O)CC1Oc2ccc(Cl)cc2C(n2nnc3cc(C)c(C)cc32)[C@]1(C)O</smiles>

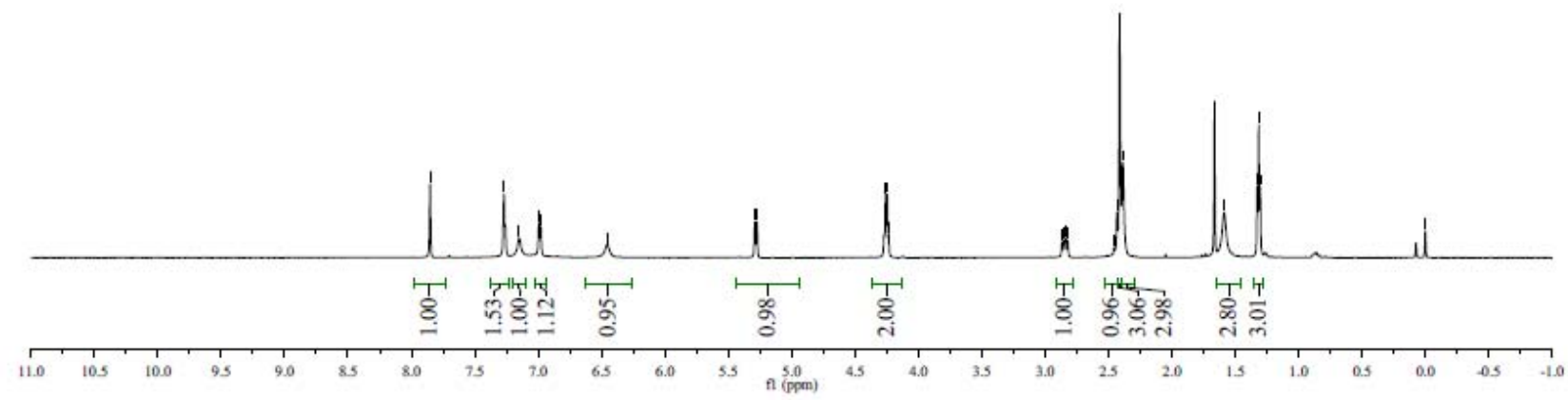

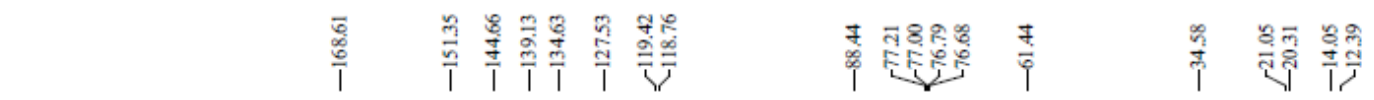<smiles>CCOCC[C@H]1Oc2ccc(Cl)cc2C(n2nnc3cc(C)c(C)cc32)[C@]1(C)O</smiles>

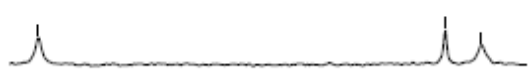

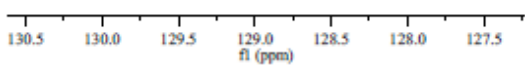

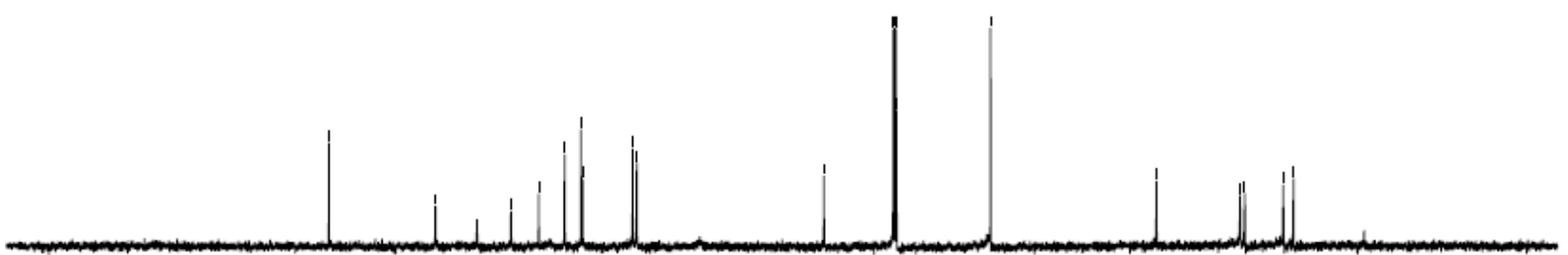

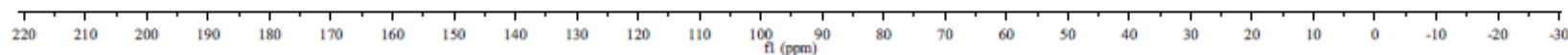




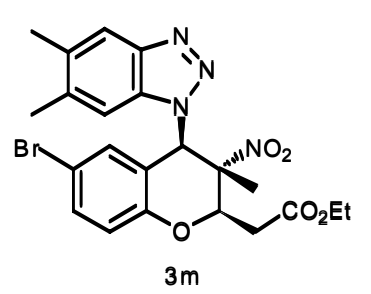

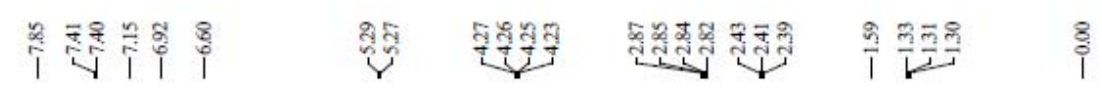
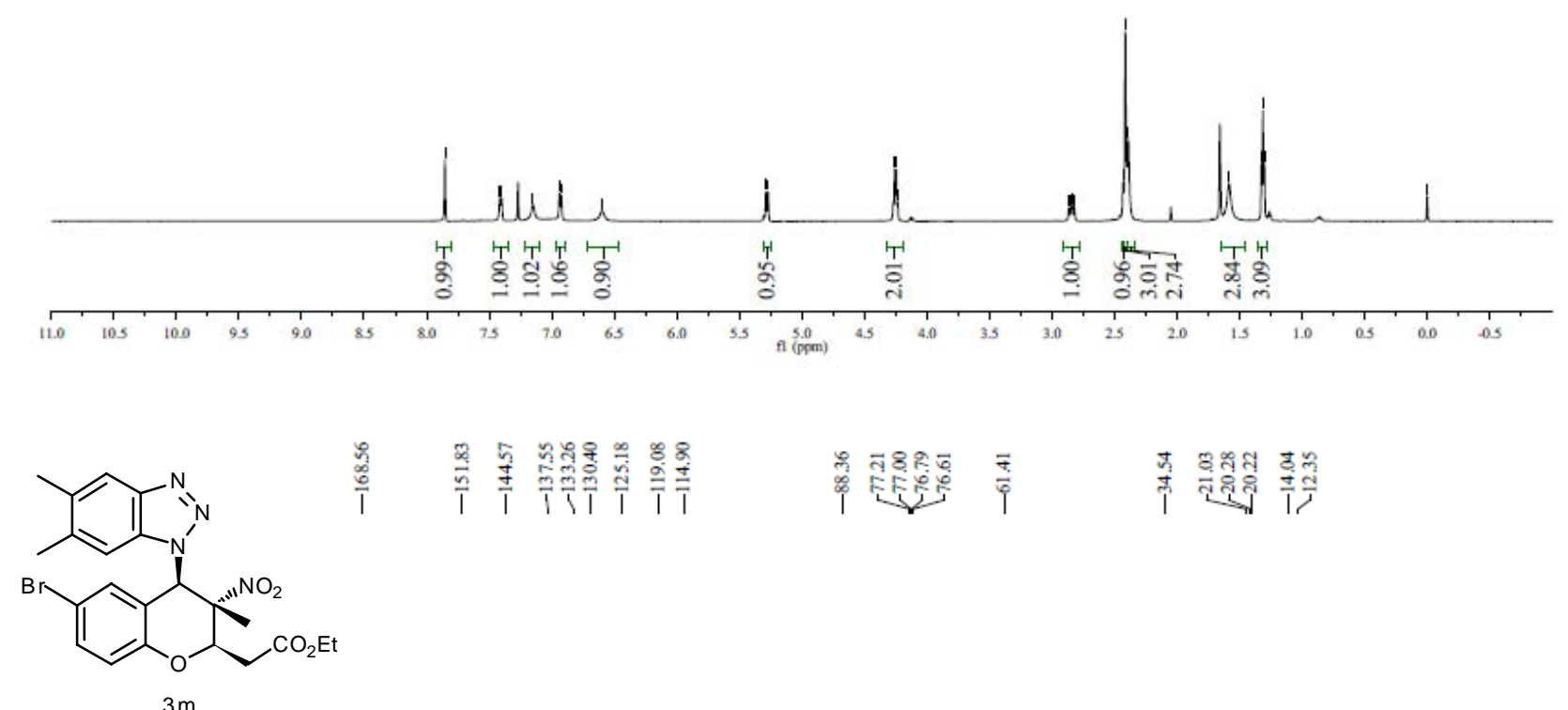

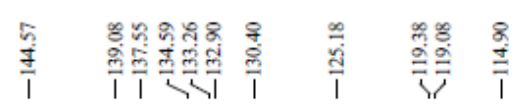
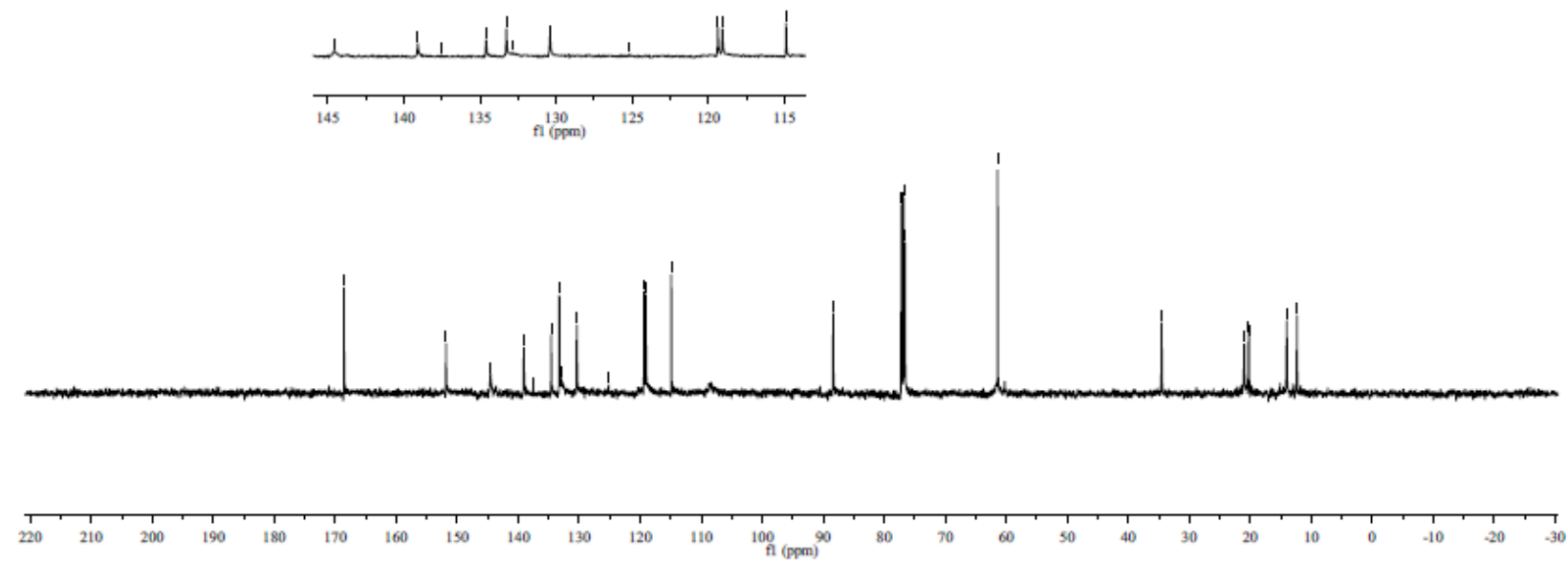
<smiles>CCOCC1Oc2cc(OC)ccc2C(n2nnc3cc(C)c(C)cc32)C1(O)O</smiles>

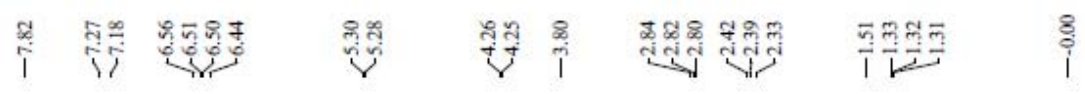

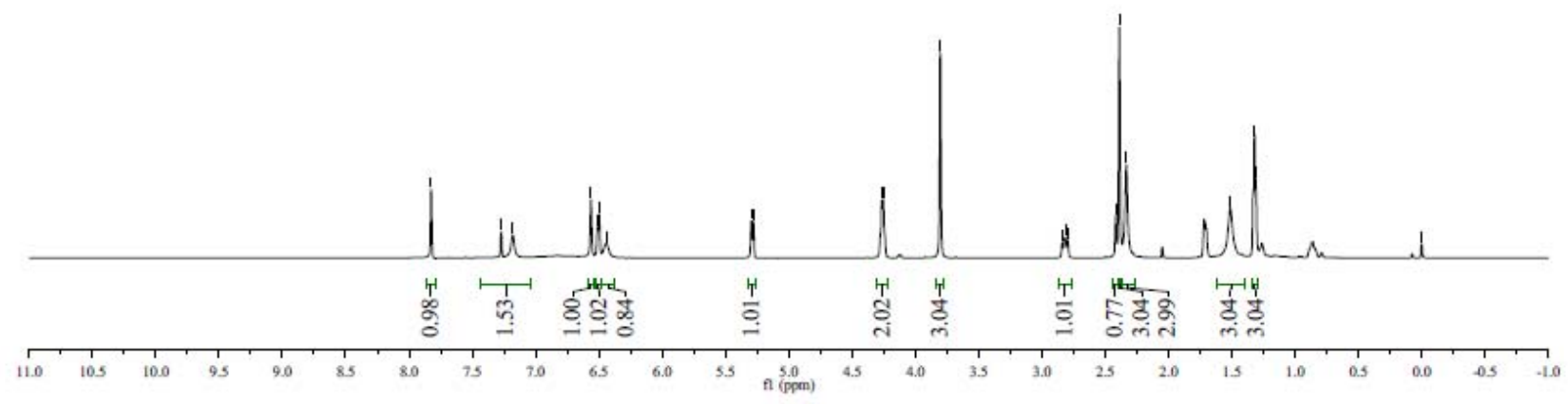

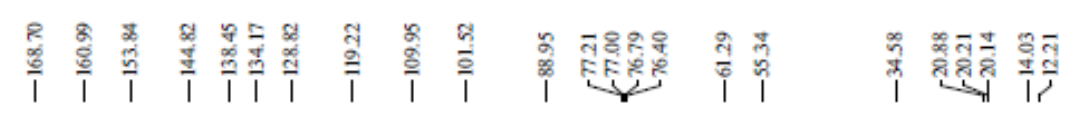<smiles>CCOCC1Oc2cc(OC)ccc2C(n2nnc3cc(C)c(C)cc32)[C@]1(O)COCC</smiles>
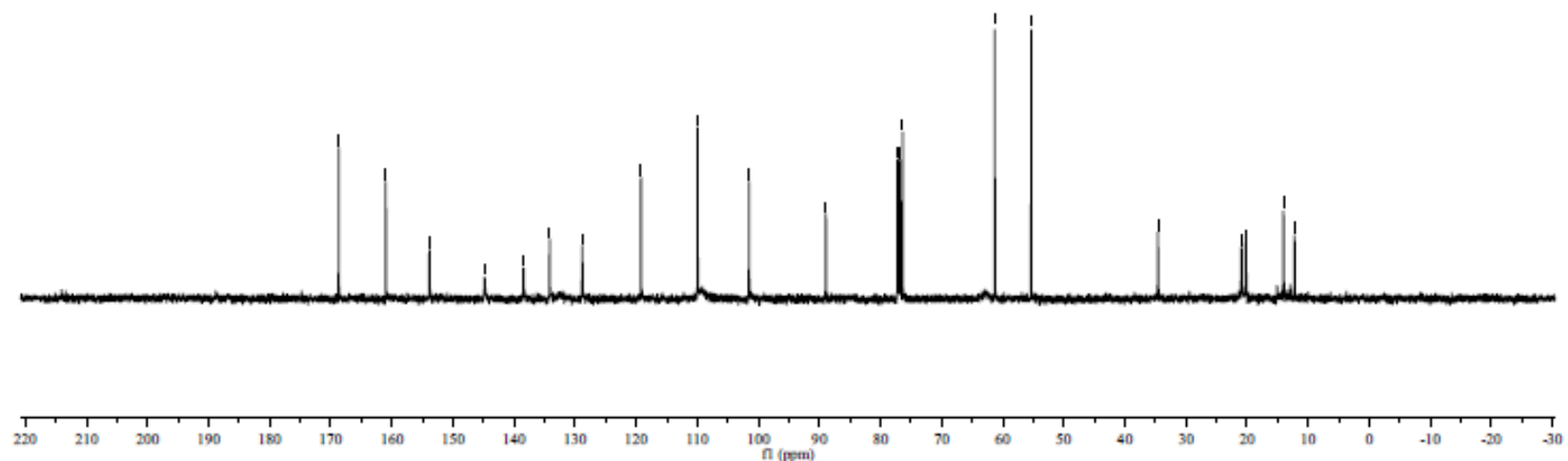


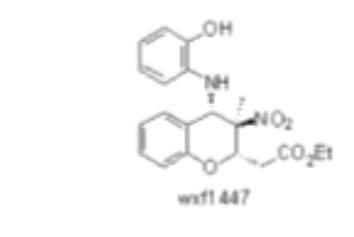

3o

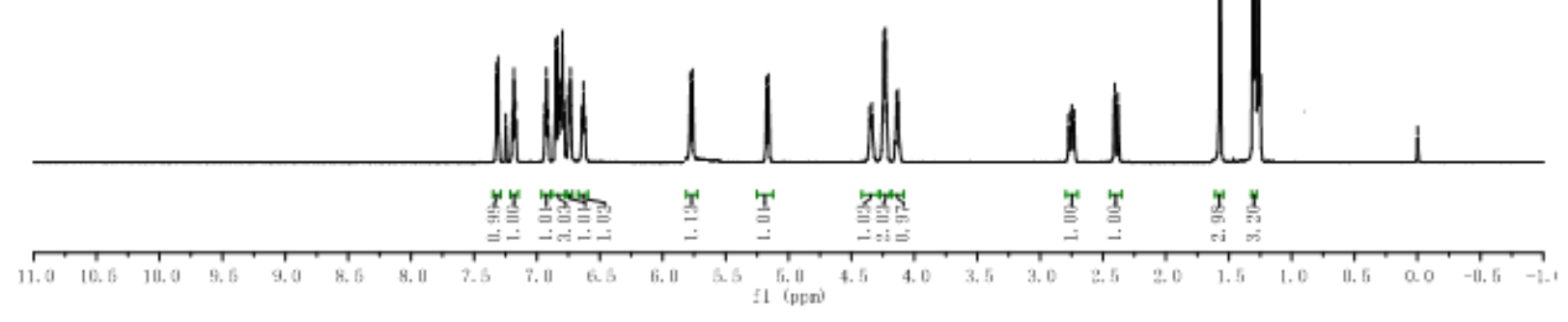

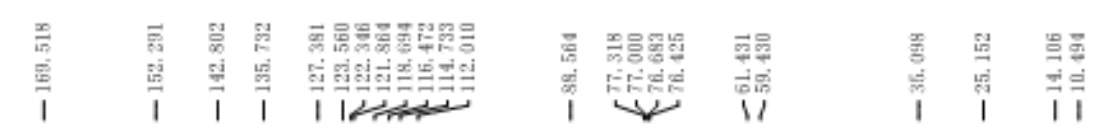

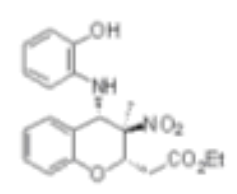

30

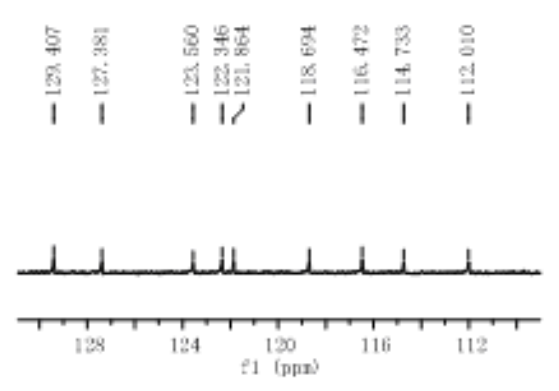

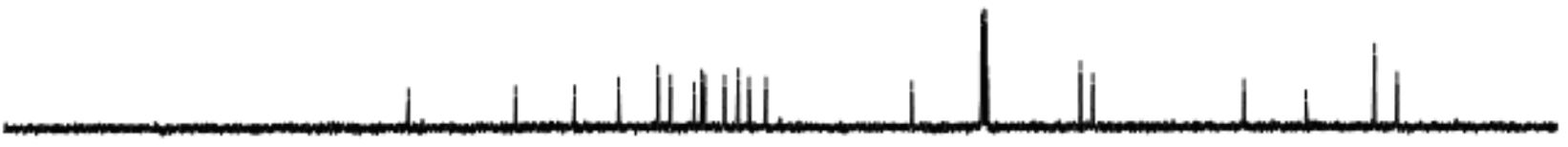

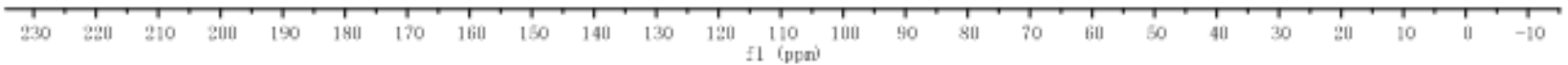




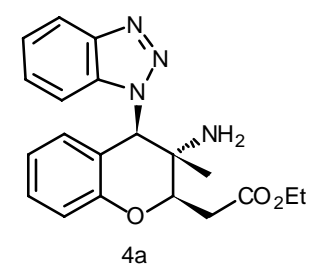

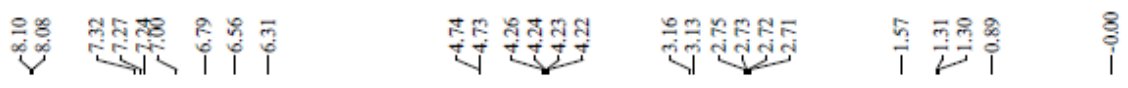
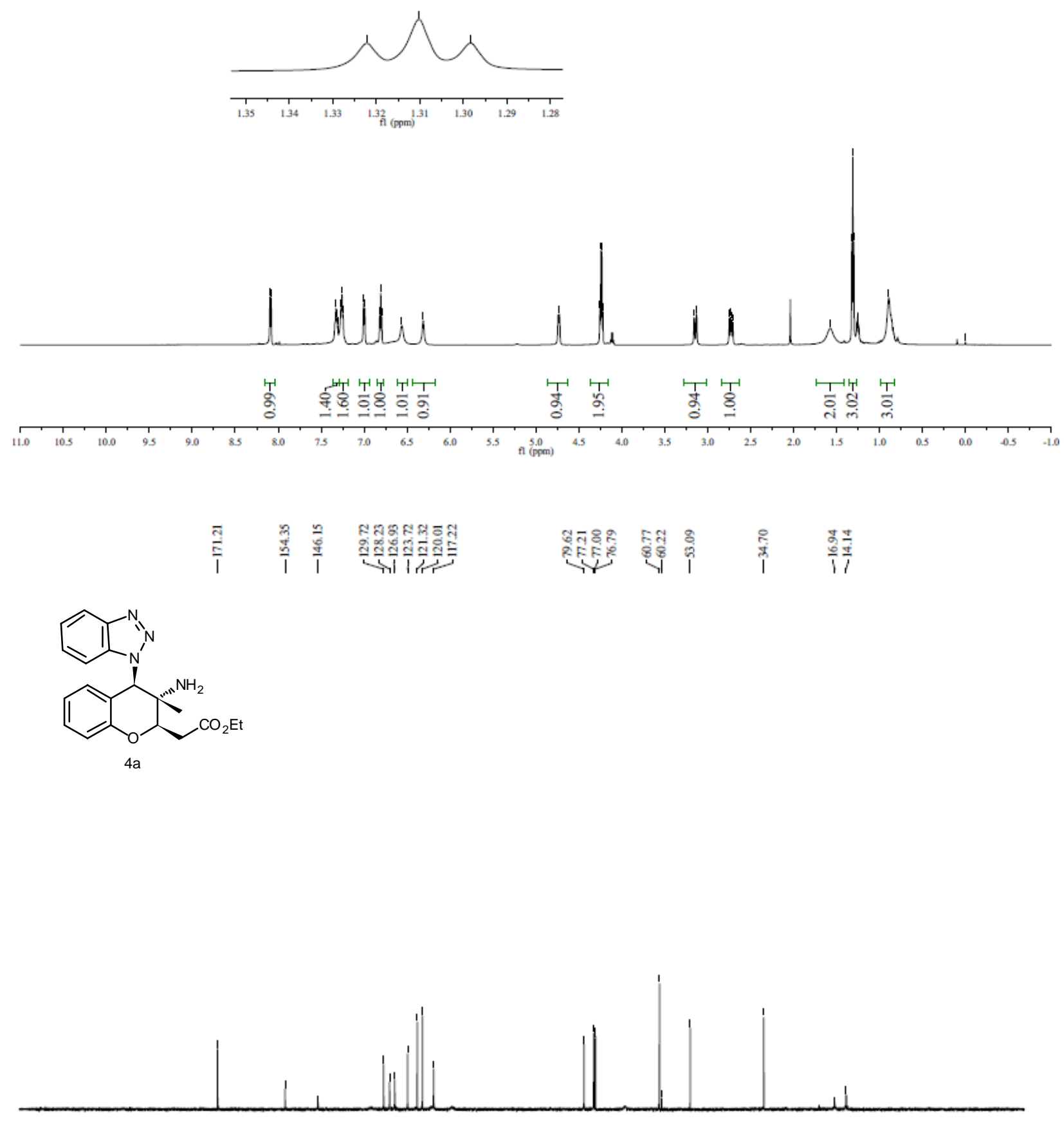

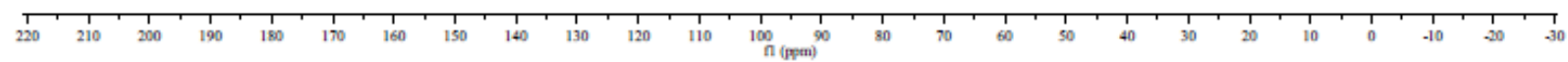




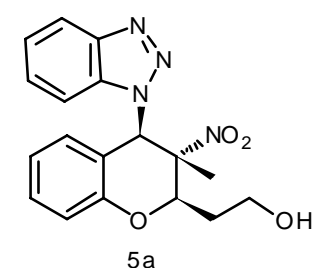

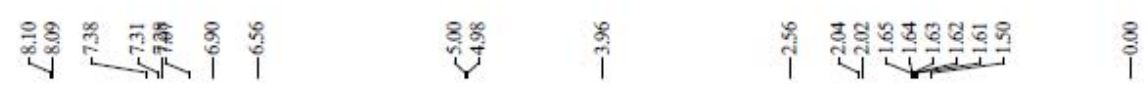
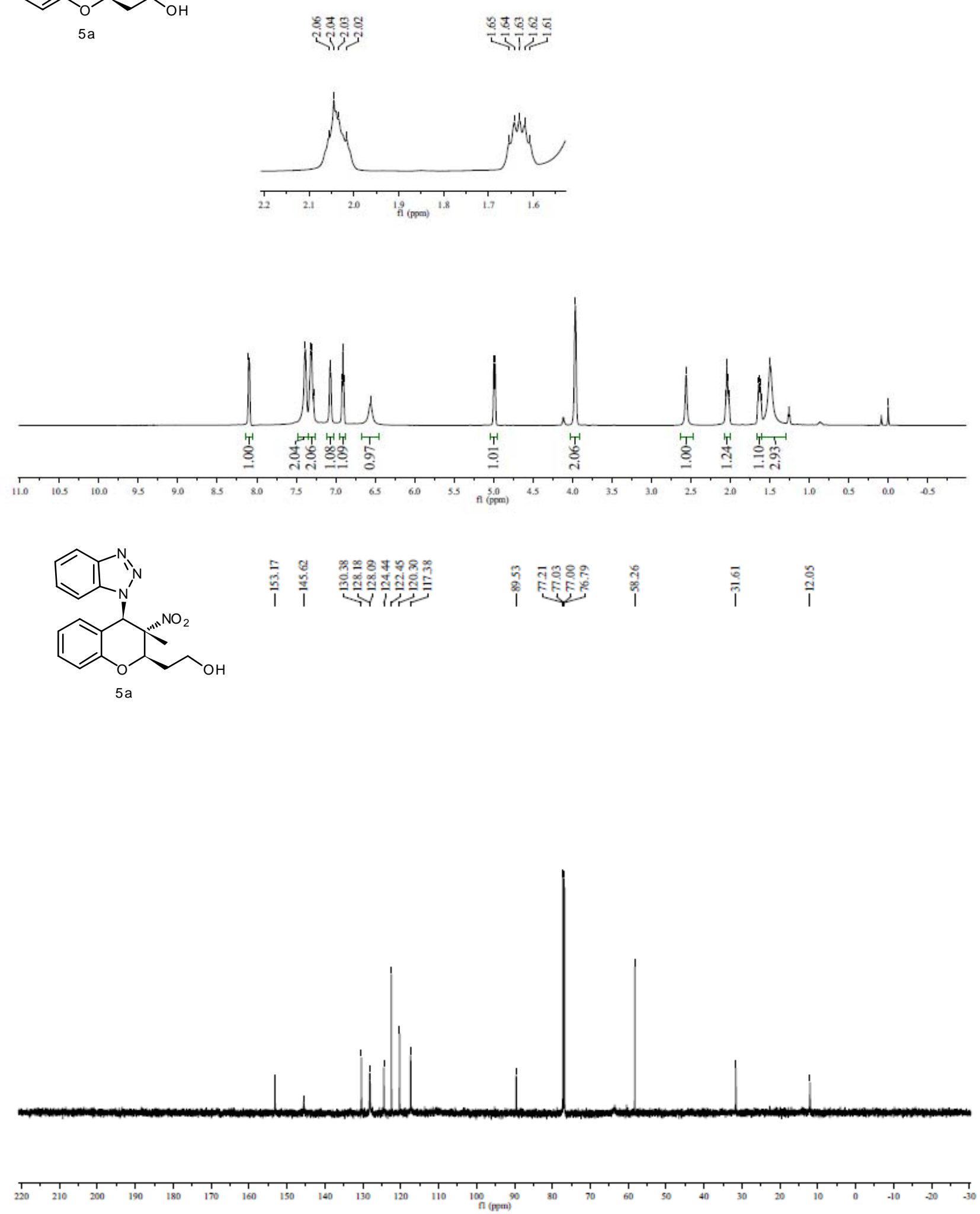

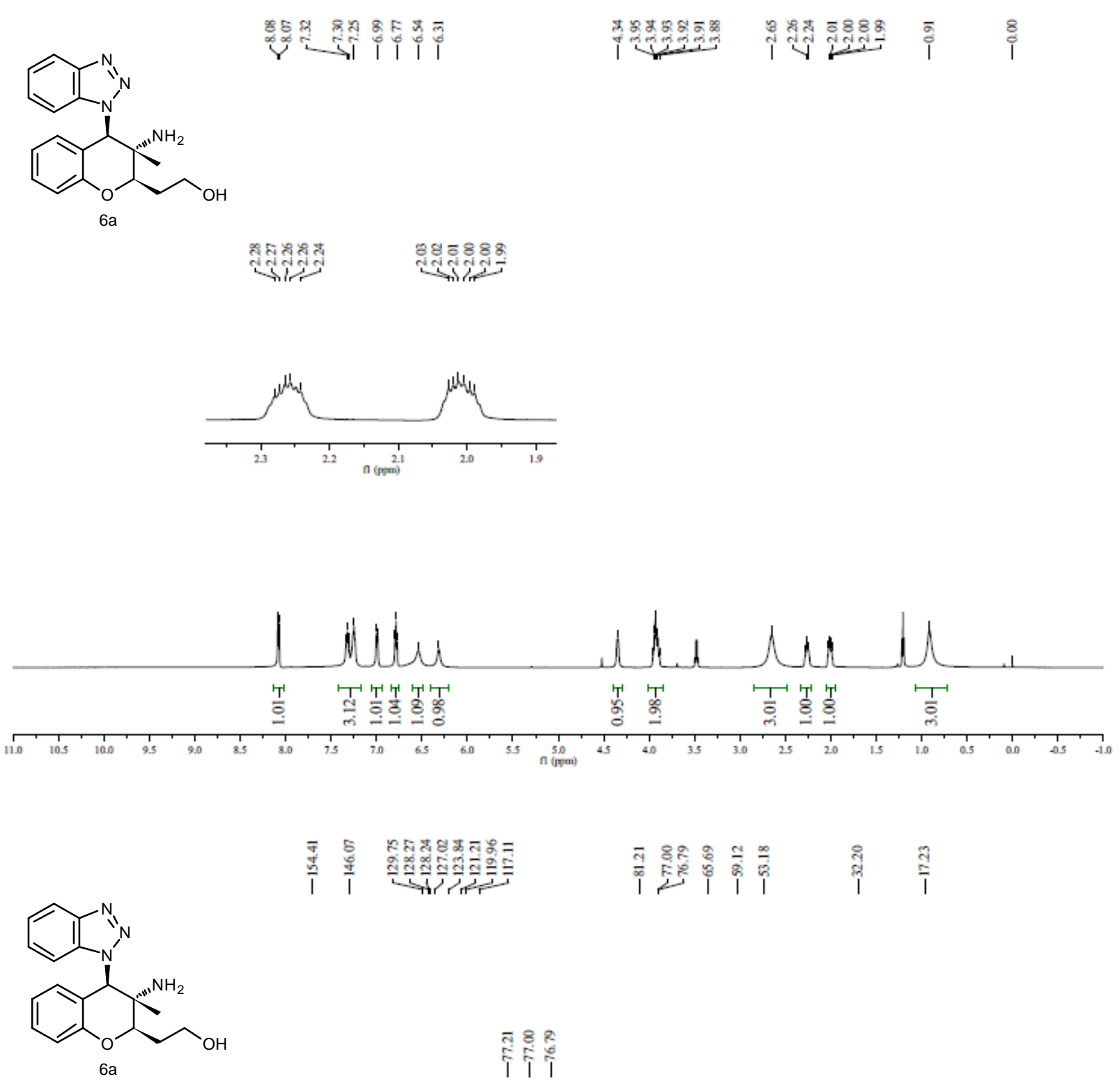

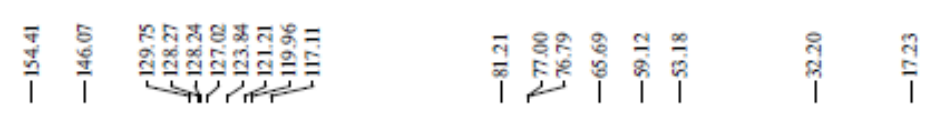

影影
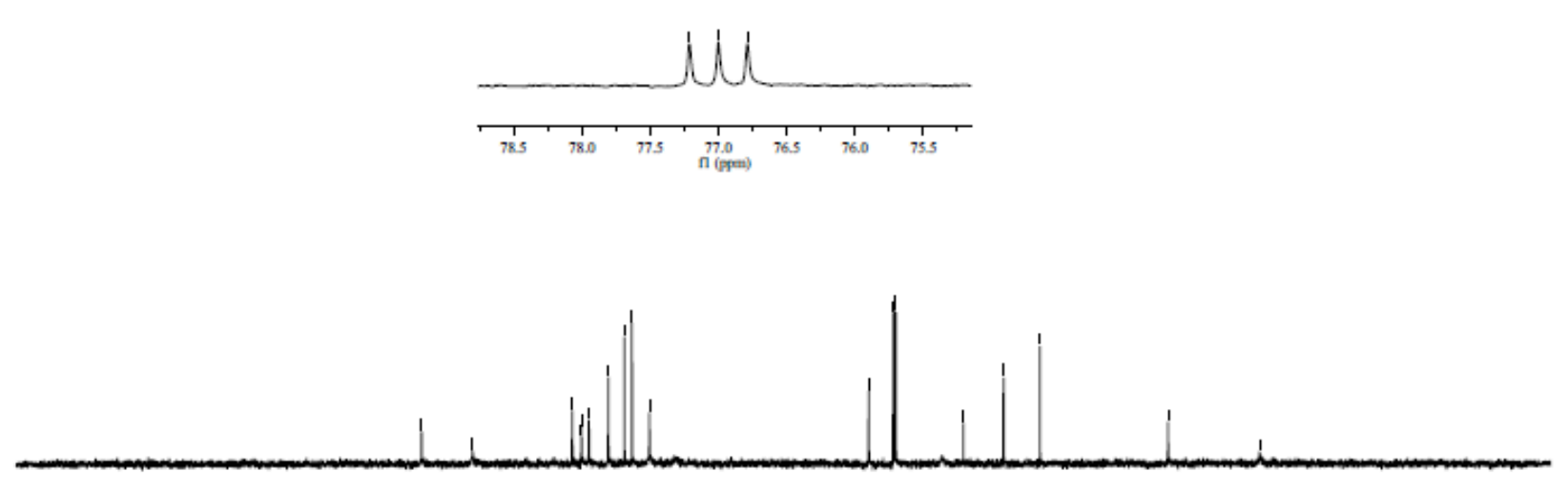

S-29 


\section{The copy of HPLC chromatograms}

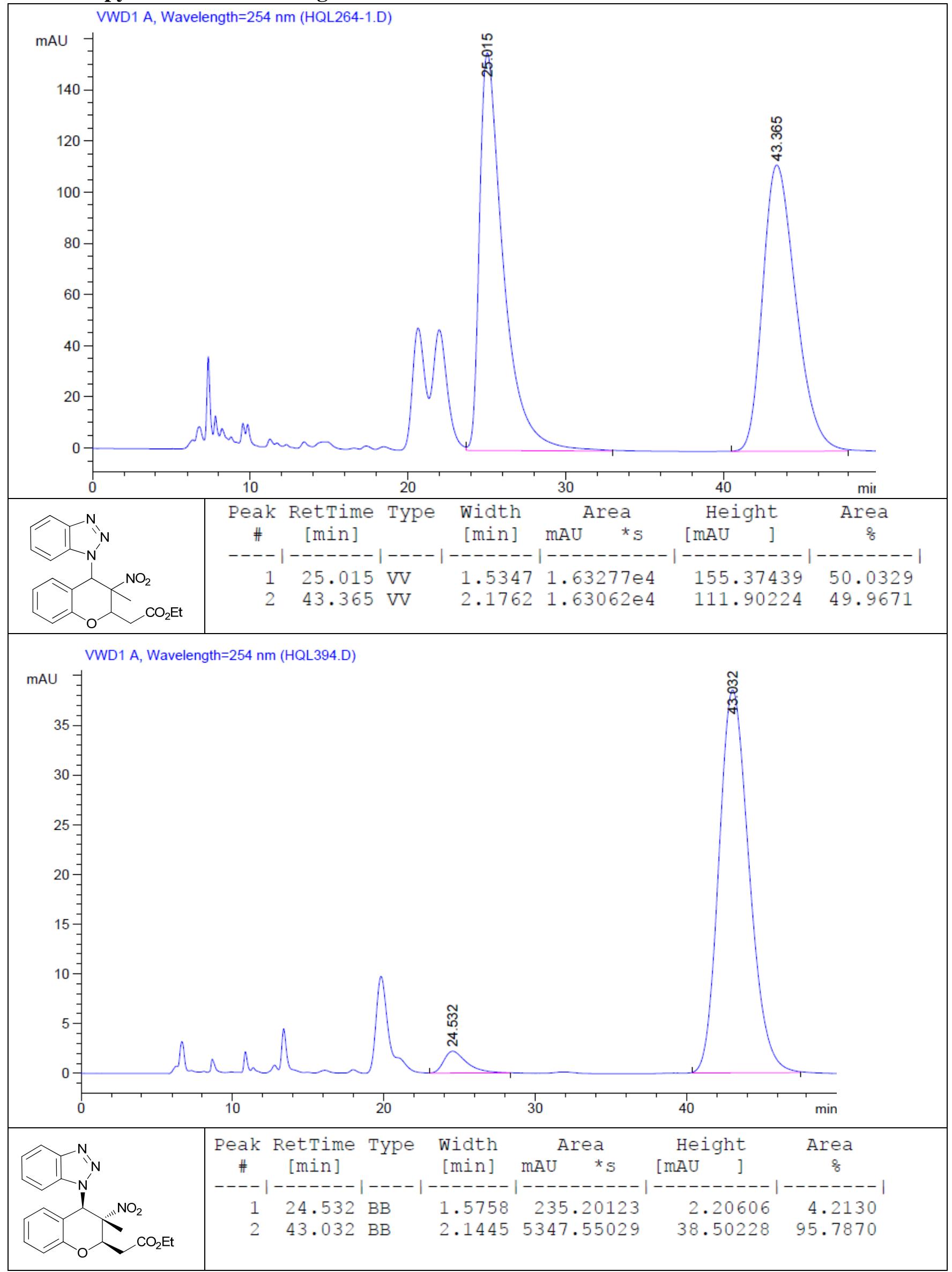




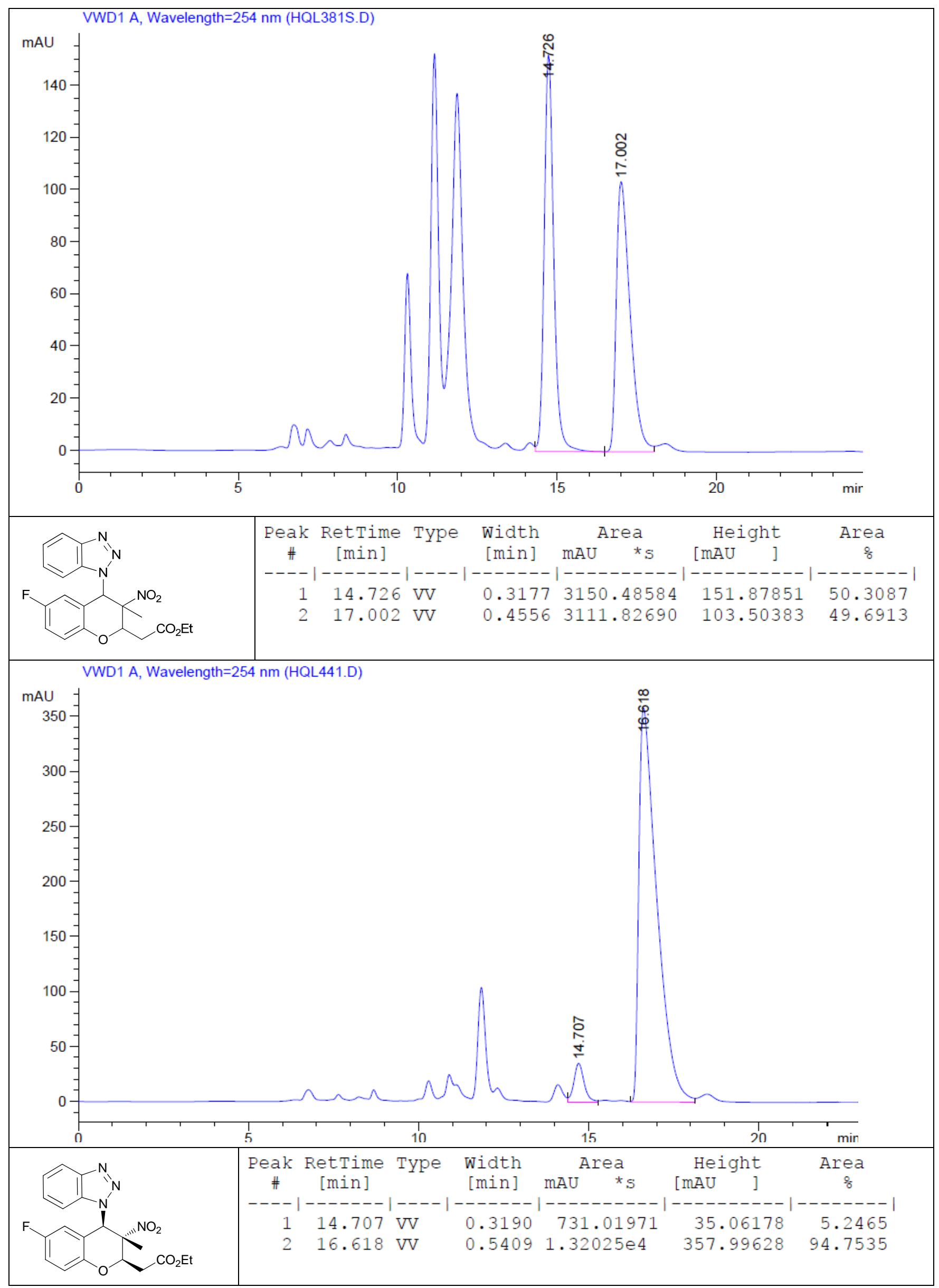




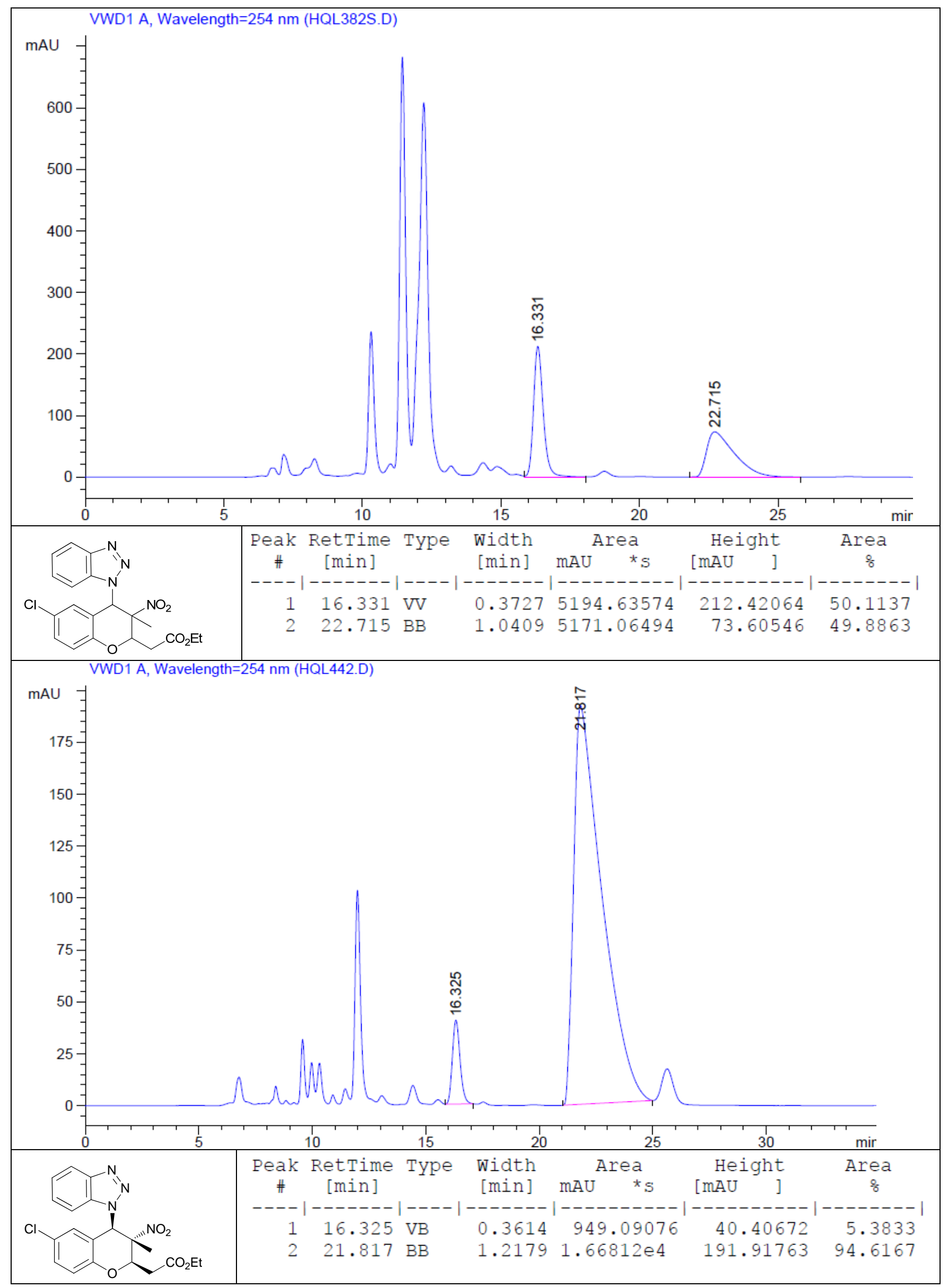




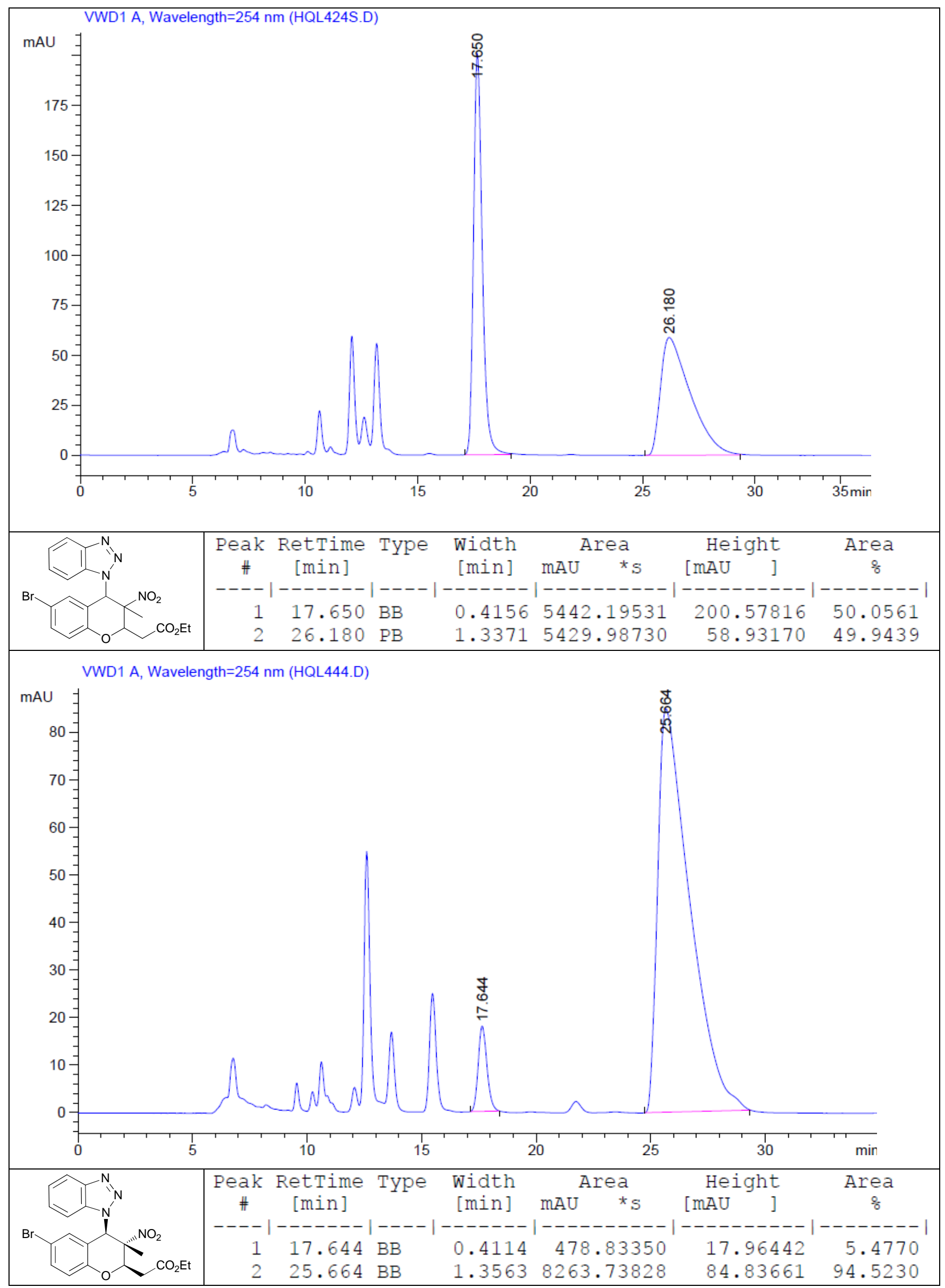




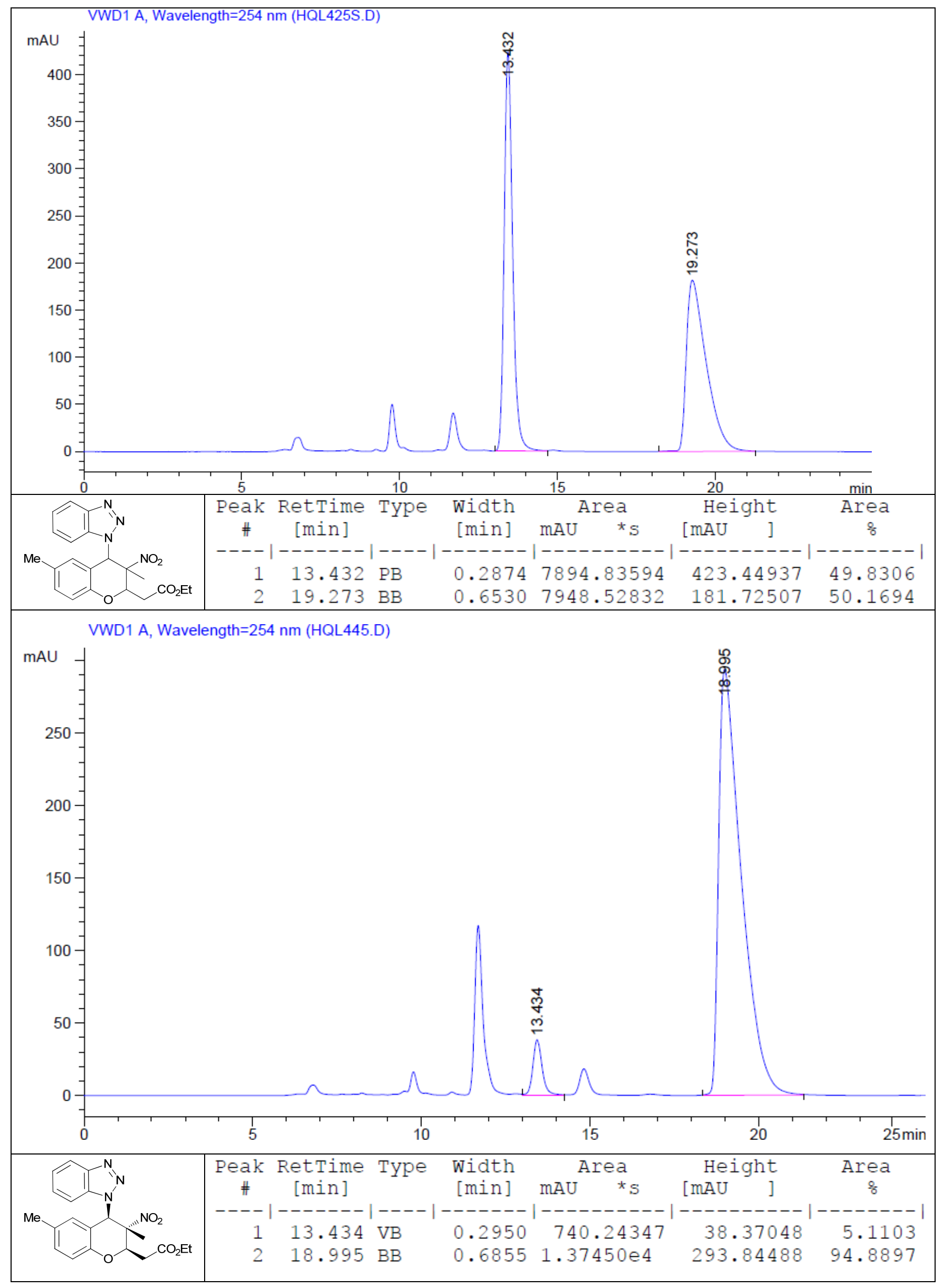




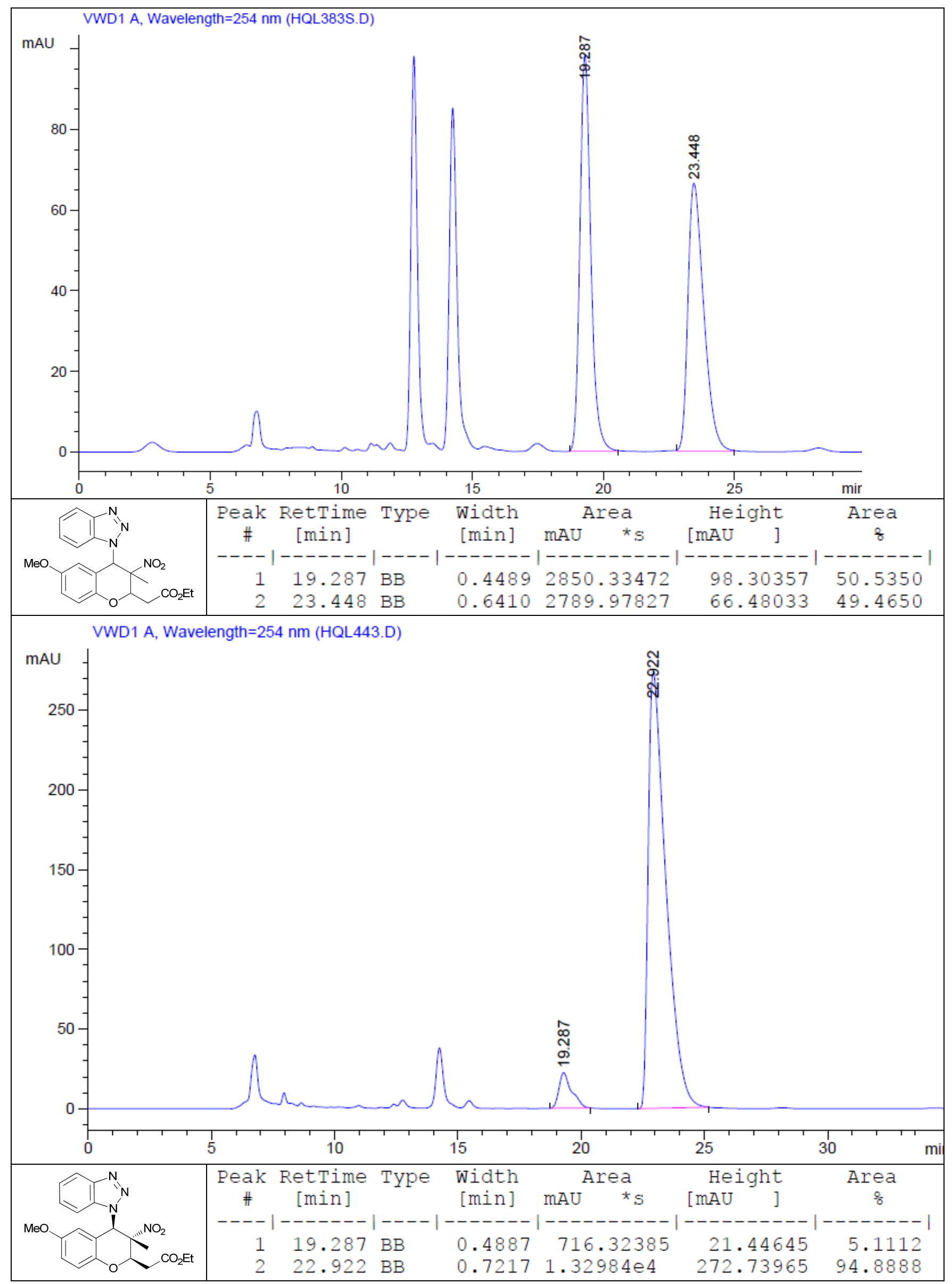




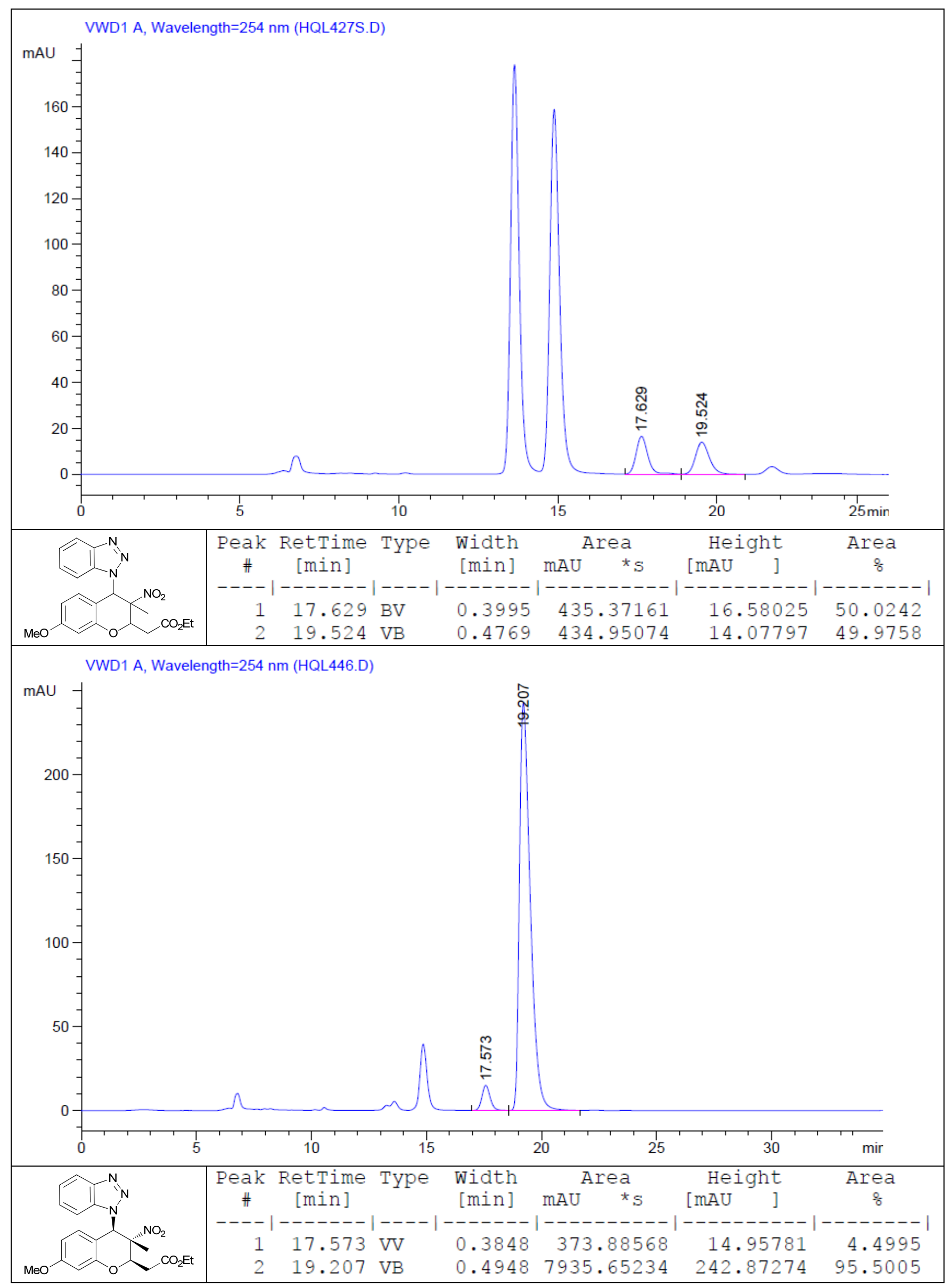




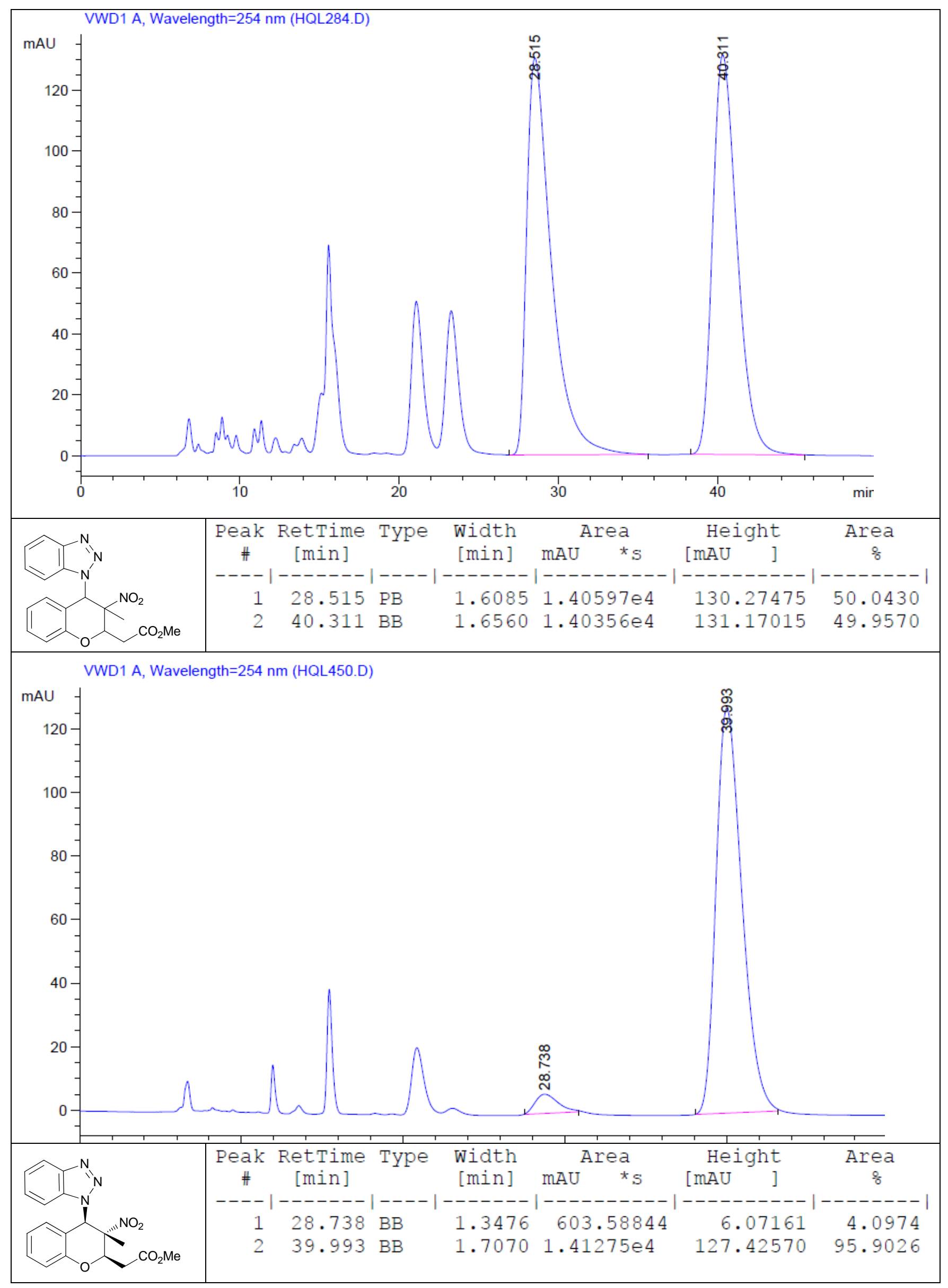




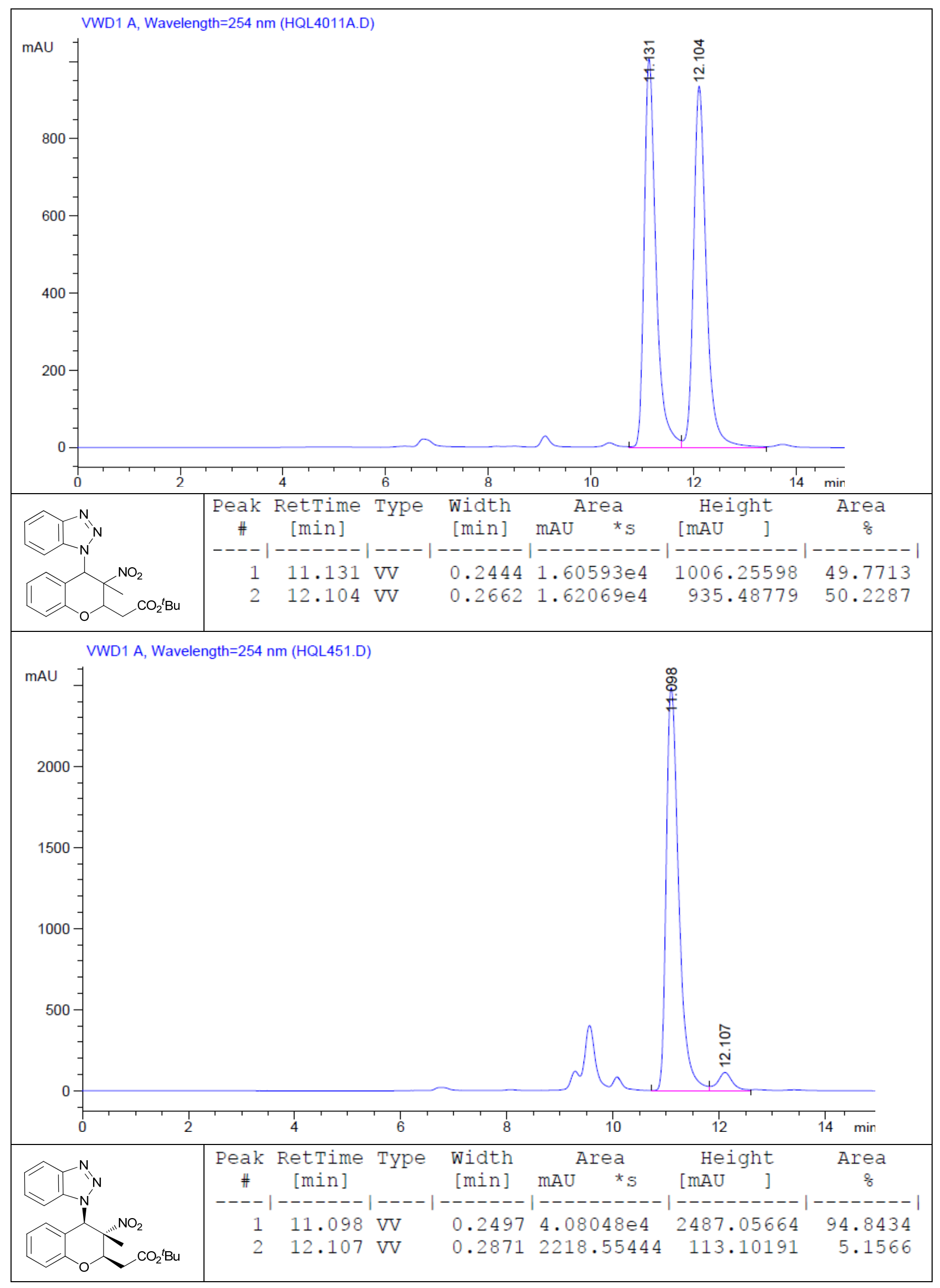




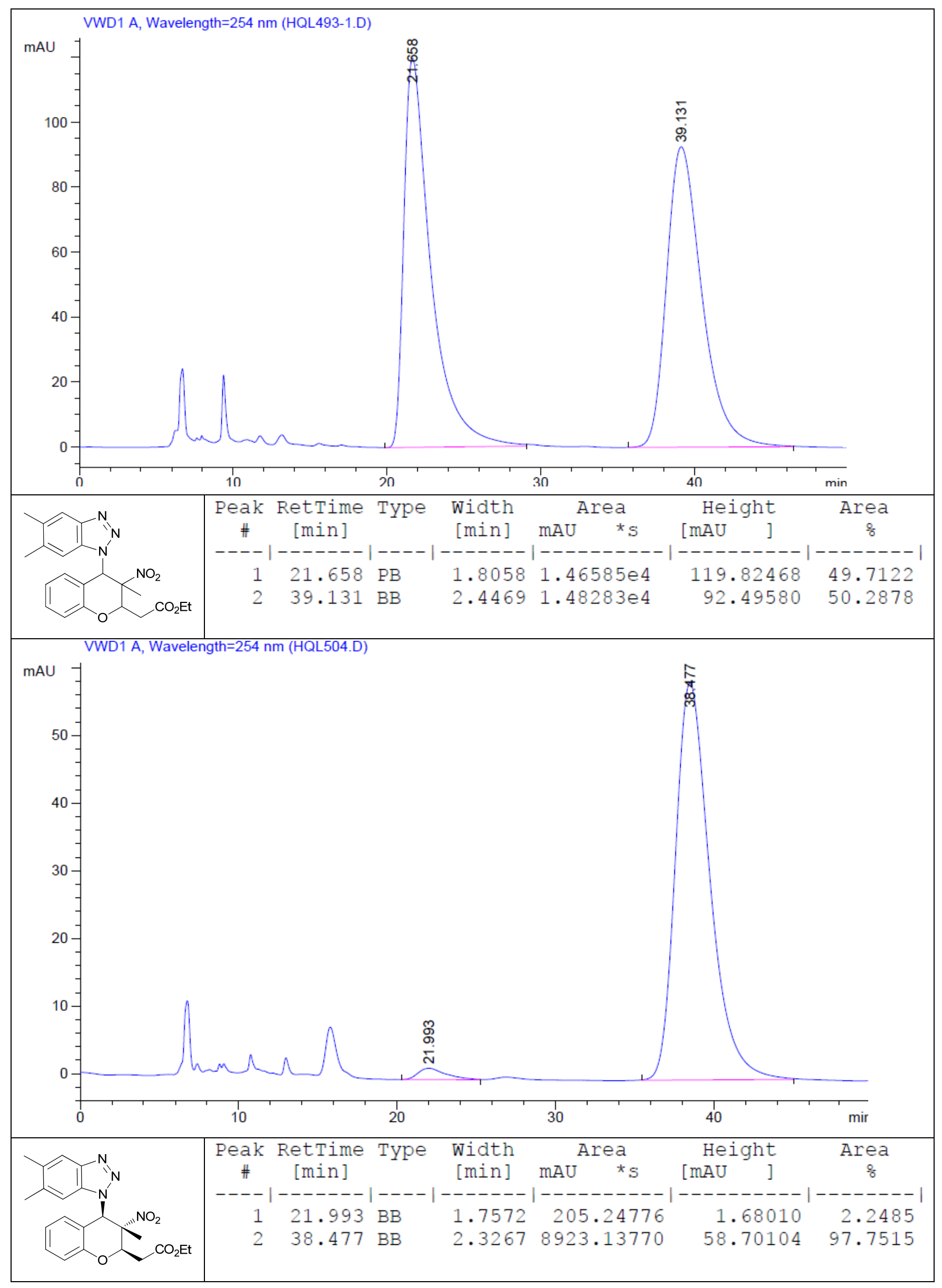




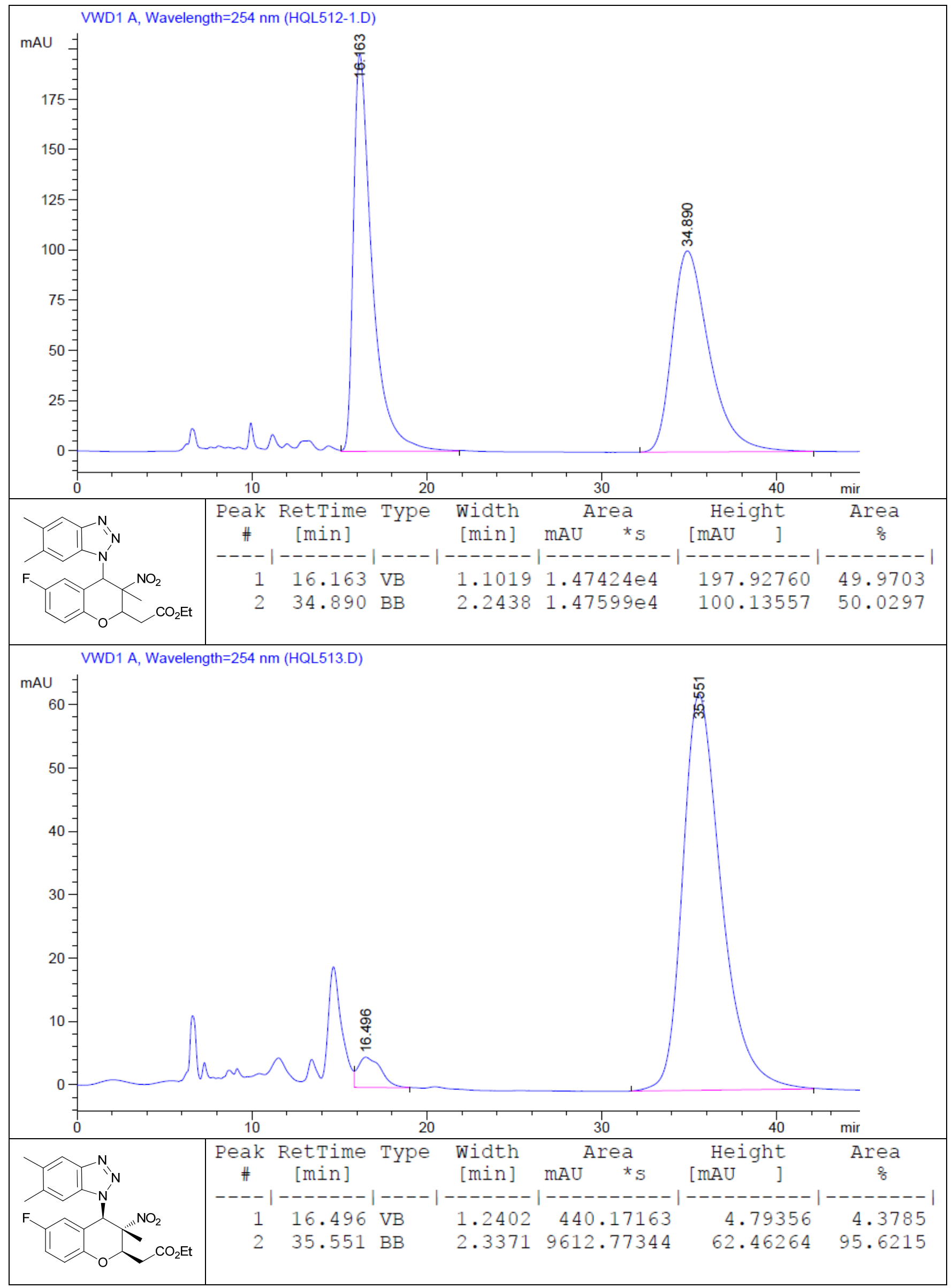




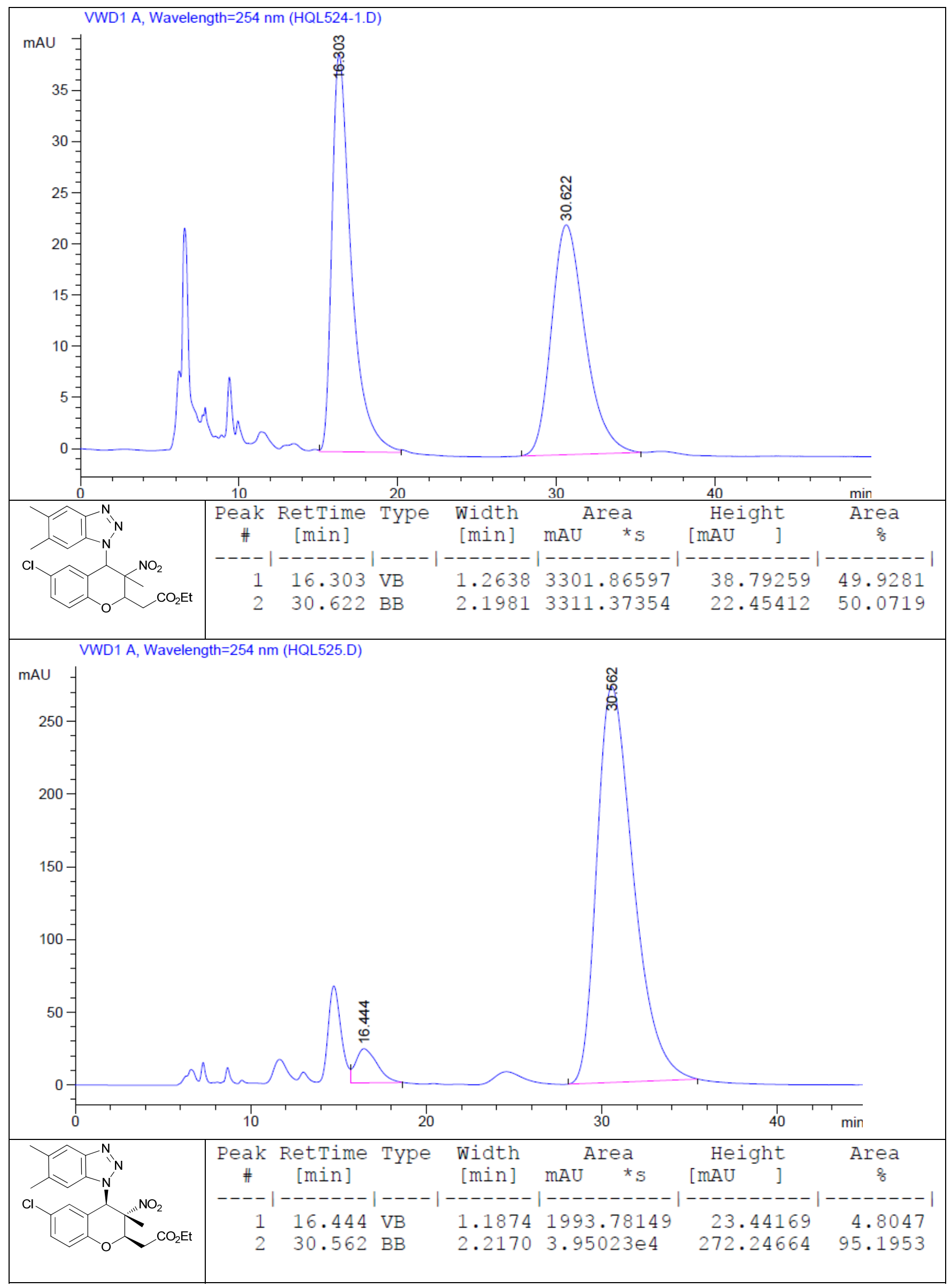




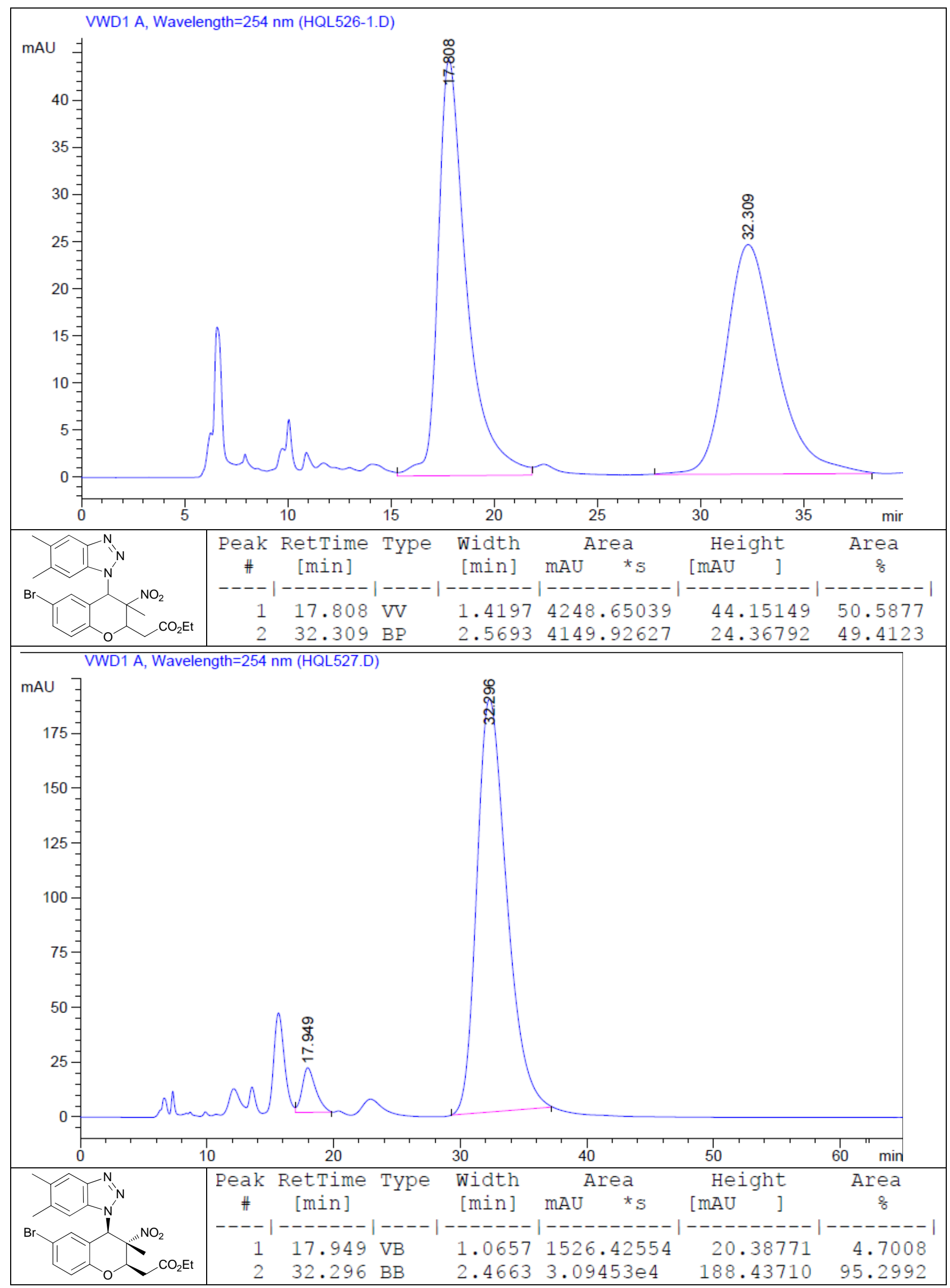




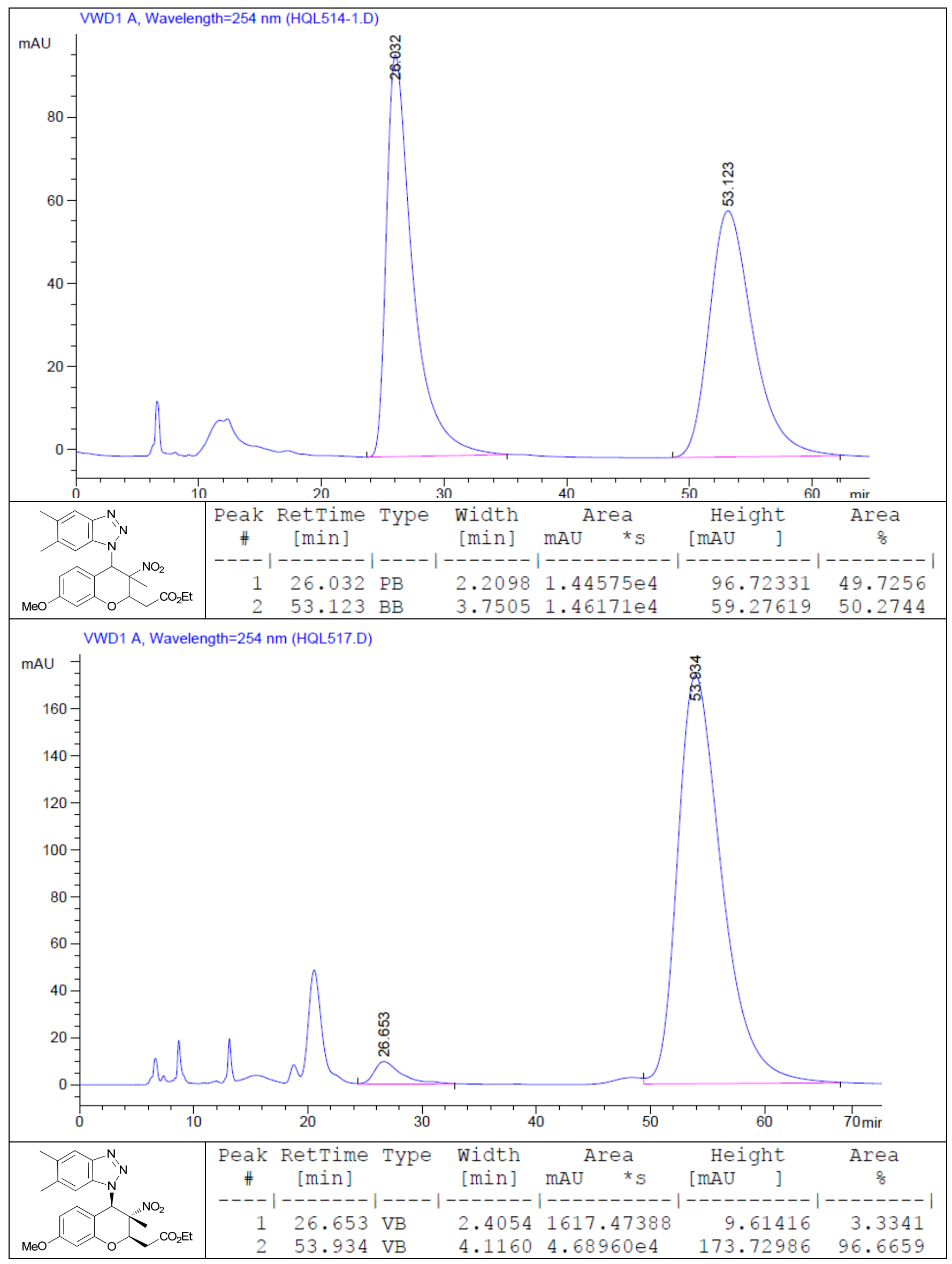




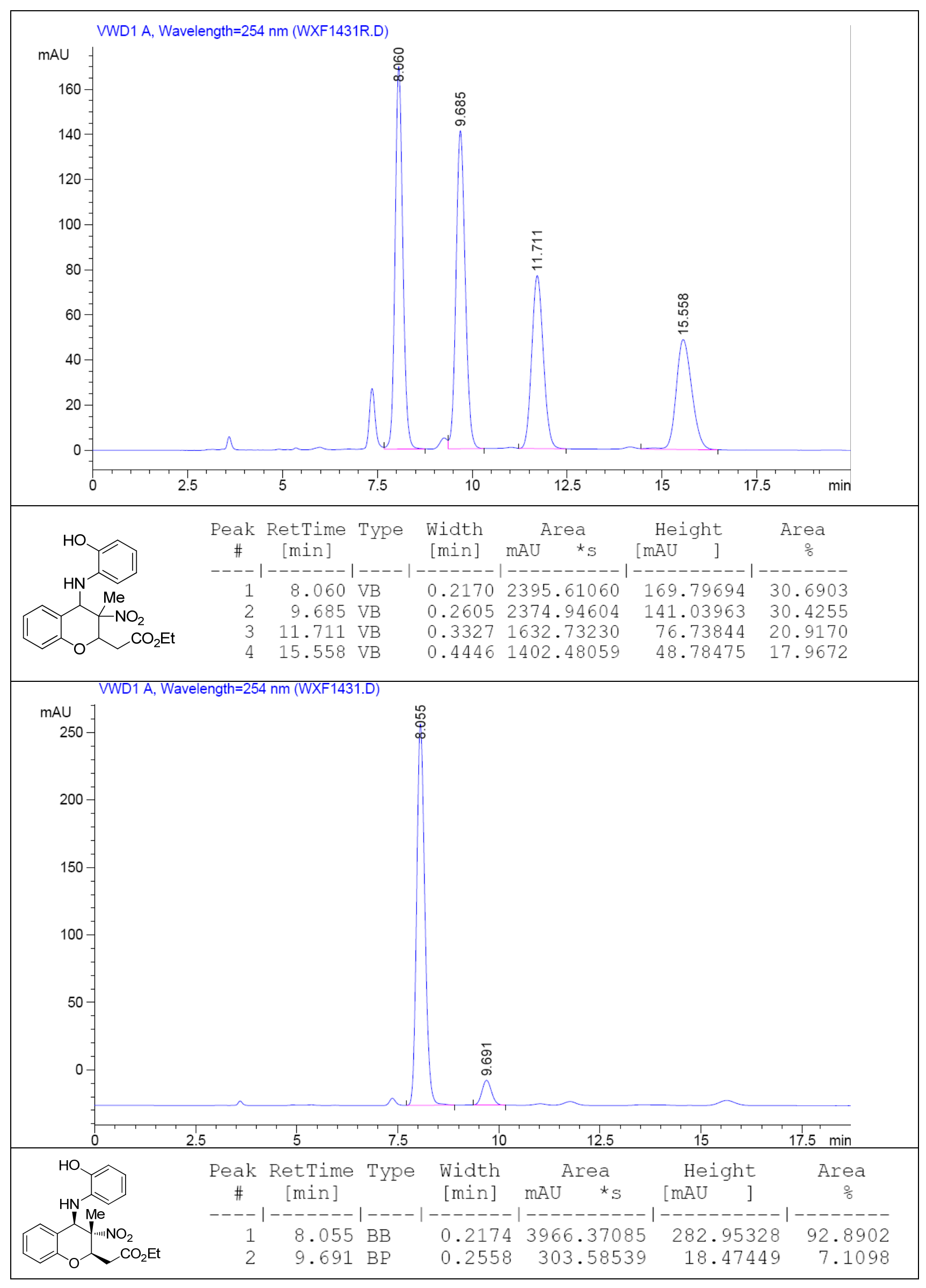

